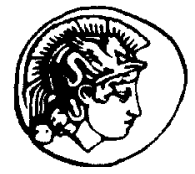

PERGAMON
Progress in Particle and Nuclear Physics 50 (2003) 63-152

\section{Progress in \\ Particle and \\ Nuclear Physics}

http://www.elsevier.com/locate/npe

\title{
Higgs Boson Theory and Phenomenology
}

\author{
M. CARENA ${ }^{1}$ and H.E. HABER ${ }^{2}$ \\ ${ }^{\prime}$ Fermi National Accelerator Laboratory, \\ P.O. Box 500, Batavia, IL 60510, USA \\ ${ }^{2}$ Santa Cruz Institute for Particle Physics, \\ University of California, Santa Cruz, CA 95064, USA
}

\begin{abstract}
Precision electroweak data presently favors a weakly-coupled Higgs sector as the mechanism responsible for electroweak symmetry breaking. Low-energy supersymmetry provides a natural framework for weakly-coupled elementary scalars. In this review, we summarize the theoretical properties of the Standard Model (SM) Higgs boson and the Higgs sector of the minimal supersymmetric extension of the Standard Model (MSSM). We then survey the phenomenology of the SM and MSSM Higgs bosons at the Tevatron, LHC and a future $e^{+} e^{-}$linear collider. We focis on the Higgs discovery potential of present and future colliders and stress the importance of precision measurements of Higgs boson propertics.
\end{abstract}

\section{Introduction--Origin of Electroweak Symmetry Breaking}

Deciphering the mechanism that breaks the electroweak symmetry and generates the masses of the known fundamental particles is one of the central challenges of particle physics. The Higgs mechanism [1] in its most general form can be used to explain the observed masses of the $W^{ \pm}$and $Z$ bosons as a consequence of three Goldstone bosons $\left(G^{ \pm}\right.$and $\left.G^{0}\right)$ that end up as the longitudinal components of the gauge bosons. These Goldstone bosons are generated by the underlying dynamics responsible for electroweak symmetry breaking. However, the fundamental nature of this dynamics is still unknown. Two broad classes of electroweak symmetry breaking mechanisms have been pursued theoretically. In one class of theories, electroweak symmetry breaking dynamics is weakly-coupled, while in the second class of theories the dynamics is strongly-coupled.

The electroweak symmetry breaking dynamics that is employed by the Standard Model posits a self-interacting complex doublet of scalar fields, which consists of four real degrees of freedom [2]. Renormalizable interactions are arranged in such a way that the neutral component of the scalar doublet arcquires a vacuum expectation value, $v=246 \mathrm{GeV}$, which sets the scale of electroweak symmetry 
breaking. Consequently, three massless Goldstone bosons are generated, while the fuurth saldar dente' of freedom that remains in the physical spectrum is the CP-even neutral Higgs boson (hsw) wf the Standard Model. It is further assumed in the Standard Model that the scalar doublet alsu inmplen (1) fermions through lukawa interactions. After electroweak srmmetry breaking, these intorctims : in responsible for the generation of quark and charged lepton masses. This approach is an cxanple of with electroweak symmetry breaking. Assuming that $m_{h_{s M}} \lesssim 200 \mathrm{GeV}$. all fields remain weakly interaring at energies up to the Planck scale. In thr weakly-ropled approach to electruwrak swmetry buraking. the Standard Model is very likely embedded in a supersymmetric theory $|3|$ in order to staliliz. llo large gap between the electroweak and the Planck scales in a natural way $[1,5]$. These theories proticy a spectrum of Higgs scalars [6], with the properties of the lightest Higgs scalar often msembliug than if the Standard Model (SM) Higgs boson.

Alternatively, strong breaking of electroweak symmetry is accomplished by new strong interactuns near the TeV scale [']]. More recently, so-called "little Higgs models" have been proposed in in hich the scale of the new strong interactions is pushed up abore $10 \mathrm{Tr} \mathrm{V}^{2}$ [8]: and the lightest Higge scalar resembles the weakly-coupled SM Higgs boson. In it more sperulative direction. it new appruach w. clectroweak symmetry breaking has been explored in which extra spate dimernsions beyond the muat $3+1$ dimensional spacetime are introduced [9] with characteristic sizes of order $(\mathrm{TeV}):$ J

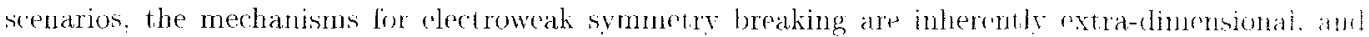
the resulting phenomenology may be significantly different from the usnal ajpluathes ment inated atwon.

Ill these alternative appruaches lie outside the scope of this reviow

Although there is as yet no direct evidence for the nature of electroweak symmetry braking drmanics, present data can be used to discriminate among the different approaches. For example, precision clectroweak data, accumulated in the past decade at LEP, SLC 6 the Tevatron and elsewhere, strongly support the Standard Model with a weakly-coupled Higgs boson [10]. Moreover, the contribution of

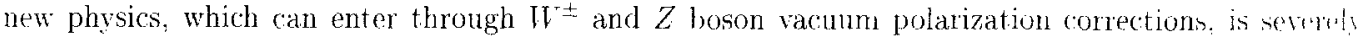
constrained. This fact has already served to rule out several models of strongly-coupled electrowerk symmetry breaking dynamics. The Higgs boson contributes to the $W^{ \pm}$and $Z$ boson vacmum polariziltion through loop effects, and so a global Standard Model fit to the electroweak data vields information about the Higgs mass. The results of the LEP Electroweak Working Group analysis shown in fig. $1 / a 1$ yield [10]: $m_{h_{S M}}=81_{-33}^{+52} \mathrm{GeV}$, and provides a $93 \%$ CL upper limit of $m_{h_{\text {s.M }}}<193 \mathrm{GeV}$. Thess sults reflect the logarithmic sensitivity to the Higgs mass via the virtual Higgs loop contributions 16; the various electroweak observables. The $95 \%$ CL upper limit is consistent with the direct searches at T.F.P [11] that show wo conclusive evidenes for the Higgs boson, and imply that mhan $>114.4$ Gel an 95\% CL. Fig. 1(b) exhibits the most probable range of values for the SM1 Higgs mass [12]. This nians range is consistent with a woakly-coupled Higgs scalar that is expected to emerge from the Standaral Model scalar dynamics (although the Standard Model does not predict the mass of the Higgs boson: rather it relates it to the strength of the scalar self-coupling).

There are some loopholes that can be exploited to circumvent this conclusion. It is possibse in 

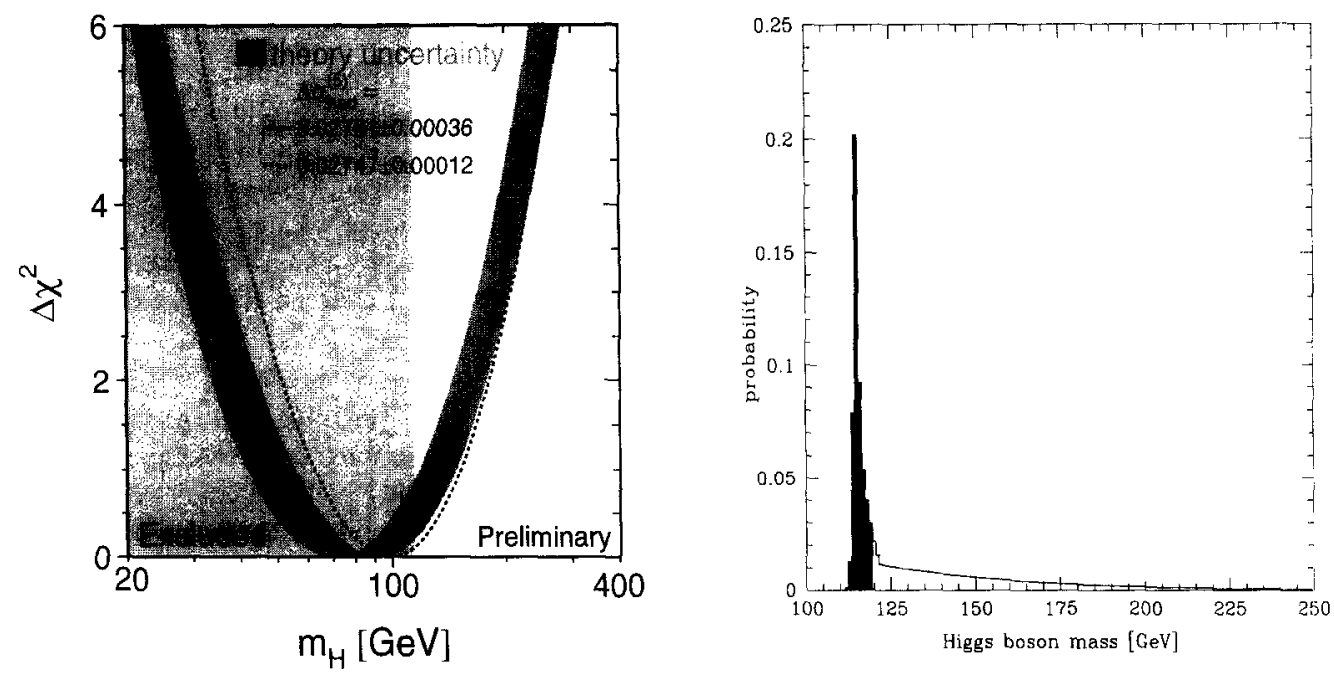

Figure 1: (a) The "blueband plot" shows $\Delta \chi^{2} \equiv \chi^{2}-\chi_{\min }^{2}$ as a function of the Standard Model Higgs mass [10]. The solid line is a result of a global fit using all data; the band represents the theoretical error due to missing higher order corrections. The rectangular shaded region shows the $95 \% \mathrm{CL}$ exclusion limit on the Higgs mass from direct searches at LEP [11]. (b) Probability distribution function for the Higgs boson mass, including all available direct and indirect data [12]. The probability is shown for $1 \mathrm{GeV}$ bins. The shaded and unshaded regions each correspond to an integrated probability of $50 \%$

construct models of new physics where the goodness of the global Standard Model fit to precision electroweak data is not compromised while the strong upper limit on the Higgs mass is relaxed. In particular, one can construct effective operators [13,14] or specific models [15] of new physics where the Higgs mass is significantly larger, but the new physics contributions to the $W^{ \pm}$and $Z$ vacuum polarizations, parameterized by the Peskin-Takenchi [16] parameters $S$ and $T$, are still consistent, with the experimental data. In addition, some have argued that the global Standard Model fit exhibits possible internal inconsistencies [17], which would suggest that systematic uncertainties have been underestimated and/or new physics beyond the Standard Model is required. Thus, although weaklycoupled electroweak symmetry breaking seems to be favored by the strong upper limit on the Higgs mass, one cannot definitively rule out all other approaches.

Nevertheless, one additional piece of data is very suggestive. Within the supersymmetric extension of the Standard Model, grand unification of the electromagnetic, the weak and the strong gauge interactions can be achieved in a consistent way, strongly supported by the prediction of the electroweak mixing 
angle at low energy scales with an accuracy at the percent level [18.19]. The significance of this prediction is not easily matched by other approaches. For example, in strongly-coupled electroweak symmetry breaking models, unification of couplings is not addressed per se. whoreas in cxtra-dimensional models it is often achieved by introducing new structures at intermediate euergy scales. Unless one is willing to regard the apparent gauge coupling unification as a coincidence. it is tempting to conclude that weak electroweak symmetry breaking with low-energy supersymmetry is the preferred mechanism, leading to an expected mass of the lightest Higgs boson below $200 \mathrm{GeV}$ (less than $135 \mathrm{GeV}$ in the simplest. supersymmetric models), and a possible spectrum of additional neutral and charged Higgs bosons with masses up to of order $1 \mathrm{TeV}$.

Henceforth, we shall assume that the dynamics of electroweak symmetry breaking is a result w a weakly-coupled scalar sector. The Standard Model is an effertive field theory and provides sin wy good description of the physics of elementary particles and their interactions at an energy scale of $\mathcal{O}(100) \mathrm{GeV}$ and below. However, there must exist somo energy scale, $\mathrm{A}$. at which the Standard Worke breaks down. That is, the Standard Model is no longer adequate for describing the theory abow 1 . and degrees of freerlom associaterl with new physics hecomo relevant. In particular. we know that $\Lambda \leq M_{\mathrm{PL}}$, since at an energy scale above the Planck scale, $M_{\mathrm{PL}_{\mathrm{L}}} \therefore 11^{14}$ (ie $\mathrm{V}$, quantum gravitatiunat rffects become significant and the Standard Model must be replared br a more fundamental thesm that

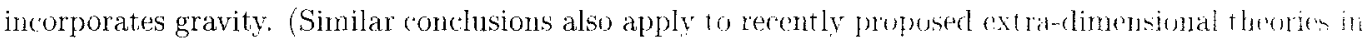
which quantum gravitational effects can become significant at energies scales is low as $\mathcal{O}(1$ Tel Of course. it is possible that new physics beyond the Standard Model exists at an energy scale between the electroweak and Planck scale, in which case the value of $A$ might lie significantly below $Y_{\mathrm{P}}$.

The value of the Higgs mass itself can provide an important constraint on the vahue of 1 . If $m_{1+\infty}$ in

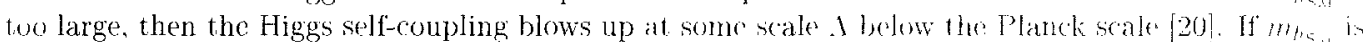

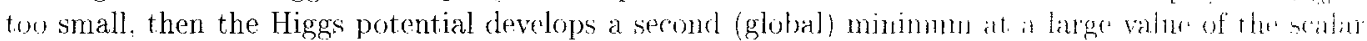
field of order $\Lambda$ [21]. Thus new physics must enter at a scale $\Lambda$ or below in urder that the global miniminu of the theory correspond to the observed $\mathrm{SU}(2) \times \mathrm{U}(1)$ broken vacuum with $:=246 \mathrm{GeV}$, Thus. wiven a value of $\Lambda$, one can compute the minimum and maxinum Higgs mass allower. The results of this computation (with shaded bands indicating the theorelical uncerlaing of the resthly) are illustrated in fig. 2(a) [22]. Consequently, a Higgs mass range $130 \mathrm{GeV} \lesssim m_{h_{t: 31}} \lesssim 180 \mathrm{G}(\mathrm{G}$ is consistent with m effective Standard Model that survives all the way to the Planck scale."

However, the survival of the Standard Model as an effective theory all the way up to the Planch scale is unlikely based on the following "naturalness" [1] argument. In an effective field theory particle

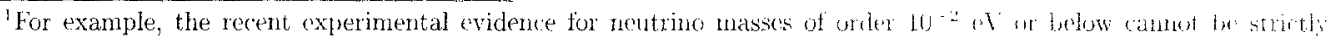
cxplained in the Standard Model. Yet one can easily write down a dimension-5 operator responsible for neutrimo massts that is suppressed by $n / \Lambda$. If $m_{i} \sim 10^{*-2}$ of then one obtains as a rough estimate $\Lambda \leqq 10^{17}$ Gol

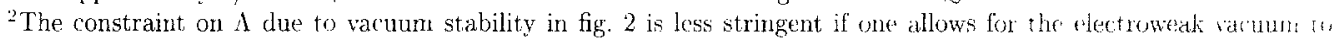
be metastable, with a lifetime greater than the age of the universe. An analysis of ref. [23] fincls that fur a sufficirnty

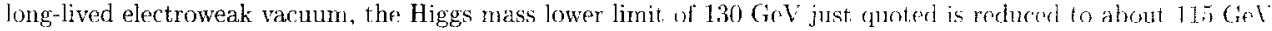



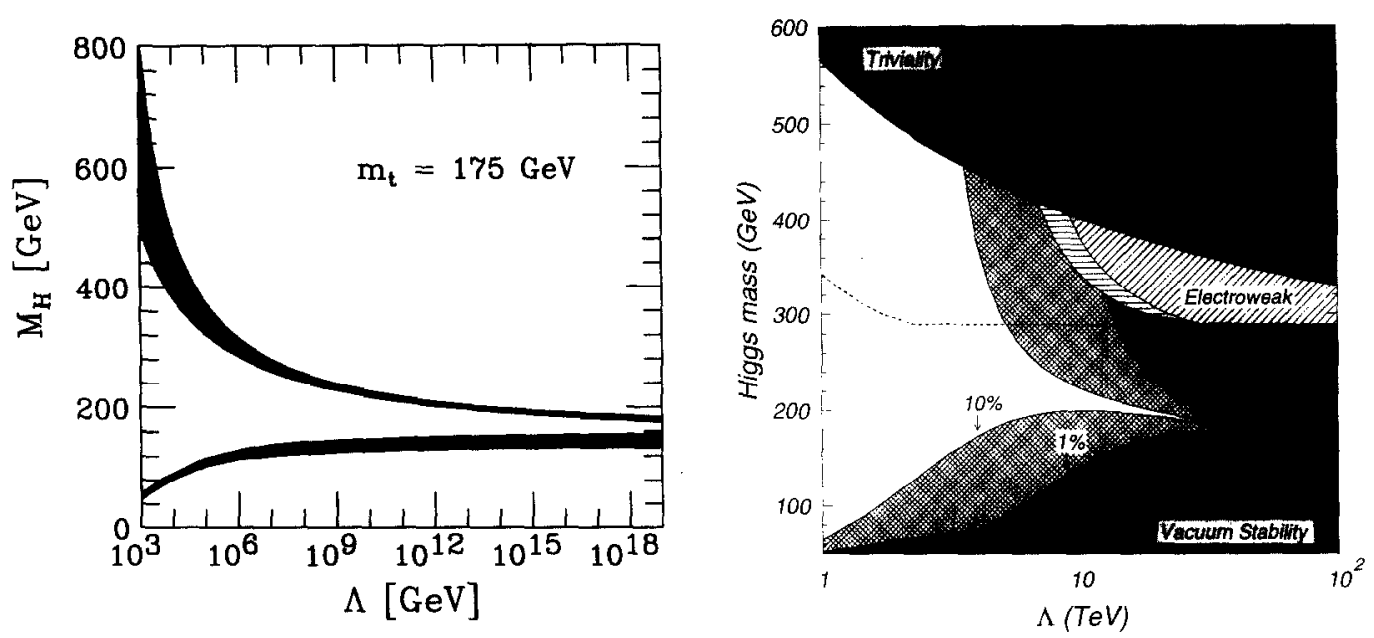

Figure 2: (a) The upper [20] and the lower [21] Higgs mass bounds as a function of the energy scale $\Lambda$ at which the Standard Model breaks down, assuming $M_{t}=175 \mathrm{GeV}$ and $\alpha_{s}\left(m_{Z}\right)=0.118$, taken from ref. [22]. The shaded areas above. reflect the theoretical uncertainties in the calculations of the Higgs mass bounds. (b) Following ref. [14], a reconsideration of the $\Lambda$ vs. Higgs mass plot with a focus on $\Lambda<100 \mathrm{TeV}$. Precision electroweak measurements restrict the parameter space to lie below the dashed line, based on a $95 \%$ CL fit that allows for nonzero values of $S$ and $T$ and the existence of higher dimensional operators suppressed by $v^{2} / \Lambda^{2}$. The unshaded area has less than one part in ten fine-tuning.

masses and dimensionless couplings of the low-energy theory are calculable in terms of parameters of a more fundamental theory that describes physics at the energy scale $\Lambda$. All low-energy couplings and fermion masses are logarithmically sensitive to $\Lambda$. In contrast, scalar squared-masses are quadratically sensitive to $\Lambda$. Thus, in this framework, the observed Higgs mass (at one-loop) has the following form:

$$
m_{h_{\mathrm{SM}}}^{2}=\left(m_{h}^{2}\right)_{0}+\frac{k g^{2} \Lambda^{2}}{16 \pi^{2}}
$$

where $\left(m_{h}\right)_{0}$ is a parameter of the fundamental theory, $g$ is an electroweak coupling and $k$ is a constant, presumably of $\mathcal{O}(1)$, that is calculable within the low-energy effective theory. Because these two contributions arise from independent sources, it is very unlikely that the magnitude of $m_{h_{S M}}^{2}$ is significantly smaller than either of the two terms. That is, the "natural" value for the physical scalar squared-mass is at least of order $g^{2} \Lambda^{2} / 16 \pi^{2}$. In order for this value to be consistent with the requirement that the Higgs mass is of order the electroweak symnetry breaking scale (as required from unilarity 
constraints $[24,25])$, the value of $\Lambda$ must satisfy

$$
\Lambda \simeq \frac{4 \pi m_{h_{S M}}}{g} \sim \mathcal{O}(1 \mathrm{TeV})
$$

If $\Lambda$ is significantly larger than $1 \mathrm{TeV}$ (often called the hierarchy problem in the literature), then the only way to generate a Higgs mass of $\mathcal{O}\left(m_{z}\right)$ is to have an "unnatural" cancellation between the two terms of eq. (1). This seems highly unlikely given that the two terms of eq. (1) have completely different origins. The requirement of $\Lambda \sim \mathcal{O}\left(1 \mathrm{TeV}^{\prime}\right)$ as a condition for the absence of fine-tuning of the Higgs mass parameter is nicely illustrated in fig. 2(b), taken from ref. [1]]

A viable theoretical framework that incorporates weakly-coupled Higgs bosons and satisfies the constraint of eq. (2) is that of "low-energy" or "weak-scale" supersymmetry [3] In this framework. supersymmetry is used to relate fermion and boson masses and interactions. Since ferrrion massin are only logarithmically sensitive to $A$. boson masses will exhibit the same logarithmic sensitivity if supersymmetry is exact. Since no supersymmetric partners of the Standard Model particles have betul found, it follows that supersymmetry is not an exact symmetry of the fundamental particle interactions. Hence, in the framework of low-energy supersymmetry. $\Lambda$ should be identified with the energy scale if supersymmetry-breaking. The naturalness constraint of eq. (2) is still relevant, which implies that the scale of supersymmetry breaking should not be much larger than a few TeV in order that the naturalnestes of scalar masses be preserved. Moreover, low-energy supersymmetry with a supersymmetry-breaking scale of $\mathcal{O}(1 \mathrm{TeV})$ is precisely what is needed to explain the obscrved gauge coupling unification in previously noted. We conclude that a suitable replacement for the Standard Model is a supersymmetric extension of the Standard Model as the effective field theory of the "TeV scale. One good feature of the supersymmetric approach is that the effective low-energy supersymmetric theory can be valid all the way up to the Planck scale, while still being natural!

The physics of the Higgs bosons will be explored by experiments now underway at the upgradid proton-antiproton Tevatron collider at Fermilab and in the near future at the Large Hadron Collidet (LHC) at CERN. Once evidence for electroweak symmetry breaking dynamic's is obtained. a more c'mln plete understanding of the mechanism involved will recuire experimentation at a future $c^{t}$ linear collider (LC) now under development. In this roview we focus primarily on the theory and phenomenology of the Standard Model Higgs buson and the Higgs bosons of low-energy supersymmetry. In Section? we review the theoretical properties of the Standard Model Higgs boson. and exhibit its main branching ratio and production rates at hadron colliders and at the LC. The main Higgs boson search technicunes at the Tevatron, LHC and the LC are described. In Section 3, we examine the Higgs bosons of the minimal supersymmetric Standard Model (MSSM). We summarize the tree-level properties of the MSSM Higgs sector and describe the most significant effects of the radiative corrections to the computation of the Higgs masses and couplings. We then exhibit the main branching ratios and production rates of the MSSM Higgs bosons and survey the phenomenology of the MSSM Higgs sector at the Tevatron, LHC and LC. A brief summary concludes this review in Section 4. 


\section{The Standard Model Higgs Boson}

In the Standard Model, the Higgs mass is given by: $m_{h_{S M}}^{2}=\frac{1}{2} \lambda v^{2}$, where $\lambda$ is the Higgs self-coupling parameter. Since $\lambda$ is unknown at present, the value of the Standard Model Higgs mass is not predicted. However, other theoretical considerations, discussed in Section 1, place constraints on the Higgs mass as exhibited in fig. 2. In contrast, the Higgs couplings to fermions [bosons] are predicted by the theory to be proportional to the corresponding particle masses [squared-masses]. In particular, the SM Higgs boson is a CP-even scalar, and its couplings to gauge bosons, Higgs bosons and fermions are given by: ${ }^{3}$

$$
\begin{array}{ll}
g_{h f \bar{f}}=\frac{m_{f}}{v}, & g_{h V V}=\frac{2 m_{\mathrm{l}}^{2}}{v}, \quad g_{h h V V}=\frac{2 m_{V}^{2}}{v^{2}}, \\
g_{h h h}=\frac{3}{2} \lambda v=\frac{3 m_{h_{S M}}^{2}}{v}, & g_{h h h h}=\frac{3}{2} \lambda=\frac{3 m_{h_{S M}}^{2}}{v^{2}},
\end{array}
$$

where $h \equiv h_{\mathrm{SM}}, V=W$ or $Z$ and $v=2 m_{W} / g=246 \mathrm{GeV}$. In Higgs production and decay processes, the dominant mechanisms involve the coupling of the Higgs boson to the $W^{ \pm}, Z$ and/or the third generation quarks and leptons. Note that a $h_{\mathrm{SM}} g g$ coupling ( $g=$ gluon) is induced by virtue of a one-loop graph in which the Higgs boson couples to a virtual $t \bar{t}$ pair. Likewise, a $h_{\mathrm{SM}} \gamma \gamma$ coupling is generated, although in this case the one-loop graph in which the Higgs boson couples to a virtual $W^{+} W^{-}$pair is the dominant contribution. Further details of the SM Higgs boson properties are given in ref. [2]. A review of the SM Higgs properties and its phenomenology, with an emphasis on the impact of loop corrections to the Higgs decay rates and cross-sections can be found in ref. [26].

\subsection{Standard Model Higgs Boson Decay Modes}

The branching ratios for the main decay modes of a SM Iliggs boson are shown as a function of Higgs boson mass in fig. 3 and $4(a)$, based on the results obtained using the HDECAY program [27]. For Higgs boson masses below $135 \mathrm{GeV}$, the decay $h_{\mathrm{SM}} \rightarrow b \bar{b}$ dominates, whereas above $135 \mathrm{GeV}$, the dominant decay mode is $h_{\mathrm{SM}} \rightarrow W W^{(*)}$ (below $W^{+} W^{-}$threshold, one of the $W$ bosons is virtual as indicated by the star). Above $t \bar{t}$ threshold, the branching ratio into top-quark pairs increases rapidly as a function of Higgs mass, reaching a maximum of about $20 \%$ at $m_{h_{\mathrm{SM}}} \sim 450 \mathrm{GcV}$. The total Higgs width is obtained by summing all the Higgs partial widths and is displayed as a function of Higgs mass in fig. 4(b).

The leading effects of the QCD corrections to the Higgs decay to quark pairs $[28]^{4}$ can be taken into account by using the tree-level formula for the Higgs partial width (which depends on the quark

\footnotetext{
${ }^{3}$ The corresponding Feynman rules are obtained by multiplying the Higgs boson $-V V$ couplings by $i g^{\mu \nu}$ and the other Higgs couplings of eqs. (3) and (4) by a factor of $-i$. The appropriate combinatorial factors have been included.

${ }^{4}$ The formulae for the leading order QCD-corrections to $\Gamma\left(h_{\mathrm{SM}} \rightarrow q \tilde{q}\right)$ are nicely summarized in ref. [29]. The leading electroweak radiative corrections have been obtained in ref. [30]. A useful summary of results, which includes new two-loop contributions to the radiatively corrected $h_{\mathrm{SM}} \rightarrow b \bar{b}$ partial width, can be found in ref. [31].
} 


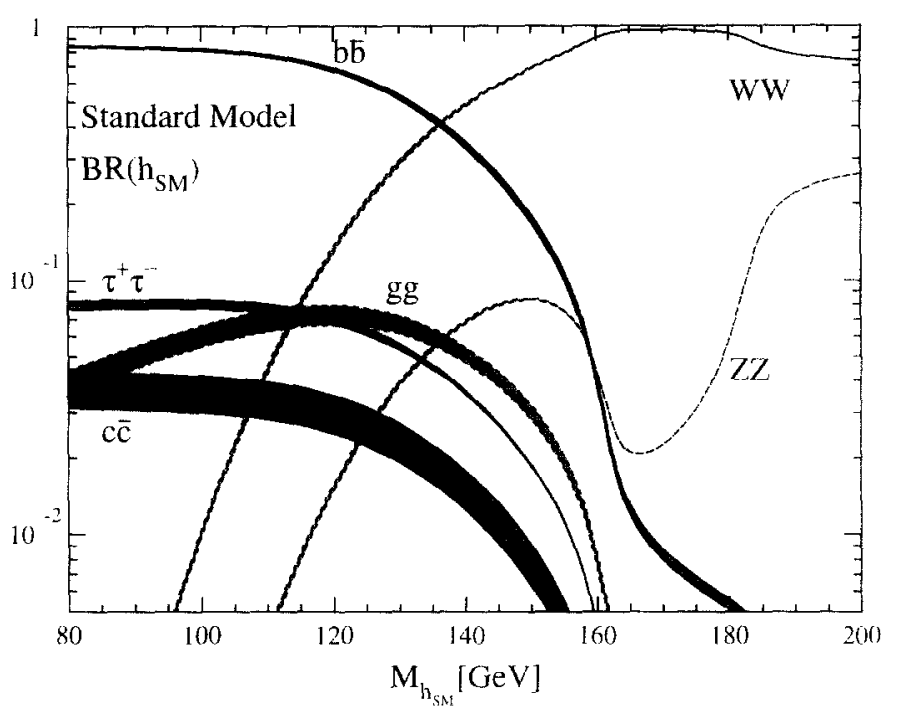

Figure 3: Branching ratios of the dominant decay modes of the Standard Model Higgs boson as a function of Higgs mass for $m_{h_{5 M}} \leq 200 \mathrm{GeV}$, taken from ref. [32]. These results have been obtained with the prograin HDECAY [27], and include QCD corrections beyond the leading order [29]. The shaded bands represent the rariations due to the micertainties in the: input parameters: $\alpha_{s}\left(M_{Z}^{2}\right)=0.120 \pm 0.003, \bar{m}_{b}\left(M_{b}\right)=4.22 \pm 0.05 \mathrm{GeV}, \bar{m}_{c}\left(M_{i}\right)=1.22 \pm 0.06 \mathrm{GeV}$, and $M_{t}=174 \pm \overline{G(t)}$.

mass), and identifying the quark mass with the running quark mass evaluated at the Higgs mass, $\bar{m}_{Q}\left(m_{h_{\mathrm{SM}}}\right)$. The running quark mass, $\bar{m}_{Q}\left(m_{h_{\mathrm{SM}}}\right)$ is obtained from the $\overline{\mathrm{MS}}$ mass, $\bar{m}_{Q}\left(M_{Q}\right)$ [where $M_{Q}$ is the corresponding quark pole mass], by renormalization group cvolution. The $\overline{\mathrm{MS}}$ quark masses ar' obtained from fits to experimental data [33]. Note that the large decrease in the charm quark mass due to QCD running is responsible for suppressing $\mathrm{BR}(c \bar{c})$ relative to $\mathrm{BR}\left(\tau^{+} \tau^{-}\right)$, in spite of the color enhancement of the former, thereby reversing the naively expected hierarchy. Below the corresponding two-body thresholds, the $W W^{(*)}, Z Z^{(*)}$ and $t^{(*)} \bar{t}$ decay modes (where the asterisk indicates an off-shell particle) are still relevant as shown in fig. 4 .

The $h_{\mathrm{SM}} g g, h_{\mathrm{SM}} \gamma \gamma$ and $h_{\mathrm{SM}} z_{\gamma}$ vertices are generated at one-loop. The partial width for $h_{S \mathrm{M}} \rightarrow g g \mathrm{i}$ is primarily of interest because it determines the $g g \rightarrow h_{\mathrm{S}, \mathrm{W}}$ production cross-section. The $h_{\mathrm{SM}}$, vert.ex is especially relevant both for the $h_{\mathrm{SM}} \rightarrow \gamma \gamma$ discovery mode at the LHC and for the $i ? \rightarrow h_{\mathrm{SM}}$ production mode at the LC operating as a $\gamma \gamma$ collider. 

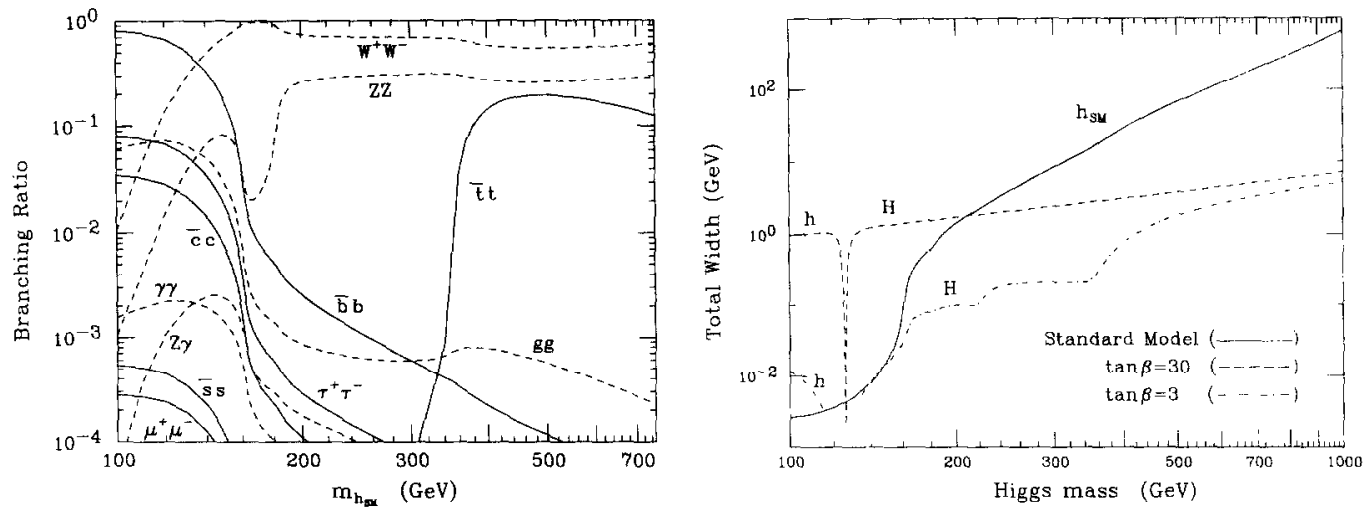

Figure 4: (a) Branching ratios of the Standard Model Higgs boson as a function of Higgs mass. Two-bosion [fermionantifermion] final states are exhibited by solid [dashed] lines. As compared with fig. 3, a larger range of Higgs masses and branching ratios are shown. (b) The total width of the Standard Model Higgs boson is shown as a function of its mass. For comparison, we exhibit the widths of the two CP-even scalars, $h$ and $H$ of the MSSM for two different choices of MSSM parameters ( $\tan \beta=3$ and 30 in the maximal mixing scenario; the onset of the $H \rightarrow h h$ and $H \rightarrow t \bar{t}$ thresholds in the $\tan \beta=3$ curve are clearly evident). The central values of $\alpha_{s}, \bar{n}_{b}\left(M_{b}\right)$ and $\bar{m}_{c}\left(M_{c}\right)$ quoted in the caption of fig. 3 are employed in both (a) and (b).

\subsection{Standard Model Higgs Boson Production at Hadron Colliders}

\subsubsection{Cross-sections at hadron colliders}

This section describes the most important Higgs production processes at the Tevatron $(\sqrt{s}=2 \mathrm{TeV})$ and the LHC $(\sqrt{s}=14 \mathrm{TcV})$. The relevant cross-sections are exhibited in figs. 5 and 6 [32,34 36]. Combining these Higgs production mechanisms with the decays discussed in Section 2.1, one obtains the most promising signatures.

Due to the large luminosity of gluons at high energy hadron colliders, $g g \rightarrow h_{\mathrm{SM}}$ is the Higgs production mechanism with the largest cross-section at the Tevatron and the LHC $[37,38]$. The twoloup. next to-leading order (NLO) QCD corrections enhance the gluon fusion cross-section by about a factor of two $[38,39]$. The corresponding NLO differential cross-section (as a function of the Higgs boson $\nu_{T}$ and rapidity) has also been obtained [40]. Recently, the next-to-NLO (NNLO) QCD corrections have been evaluated [41], and show a further enhancement of about 10\% to $30 \%$ depending on the Higgs mass and center-of-mass energy of the collider. The remaining scale dependence and the effects of higher order terms not yet computed are estimated to give a theoretical uncertainty of 10-20\%. The 
dependence of the gluon fusion cross-section on different parton densities vields roughly an additional nincertainty of order $10 \%$.

The cross-section for $q \ddot{q} \rightarrow W^{ \pm} h_{\mathrm{SM}}$ (summed over both $W$ charge states) is the second largest Higgs cross-section at the Tevatron for $m_{h_{S M}} \lesssim 175 \mathrm{GeV}$. At the LHC, the $W^{+} h_{\text {su }}$ cross-section in not as prominent over the Higgs mass range of interest. The corresponding $4 \bar{q}>Z h_{5 M}$ cross-section is roughly a factor of two lower than the corresponding $W^{ \pm} h_{\mathrm{S} N}$ eross-section. The QCD corrections to $\sigma\left(q \bar{q} \rightarrow V h_{\mathrm{SM}}\right)[V=W$ or $Z]$ coincide with those of the Drell-Yan process and increase the ressisections by about $30 \%[42-44]$. The theoretical uncertainty is stimated to be about $15 \%$ from the remaining scale dependence. The dependence on different sets of parton densities is ratber weak and also leads to a variation of the production cross-sertions by about $15 \%$

vector boson fusion is a shorthand notation for the full $q \dot{q} \rightarrow q q h_{\text {sin }}$ proes'ss: where the quark ark anti-quark both radiate virtual vector bosons $\left(\mathrm{I}^{*}\right)$ which then annihilate to produce the Higgs bosirn. Vector boson fusion via $u d \rightarrow d u h_{S M}$ (and its charge-conjugate process) is also porssible. In figs 3 and 16 . all contributing processes are included, and the sum of all such contributions is labeled $q q \rightarrow q q q_{h}$ in for simplicity. The QCD corrections enhance the cross-section by abont 10\%. [4.45]. The vector busen fusion process is the second largest Higgs cross-section at the LHC: its cross-section approaches the $g g \rightarrow h_{\mathrm{SM}}$ cross-section for $m_{h_{s M}} \sim 1 \mathrm{TeV}$.

The cross-sections for $g g, q \ddot{q} \rightarrow t t h_{\mathrm{SM}}$ at the Tevatron and the LHC are displayed in tigs 5 and $t$. The NLO QCD corrections to $q \bar{q} \rightarrow t \bar{t} h_{\mathrm{SM}}$ have recently been computed in refs. [46] and $[47]$. and the corrections to $g g \rightarrow t \bar{t} h_{\mathrm{SM}}$ have heen ohtained in rof. [47]. Fig. 5 includes the complete NLO QC'D

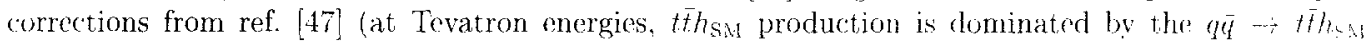
subprocess). The size of the QCD corrections depends sensitively on the choice of srale. H. cruplewer in the running coupling constant and parton distribution functions. Changes in $\mu$ can signiticaisty modify the tree-level cross-sections, whereas the NLO-corrected cross-sections are rather insensitiw in reasonable changes in $\mu$. In fig. 6, the tree-level th of the partonic center-of-mass energy), and is roughly a factor of two smaller than the corresponding rross-section with $\mu=m_{t}$. With respect to the latter choice. the NLO-rorrections of ref. [47] are rather small, typically of order 10-20\% depending on the precise choice of $/ 1$.

The tree-level $g g, q \bar{q} \rightarrow b \bar{b} h_{S M}$ cross-section (as a function of $m_{h_{\mathrm{SM}}}$ ) shown in fig. $\bar{j}$ has been computed by fixing the scales of the parton distribution functions. the cumning coupling $o_{s}$ and the running Higgs -bottom-quark Yukawa coupling (or equivalently, the cunning $b$-quark mass) at the walue of the corresponding $h_{5 \mathrm{M}}$ mass. In fig. 6 , the $b$-quark pole mass is employed, ${ }^{5}$ while the scales for $r$. and the parton distribution functions were set equal to the partonic center-of-mass energy.

By using the running $b$ quark mass, one implicitly resums large logarithms associated with the (OCDcorrected Yukawa coupling. Thus, the tree-level $b \bar{b} h_{\mathrm{SM}}$ cross-section displayed in fig. 5 implicitly includes

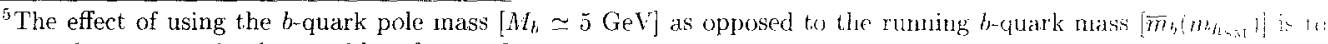
increase the cross-section by roughly a factor of two.
} 


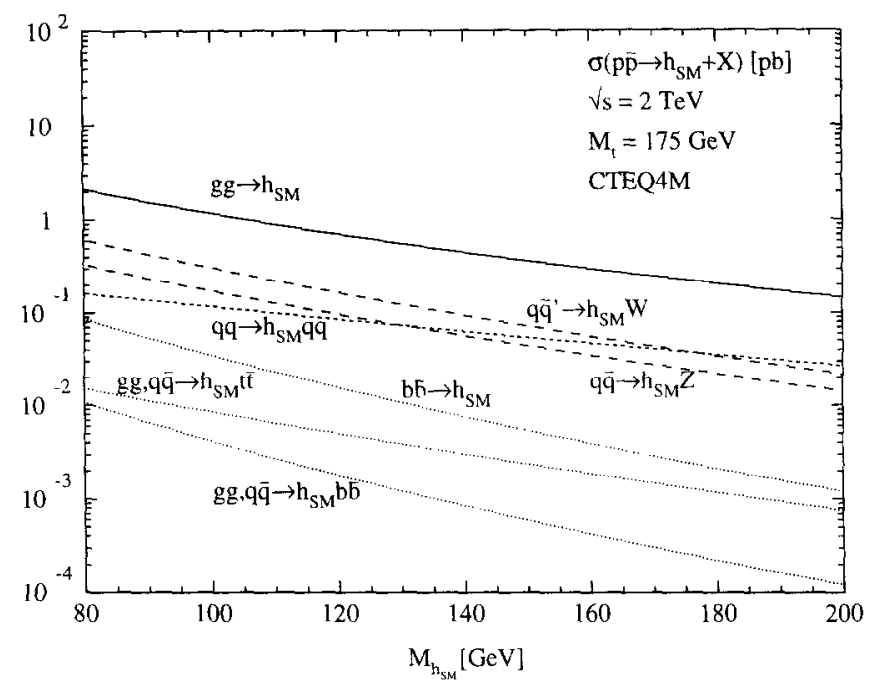

Figure 5: Higgs production cross-sections (in units of $\mathrm{pb}$ ) at the Tevatron $[\sqrt{s}=2 \mathrm{TeV}]$, for the various production mechanisms as a function of the Higgs mass, taken primarily from refs. [32] and [35]. The full NLO QCD-corrected results are employed for the gluon fusion $g g \rightarrow h_{\mathrm{SM}}$, vector boson fusion $q q \rightarrow V^{*} V^{*} q q \rightarrow h_{\mathrm{SM}} q q$ (here, $q q$ refers to both $u d$ and $q \bar{q}$ scattering), Higgs-strahlung processes $q \bar{q} \rightarrow V^{*} \rightarrow V h_{\mathrm{SM}}$ (where $V=W^{ \pm}, Z$ ), $b \bar{b} \rightarrow h_{\mathrm{SM}}$ (taken from ref. [36]), and $g g, q \bar{q} \rightarrow h_{\mathrm{SM}} t \bar{t}$. Tree-level cross-sections are exhibited for $g g, q \bar{q} \rightarrow h_{\mathrm{SM}} b \bar{b}$. In the latter case, the cross-section has been computed with a running $b$-quark mass, $\alpha_{s}$ and the parton distribution functions all evaluated at the corresponding Higgs mass.

a part of the QCD corrections to the full inclusive cross-section. However, the most significant effect of the QCD corrections to $b \bar{b} h_{\mathrm{SM}}$ production arises from the kinematical region where the $b$ quarks are emitted near the forward direction. In fact, large logarithms arising in this region spoil the convergence of the QCD perturbation series since $\alpha_{s} \ln \left(m_{h_{\mathrm{SM}}}^{2} / m_{b}^{2}\right) \sim \mathcal{O}(1)$. These large logarithms (already present at lowest order) must be resummed to all orders, and this resummation is accomplished by the generation of the $b$-quark distribution function [48,49]. Thus, the QCD-corrected fully inclusive $b \bar{b} h_{\mathrm{SM}}$ cross-section can be approximated by $b \bar{b} \rightarrow h_{\mathrm{SM}}$ and its QCD corrections. ${ }^{6}$ The latter is also exhibited in fig. 5 and is

\footnotetext{
${ }^{6}$ This result, although correct in the far asymptotic regime (where $\sqrt{s} \gg m_{h_{\mathrm{SM}}} \gg m_{b}$ ), may still not be reliable for Higgs production at the Tevatron and LHC. In ref. [50], it is argued that even at the LHC for $m_{h_{S M}}=500 \mathrm{GeV}$, sizable $m_{h}$-effects still remain and $\sigma\left(b \bar{b} \rightarrow h_{S M}\right)$ is an overestimate of the true QCD-corrected $b \bar{b} h_{S M}$ cross-section.
} 


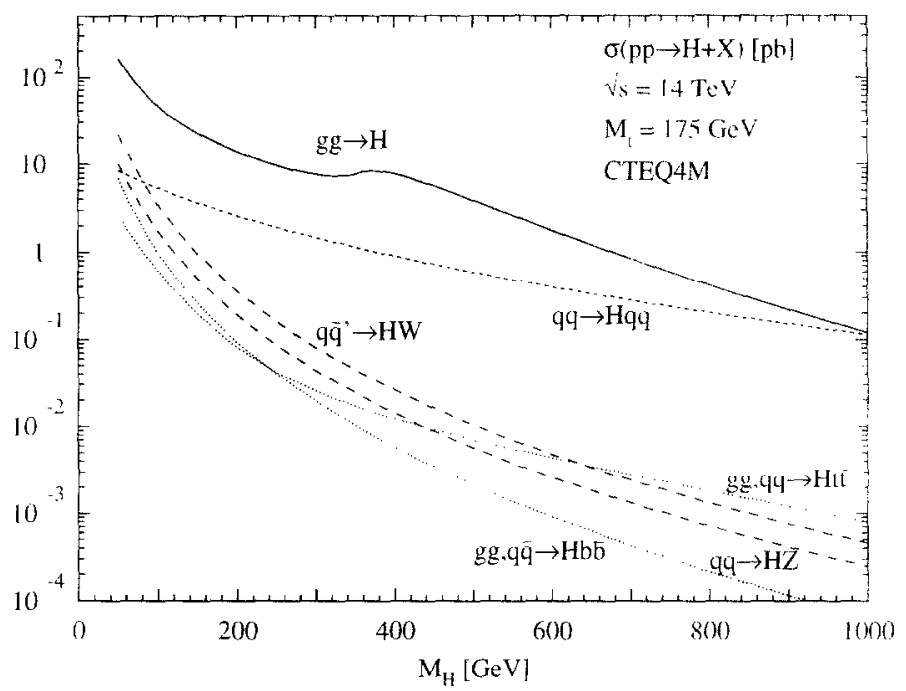

Figure 6: Higgs production cross-sections (in units of $\mathrm{pb}$ ) at the $\mathrm{LHC}[\sqrt{s}=14 \mathrm{TeV}]$. for the various production mechanisms as a function of the Higgs mass, taken from ref. [44]. The cross-section curves for $g g, q \bar{q} \rightarrow h_{\mathrm{SM}} t \bar{t}$ (which has not been updated to include the NLO calculation of ref. [47]) and $g g, q \bar{q} \rightarrow h_{\mathrm{SM}} b \bar{b}$ are based on a tree-level calculation with $t$-quark and $b$-quark pole masses and $\alpha_{s}$ and the parton distribution functions evaluated at the partoric center-of-mass energy.

seen to be roughly an order of magnitude larger than the tree-level $6 b / 4 \mathrm{n}$ cross-hection 36$]$. (of rourse. this result is not very relevant for the searches at hadron colliders in which transverse momentum cuts on the $b$-jets are employed. Ultimately, one needs the QCD-corrected differential cross-section for $b \bar{b} h_{\mathrm{SW}}$ (as a function of the final state $b$-quark transverse momentum) in order to do realistic simulations of the Higgs signal in this channel. However, if only one $b$-quark jet is tagged, it may be sutticient to consider the process $b g \rightarrow b h_{\mathrm{SM}}$. The cross-section for $b g \rightarrow b h_{\mathrm{SM}}$ at lowest order can be found in ref. [51]: the NLO QCD-corrected cross-section has been recently obtained in ref. [52]. For example, assuming that: $p_{T^{\prime}}>15 \mathrm{GeV}$, and the pseudorapidity $|\eta|<2$ for the observed $b$-quark jet. the NLO cross-section at the Tevatron ranges from about $6 \mathrm{fb}$ to $0.25 \mathrm{fb}$ for $100 \mathrm{GeV} \leq m_{h_{\mathrm{SM}}} \leq 200 \mathrm{GeV}$. Increasing the ants $1, \mathrm{c}$ $p_{T}>30 \mathrm{GeV}$ and $|\eta|<2.5$ at the LHC yields a range of cross-sections from about $200 \mathrm{fb}$ to $1.2 \mathrm{tb}$ tor $100 \mathrm{GeV} \leq m_{h_{\mathrm{SM}}} \leq 500 \mathrm{GeV}$.

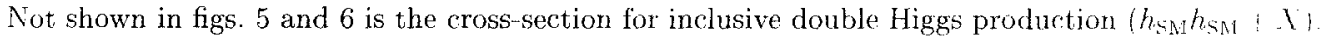


Double Higgs production is not observable at the Tevatron, but may be possible to detect at the LHC given sufficient luminosity. The main contributions to double Higgs production in order of importance are: (i) $g g \rightarrow h_{\mathrm{SM}} h_{\mathrm{SM}}$; (ii) $V V \rightarrow h_{\mathrm{SM}} h_{\mathrm{SM}}$; and (iii) $q \vec{q} \rightarrow V h_{\mathrm{SM}} h_{\mathrm{SM}}$, where $V=W$ or $Z$. The gluon-gluon fusion cross-section dominates by at least an order of magnitude, so we focus on this subprocess [53 56]. Including NLO QCD corrections, typical cross-sections for $p p \rightarrow h_{\mathrm{SM}} h_{\mathrm{SM}}+X$ at $\sqrt{s}=14 \mathrm{TeV}$ range from about $40 \mathrm{fb}$ to $10 \mathrm{fb}$ for $100<m_{h_{\mathrm{SM}}}<200 \mathrm{GeV}[55,56]$. There are two classes of diagrams that contribute to $h_{\mathrm{SM}} h_{\mathrm{SM}}$ production via gluon fusion: $g g \rightarrow h_{\mathrm{SM}}^{*} \rightarrow h_{\mathrm{SM}} h_{\mathrm{SM}}$ (via the top-quark triangle diagram) which is sensitive to the triple-Higgs vertex, and $g g \rightarrow t^{*} t^{*} \rightarrow h_{\mathrm{SM}} h_{\mathrm{SM}}$ (via the top-quark box diagram) which is independent of the triple-Higgs vertex. Due to the relatively low cross-sections, it will be very challenging to extract information on the Higgs self-coupling parameter from LHC data.

Finally, assuming that very forward protons can be tagged, diffractive production of Higgs bosons may provide a viable signal at the LHC (the corresponding cross-sections at the Tevatron are probably too small) $[57,58] .{ }^{7}$ Such events are characterized by rapidity gaps, i.e., the absence of particle production between the forward protons and the centrally produced Higgs boson. In particular, for $m_{h_{\mathrm{SM}}} \sim 130 \mathrm{GeV}$, the exclusive process $p p \rightarrow p p h_{\mathrm{SM}}$ (with $h_{\mathrm{SM}} \rightarrow b \bar{b}$ ) can be used at the LHC to obtain the Higgs mass with an accuracy of about $1 \%$ by measuring the invariant mass recoiling against the final state protons [59].

\subsubsection{Standard Model Higgs Boson Searches at the Tevatron}

In the mass region of interest to the Tevatron Higgs search (100 GeV $\left.\lesssim m_{h_{\mathrm{SM}}} \lesssim 200 \mathrm{GeV}\right)$, the SM Higgs boson is produced most copiously via $g g$ fusion, with a cross-section from about $1.0-\cdots-1$ pb. For $m_{h_{\mathrm{SM}}} \lesssim 135 \mathrm{GeV}$, the Higgs boson decays dominantly to $b \bar{b}$. Since the cross-section for the QCD production of $b \bar{b}$ dijet events is orders of magnitude larger than the Higgs production cross-section, the $g g \rightarrow h_{S M} \rightarrow b \bar{b}$ channel is not a promising channel. For $m_{h_{S M}} \gtrsim 135 \mathrm{GeV}$, the Higgs boson decays dominantly to $W W^{(*)}$ (where $W^{*}$ is a virtual $W$ ), and the channel $g g \rightarrow h_{\mathrm{SM}} \rightarrow W^{(*)}$ is accessible to the Tevatron Higgs search [60].

Given sufficient luminosity, the most promising SM Higgs discovery mechanism at the Tevatron for $m_{h_{\mathrm{SM}}} \lesssim 135 \mathrm{GeV}$ consists of $q \bar{q}$ annihilation into a virtual $V^{*}(V=W$ or $Z)$, where $V^{*} \rightarrow V h_{\mathrm{SM}}$ followed by a leptonic decay of the $V$ and $h_{\mathrm{SM}} \rightarrow b \bar{b}[61]$. The sum of the $W h_{\mathrm{SM}}$ and $Z h_{\mathrm{SM}}$ cross-sections is about $0.2-0.5 \mathrm{pb}$ in the mass region of interest $\left(100 \lesssim m_{h_{\mathrm{SM}}} \lesssim 135 \mathrm{GeV}\right)$, in which the dominant Higgs decay is $h_{\mathrm{SM}} \rightarrow b \bar{b}$. These processes lead to three main final states, $\ell \nu b \bar{b}, \nu \bar{\nu} b \bar{b}$ and $\ell^{+} \ell^{-} b \bar{b}$, that exhibit distinctive signatures on which the experiments can trigger (high $p_{T}$ leptons and/or missing $E_{T}$ ). The backgrounds are manageable and are typically dominated by vector-boson pair production, $t \bar{t}$ production and QCD dijet production. The signal efficiencies and backgrounds have all been estimated

\footnotetext{
${ }^{7} \mathrm{~A}$ critical comparison of varioms theoretical approaches to diffractive Higgs production can be found in rof. 558 .
} 
with both the CDF Run 1 detector simulation and with the simple SHW simulation [62]. Monte Carlo estimates have been used for the backgrounds everywhere except in the $\nu \bar{\nu} b \bar{b}$ channel, where there is a significant contribution from QCD $b \vec{b}$ dijet production. To be conservative: in ref. [62] the unknuwn QCD $b \bar{b}$ dijet background to the $\nu \bar{\nu} b \bar{b}$ channel has been taken to be equal in size to the sum of all other contributing background processes. In addition, the separation of signal from background was optimized using neural network techniques [63], resulting in a demonstrable gain in the significance of the Higgs signal for the $\ell \nu b \bar{b}$ and $\nu \bar{\nu} b \bar{b}$ channels [62]. The $b$-tagging efficiencies and the $b \bar{b}$ mass resolution play a key role in determining the ultimate efficiency and background rejection. Much work remains, using real data studies, to optimize the performance in both these areas.

For larger Higgs masses $\left(m_{h_{5 M}} \geq 135 \mathrm{GeV}\right)$ it is possible to exploit the distinct signatures presemt when the Higgs boson decay branching ratio to $W W^{(*)}$ becones appreciable. In this case, there are final states with $W W$ (from the gluon-fusion production of a single Higgs boson), and $W W W$ and $2 W W$ arising from associated vector boson- Higgs boson production. Three search channels were identified in ref. [62] as potentially sensitive at these high Higgs masses: like-sign dilepton plus jets ( $\ell^{ \pm} \rho^{ \pm} j$ ) events. high-p $p_{T}$ lepton pairs plus missing $E_{T}\left(\ell^{+} \ell^{-} \nu \bar{\nu}\right)$, and trilepton $\left(\ell^{ \pm} \ell^{\prime \prime} \ell^{\mp}\right)$ events. Of these the first (wo were found to be most sensitive. The strong angular correlations of the final state leptons resulting from $W W^{*}$ is one of the crucial ingredients for these discovery channels $[64.65,60$ ?

The inlegrated luminosity required per Tevatron experiment as a function of Higgs mass to wither exclude the SM Higgs boson at $95 \%$ CL or discover it at the $3 \sigma$ or $j \sigma$ level of significance for the SHW analyses with neural net selection (see ref. [62] for details), is shown in Fig. 7(a). These results are based on the combined statistical power of both the CDF and DQ experiments. The bands extend from the neural net result on the low side upward in required integrated luminosity by $30 \%$ to the high side, as an indication of the range of uncertainty in the $b$-tagging efficiency, $b \vec{b}$ mass resolution and background uncertainties. As the plots show, the required integrated luminosity increases rapidly with Higgs mass to $140 \mathrm{GeV}$, beyond which the high-mass channels play the dominant role. If $m_{h_{\mathrm{SM}}}=1.15 \mathrm{GeV}$, which lies just above the $95 \%$ CL exclusion limit achieved by LEP [11], then $j \mathrm{fb} \cdot{ }^{1}$ of integrated luminosity per experiment would provide sufficient data to see a $3 \sigma$ excess above background. With $15 \mathrm{fh})^{-1}$ of integrated luminosity per experiment, a $\bar{\sigma} \sigma$ discovery of the Higgs bowon would be possible.

The final result shows that for an integrated luminosity of $10 \mathrm{fb}^{-1}$. if the $5 \mathrm{M}$ Higgs boson mass lits: beyond the discovery reach of the Tevatron, then one can attain a $95 \%$ CL exclusion for masses up to about $180 \mathrm{GeV}$. Moreover, if the SM Higgs happens to be sufficiently light ( $\left.m h_{\mathrm{SM}} \lesssim 125 \mathrm{GeV}\right)$, then at tantalizing $3 \sigma$ effect will be visible with the same integrated luminositv. With about $25 \mathrm{fb}^{-1}$ of data. $3 \sigma$ evidence for the Higgs boson can be obtained for the entire Higgs mass range up to $180 \mathrm{GeV}$. However. the discovery reach is considerably more limited for a $5 \sigma$ Higgs boson signal. With $30 \mathrm{fb}^{-1}$ integrated luminosity delivered per detector, a $5 \sigma$ Higgs boson discovery may be possible for Higgs masses up to about $130 \mathrm{GeV}$, a significant extension of the LEP Standard Model Higgs search. The latter figure of merit is particularly significant, when applied to the search for the lightest. Higgs bosons of the MSSM We address this case in Section 3.5.3. 

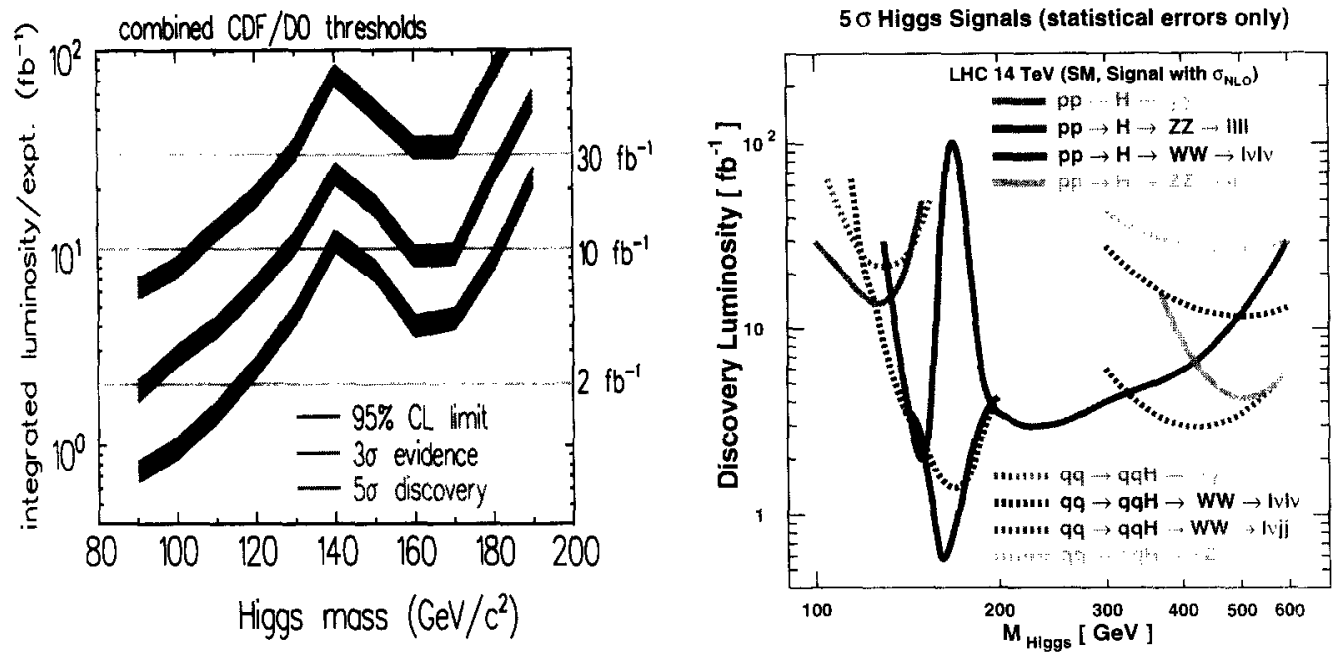

Figure 7: (a) The integrated luminosity required per Tevatron experiment, to either exclude a SM Higgs boson at $95 \%$ CL or observe it at the $3 \sigma$ or $5 \sigma$ level, as a function of the Higgs mass [62]. (b) Expected $5 \sigma$ discovery luminosity requirements for the SM Higgs boson at the LHC for one experiment, based on a study performed with CMS fast detector simulation, assuming statistical errors only [72]. The $g g$ and $W^{+} W^{-}$fusion processes are indicated respectively by the solid and dotted lines.

Other Iliggs signatures could help improve the sensitivity of the Higgs search at the Tevatron. In ref. [62], channels containing the $h_{\mathrm{SM}} \rightarrow \tau^{+} \tau^{-}$decay mode have not been studied, as the small branching ratio (less than $8 \%$ ) makes the corresponding signal rates small. Still, a significant improvement of $\tau$ lepton identification could lead to a viable Higgs signal in the Higgs mass region $120 \mathrm{GeV} \lesssim m_{h_{S M}} \lesssim$ $140 \mathrm{GeV}[66]$. Another possibility which has been explored is the detection of the Higgs boson via $t \bar{t} h_{\mathrm{SM}}$ production (the Higgs boson is radiated off the top-quark), followed by $h_{\mathrm{SM}} \rightarrow b \bar{b}$. Initial studies [67] suggested that this channel could be observable at the upgraded Tevatron for $m_{h_{\mathrm{SM}}} \lesssim 140 \mathrm{GeV}$, with a statistical significance comparable to the Higgs signals in the $W h_{\mathrm{SM}}$ and $Z h_{\mathrm{SM}}$ channels.

If a Higgs boson is discovered at the Tevatron, one can begin to measure some of its properties. The Higgs mass can be measured with an accuracy of about $2 \mathrm{GeV}$ [68]. However, the determination of the Higgs couplings to $W$ and $Z$ bosons and to $b \bar{b}$ will be model dependent and rather crude. To improve and expand the possible Higgs measurements and determine its phenomenological profile will require Higgs studies at the LHC. 


\subsubsection{Standard Model Higgs Boson Searches at the LHC}

Production rates for the Higgs boson in the Standard Model are significantly larger at the LHC 44,69 ). The dominant Higgs production process, gluon fusion, can be exploited in conjunction with a variety of other channels, e.g., WW/ZZ fusion of the Higgs boson and Higgs radiation off top quarks 70 i5]. Integrated luminosities between 30 and $100 \mathrm{fb}^{-1}$, achievable within the first few years of LHC operatiun. will be sufficient to cover the entire canonical Higgs mass range of the Standard Model up to valurs close to $1 \mathrm{TeV}$ with a significance greater than $5 \sigma$. The required LHC luminosities for a Higgs discovery in various channels are shown in fig. 7(b) [72]. Thus. there is no escape route for the SM Higgs bosm a.t the LHC.

The properties of the SM Higgs boson can be determined with some precision at the LHC: "The? $h_{\mathrm{SM}} \rightarrow Z Z^{(*)} \rightarrow \ell^{+} \ell^{-} \ell^{+} \ell^{-}$channel allows for an accurate Higgs mass determination of about 0 ). $1 \%$ (n) $120 \mathrm{GeV} \lesssim m_{h_{\mathrm{SM}}} \lesssim 400 \mathrm{GeV}$, assuming an integrated luminosity of $300 \mathrm{fb}^{-1}\lceil 76$. For larger Higgs masses, the precision in the Higgs mass measurement deteriorates clue to the effect of the inemasing Higgs width; nevertheless a $1 \%$ Higgs mass measurement is possible for $m_{h \mathrm{~s}} \simeq 700 \mathrm{GeV}$. The Higgs width can be extracted with a precision of 5 to $6 \%$ over the mass range 300 Tou GeV from the Brept-Wigny shape of the Higgs resonance [76]. Below $300 \mathrm{GeV}$, the instrumental resolution bromes larger than 1 hr. Higgs width, and the accuracy of the Higgs width measurement degrades. For example. the four-leptun

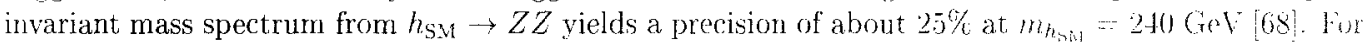
lower Higgs masses, indirect methods must be employed to measur ${ }^{2}$ the Higgs width

For Higgs masses below $200 \mathrm{GeV}$, a number of different Higgs decaly channels ran be studited at itu: LHC. The most relevant processes are

$$
\begin{aligned}
& g g \rightarrow h_{\mathrm{SM}} \rightarrow \gamma, \\
& g g \rightarrow h_{\mathrm{SM}} \rightarrow 1 \gamma^{(*)} \\
& q q \rightarrow q q V^{(*)} \gamma^{(*)} \rightarrow q q h_{\mathrm{SM}}, \quad h_{\mathrm{SM}} \rightarrow \gamma \gamma, \tau^{+} \tau^{\cdots} . l^{(*)} . \\
& g g, q \bar{q} \rightarrow t \bar{t} h_{\mathrm{SM}}, \quad h_{\mathrm{SM}} \rightarrow b \bar{b} . \gamma \gamma, W W^{(*)},
\end{aligned}
$$

where $V=W$ or $Z$. The gluon-gluon fusion mechanism is the dominant Higgs production mechanism at the LHC, yielding a total cross-section of about $30 \mathrm{pb}[15 \mathrm{pb}]$ for $m_{h_{\mathrm{SM}}}=120 \mathrm{GeV}\left[m_{h_{\mathrm{sM}}}=200 \mathrm{GeV}\right]$. One also has appreciable Higgs production via $V V$ electroweak gauge boson fusion, with a total crosssection of about $6 \mathrm{pb}[3 \mathrm{pb}]$ for the Higgs masses quoted above. The electroweak gauge boson fusion mechanism can be separated from the gluon fusion process by employing a forward jet tag and rentral jet vetoing techniques. Note that for $2 m_{W} \lesssim m_{h_{\mathrm{SM}}} \lesssim 2 m_{Z}$, the Higgs branching ratio to $Z Z^{*}$ is quite suppressed with respect to $W W$ (since one of the $Z$ bosons is off-shell). Hence, in this mass window. $h_{\mathrm{SM}} \rightarrow W^{+} W^{-} \rightarrow \ell^{+} \nu \ell^{-} \bar{\nu}$ is the main Higgs discovery channel [65], as exhibited in fig. ' $((\mathrm{b})$.

The cross-section for $t \bar{t} h_{\mathrm{SM}}$ production can be significant for Higgs masses in the intermediate mass range $[47], 0.8 \mathrm{pb}[0.2 \mathrm{pb}]$ at $m_{h_{\text {SM }}}=120 \mathrm{GeV}\left[m_{h_{S M}}=200\right.$ GeV $]$, although this coross-sertion falls faster 
with Higgs mass as compared to the gluon and gauge boson fusion mechanisms. Finally, we note that the preferred channel at the Tevatron, $q \bar{q} \rightarrow W h_{\mathrm{SM}} \rightarrow \ell \nu b \bar{b}$, is not a discovery mode at the LHC due in part to the larger background cross-sections at $\sqrt{s}=14 \mathrm{TeV}$. Nevertheless, with $300 \mathrm{fb}^{-1}$ of data, it may be possible to observe a SM Higgs signal (with $S / \sqrt{B} \geq 5$ ) if $m_{h_{\mathrm{SM}}} \lesssim 125 \mathrm{GeV}[77]$.

The measurements of Higgs decay branching ratios can be used to infer the ratios of the Higgs couplings, and provide an important first step in clarifying the nature of the Higgs boson [78-80]. These can be extracted from a variety of Higgs signals that are observable over a limited range of Higgs masses. In the mass range $110 \mathrm{GeV} \lesssim m_{h_{S M}} \lesssim 150 \mathrm{GeV}$, the Higgs boson can be detected [with $100 \mathrm{fb}^{-1}$ of data] in the $\gamma \gamma$ and the $\tau^{+} \tau^{-}$channels indicated above. (The $\mu^{+} \mu^{-}$channel was considered in ref. [80]. With $300 \mathrm{fb}^{-1}$ of data, a $3 \sigma$ excess above background may be possible for $110 \mathrm{GeV} \lesssim m_{\iota_{\mathrm{SM}}} \lesssim 140 \mathrm{GeV}$.) For $n_{h_{\mathrm{SM}}} \gtrsim 130 \mathrm{GeV}$, the Higgs boson can also be detected in gluon-gluon fusion through its decay to $1{ }^{\prime \prime} W^{(*)}$, with both final gauge bosons decaying leptonically [81], and to $Z Z^{(*)}$ in the four-lepton decay mode $[70,74]$. There is additional sensitivity to Higgs production via. $V V$ fusion followed by its decay to IV $W^{(*)}$ for $m_{h_{\mathrm{SM}}} \gtrsim 120 \mathrm{GeV}$. These data can be used to extract the ratios of the Higgs partial widths to gluon pairs, photon pairs, $\tau^{+} \tau^{-}$, and $W^{+} W^{-}[78,79]$. The expected accuracies in Higgs width ratios, partial widths, and the total Higgs width are exhibited in fig. 8 . These results are obtained under the assumption that the partial Higgs widths to $W^{+} W^{-}$and $Z Z$ are fixed by electroweak gauge invariance, and the ratio of the partial Higgs widths to $b \vec{b}$ and $\tau^{1} \tau$ are fixed by the universality of Higgs couplings 1. down-type fermions. One can then extract the total Higgs width under the assumption that all other unobserved modes, in the Standard Model and beyond, possess small branching ratios of order $1 \%$. Finally, we note that the specific Lorentz structure predicted for the $h_{\mathrm{SM}} W^{+} W^{-}$coupling by the Higgs mechanism can be tested in angular correlations between the spectator jets in $W W^{\circ}$ fusion of the Higgs boson at the LIIC [79].

With an integrated luminosity of $100 \mathrm{fb}^{-1}$ per experiment, the relative accuracy expected at the LHC for various ratios of Higgs partial widths $\Gamma_{2}$ (or equivalently, ratios of Higgs squared-couplings) range from $10 \%$ to $30 \%$, as shown in fig. 8 . The ratio $\Gamma_{T} / \Gamma_{W}$ measures the coupling of down-type fermions relative to the Higgs couplings to gauge bosons. To the extent that the one-loop $h_{\mathrm{SM}} \gamma \gamma$ amplitude is dominated by the $W$-loop, the partial width ratio $\Gamma_{\gamma} / \Gamma_{\gamma}$ probes the same relationship. In contrast, under the usual assumption that the one-loop $h_{\mathrm{SM}} g g$ amplitude is dominated by the top-quark loop, the ratio $\Gamma_{g} / \Gamma_{W}$ probes the coupling of up-type fermions relative to the $h_{\mathrm{SM}} W W$ coupling. In addition, one can measure the $h_{\mathrm{SM}} t \bar{t}$ coupling directly by making use of the $t \bar{t} h_{\mathrm{SM}}$ production mode at the LHC. Recent studies suggest that for an integrated luminosity of $100 \mathrm{fb}^{-1}$, this signal is viable for the $h_{\mathrm{SM}} \rightarrow b \bar{b}[82,83]$ and $h_{\mathrm{SM}} \rightarrow \tau^{+} \tau^{-}[84]$ decay modes if $m_{h_{\mathrm{SM}}} \lesssim 130-140 \mathrm{GeV}$. and for the $h_{\mathrm{SM}} \rightarrow W W^{(*)}$ decay mode for $130 \lesssim m_{h_{\mathrm{SM}}} \lesssim 200 \mathrm{GeV}[85]$. In this way, one expects to be able to measure the Higgs-top quark Yukawa coupling with a relative accuracy in the range of 10-20\%.

Finally, the measurement of the triple Higgs self-coupling with an accuracy of order $25 \%$ can be obtained, in a very limited Higgs mass window, with $3000 \mathrm{fb}^{-1}$ of data $[86]$ (which requires at least a fartor-of-ten luminosity upgrade of the LHC [87]). 
width ratios

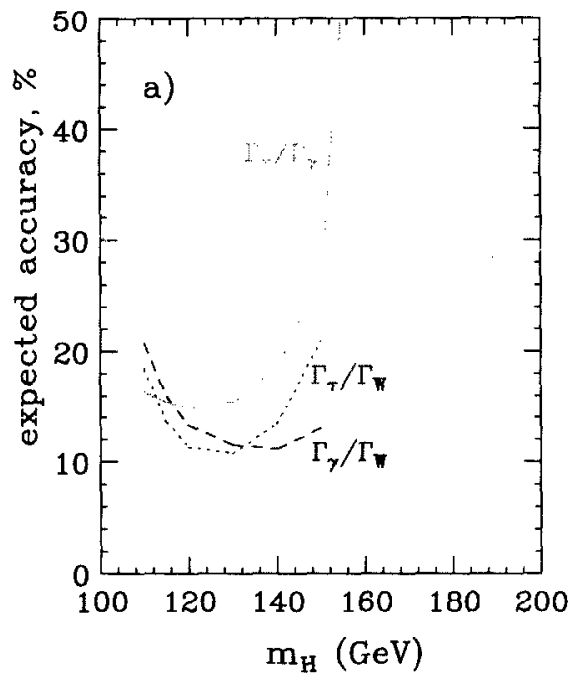

(partial) widths

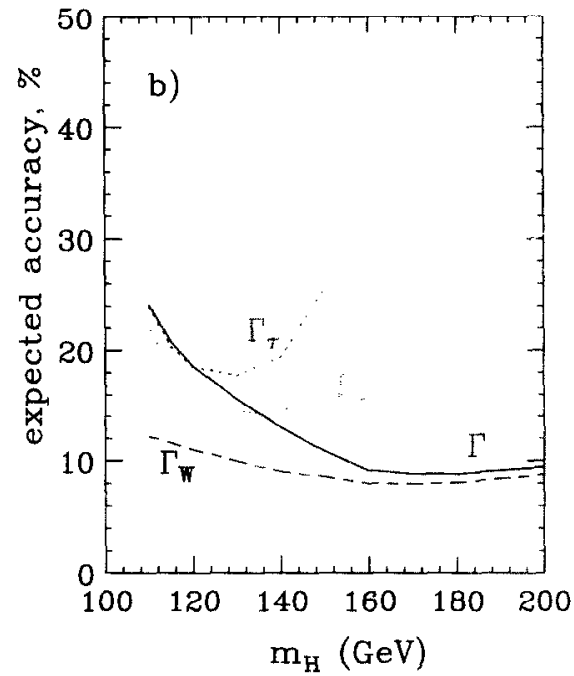

Figure 8: Relative accuracy expected at the LHC with $200 \mathrm{fb}^{-1}$ of data for (a) various ratios of Higgs boson partial widt hs and (b) the indirect determination of partial and total widths. Expectations for width ratios assume $W$. $Z$ universality: indirect width measurements also assume $b, \tau$ universality and a small branching ratio for unobserved modes. Taken from the parton-level analysis of ref. [78].

\subsection{Standard Model Higgs Boson Searches at the LC}

The next generation of high energy $e^{+} e^{-}$linear colliders is expected to operate at energies from $300 \mathrm{GeV}$ up to about $1 \mathrm{TeV}$ (JLC, NLC, TESLA), henceforth referred to as the LC [88 90]. The possibility of a multi-TeV linear collider operating in an energy range of $3-5 \mathrm{TeV}$ (CLIC) is also under study 91 ?. With the expected high luminosities up to $1 \mathrm{ab}^{-1}$, accumulated within a few years in a clean experimental environment, these colliders are ideal instruments for reconstructing the mechanism of elcctroweak symmetry breaking in a comprehensive and conclusive form.

Weakly-coupled electroweak symmetry breaking dynamics involving an elementary scalar Higgs tield (an be established experimentally in three steps. First, the Higgs boson must be observed clearly and unambiguously, and its basic properties--mass. width, spin and $\mathrm{C}$ and $\mathrm{P}$ quantum numbers- must be determined. Second, the couplings of the Higgs boson to the $W^{ \pm}$and $Z$ bosons and to leptons and 

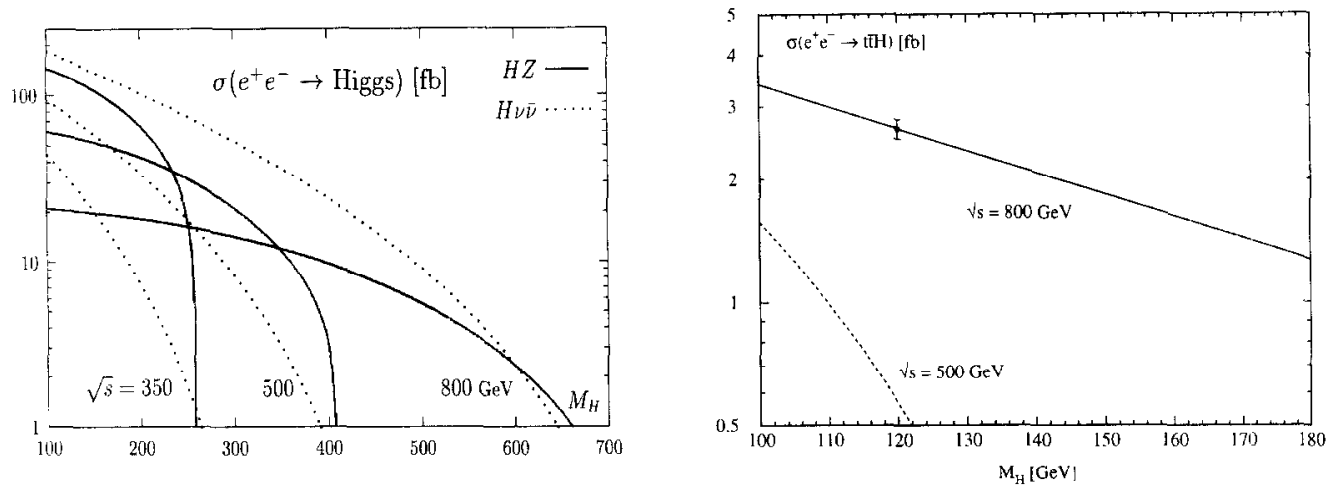

Figure 9: (a) The Higgs-strahlung and WW fusion production cross-sections as functions of $m_{h_{\mathrm{SM}}}$ for $\sqrt{\mathrm{s}}=350 \mathrm{GeV}$, $500 \mathrm{GeV}$ and $800 \mathrm{GeV}$. (b) The cross-section for $e^{+} e^{-} \rightarrow t \bar{t} h_{\mathrm{SM}}$, including NLO QCD corrections [102], as a function of $m_{h_{\mathrm{SM}}}$ for $\sqrt{s}=500 \mathrm{GeV}$ and $800 \mathrm{GeV}$ with the expected experimental accuracy for $m_{h_{\mathrm{SM}}}=120 \mathrm{GeV}$ shown by the dot with error bar for an integrated luminosity of $1000 \mathrm{fb}^{-1}$. Taken from ref. [92].

quarks must be measured. Demonstrating that these couplings scale with the mass of the corresponding particle would provide critical support for the Higgs mechanism based on scalar dynamics as the agent responsible for generating the masses of the fundamental particles. Finally, the Higgs potential must be reconstructed by measuring the self-coupling of the Higgs field. The specific form of the potential shifts the ground state to a non-zero value, thereby providing the mechanism for electroweak symmetry breaking based on the self-interactions of scalar fields. Essential elements of this program can be realized at a high-luminosity $e^{+} e^{-}$linear collider, and high-precision analyses of the Higgs boson are possible in these machines [92-94].

The main production mechanisms of the SM Higgs boson at the LC are the Higgs-strahlung process $[95,25], e^{+} e^{--} \rightarrow Z h_{\mathrm{SM}}$, and the $W W$ fusion process [96] $e^{+} e^{-} \rightarrow \bar{\nu}_{e} \nu_{e} W^{*} W^{*} \rightarrow \bar{\nu}_{e} \nu_{e} h_{\mathrm{SM}}$. With an accumulated luminosity of $500 \mathrm{fb}^{-1}$, about $10^{5}$ Higgs bosons can be produced by Higgs-strahlung in the theoretically preferred intermediate mass range below $200 \mathrm{GeV}$. As $\sqrt{s}$ is increased, the cross-section for the Higgs-strahlung process decreases as $s^{-1}$ and is dominant at low energies, while the cross-section for the $W W$ fusion process grows as $\ln \left(s / m_{h_{S M}}^{2}\right)$ and dominates at high energies (see ref. [97] for a convenient form for the corresponding cross-sections), as shown in fig. $9(\mathrm{a}) .{ }^{8}$ The $Z Z$ fusion mechanism,

\footnotetext{
${ }^{8}$ The cross-sections shown in fig. $9(\mathrm{a})$ are based on a tree-level computation. The complete one-loop electroweak corrections for $e^{+} e^{-} \rightarrow Z h_{\mathrm{SM}}$ have been computed [98,97], whereas only the leading one-loop corrections to the vector boson fusion process are known [99].
} 
$\epsilon^{r} e^{-} \rightarrow e^{+} e^{-} Z^{*} Z^{*} \rightarrow \epsilon^{+} \epsilon^{-} h_{\mathrm{SM}}$, also contributes to Higgs production, with a cross-section suppressert with respect to that of $W W$ fusion by a factor of $16 \cos ^{4} \theta_{W^{\prime}} /\left[1+\left(1-4 \sin ^{2} \theta_{W^{\prime}}\right)^{2}\right] \simeq 9.4$. The (rosissections for the Higgs-strahlung and the $W W$ fusion processes are shown in fig. 9(a) for three values ol $\sqrt{s}$. The Higgs-strahlung process, $e^{+} e^{-} \rightarrow Z h_{S M}$, with $Z \rightarrow \ell^{+} t^{-}$. offer's a very distinctive signature. For $\sqrt{s}=350$ and $500 \mathrm{GeV}$ and an integrated luminosity of $500 \mathrm{fh}^{-1}$. this mensures the observation of $\mathrm{th}$. SM Higgs boson up to the production kinematical limit independently of its decay [92]. At $\sqrt{s}=500$ GeV, the Higgs-strahlung and the WW fusion processes have approximately the same cruss-sections. $\mathcal{O}(50 \mathrm{fb})$, for $100 \mathrm{GeV} \lesssim m_{h_{5 M}} \lesssim 200 \mathrm{GrV}$. At $\sqrt{s}=800 \mathrm{Gel}$ with $500 \mathrm{fh}^{-1}$ of dat.a, the analysis if

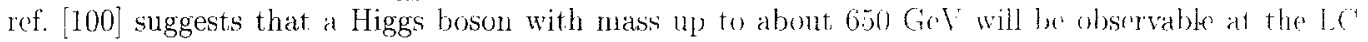
Finally, the process $f^{+} f \rightarrow t h_{S M}$ [101] yields a distinctive signature consisting of rwo If bessons and four $b$-quark jets, and can be observed at the LC given sufficient atergy and luminosity if the Higes mass is not too large. The QCD-corrected cross-sections for this process $[102]$ for $\sqrt{s}=500 \mathrm{G}+1 \cdot$ atud $800 \mathrm{GeV}$ are shown in fig. $9(\mathrm{~b})$.

The phenomenological profile of the Higgs boson can be deternined by precision measurements. For example, consider the case of $m_{h_{5,1}}=120 \mathrm{GeV}$ at the $\mathrm{IC}$ with $\sqrt{s}=350 \mathrm{GeV}$ and $500 \mathrm{fth}^{-1}$, if data [92]. The spin and parity of the Higgs boson can be determined unambiguously from the ste't] onset of the excitation curve in Higgs-strahlung near the threshold (see fig. 10(a) [103]) and the angular correlations in this process [104]. By measuring final state angular distributions and various angular and polarization asymmetries. one ran check whether the Higgs boson is a state of definite CP. or whether it rxhibits CP-violating behavior in its production and/or decays [105]. The Higgs mass can be measured to an accuracy of $40 \mathrm{MeV}$ by reconstructing the Higgs boson in $Z h_{\text {sM }}$ production and combining the results from the various final state channels. The Higgs width can be inferred in a model-independern way. with an accuracy of about $6 \%$, by combining the partial width $6 \mathrm{~W}^{-} \mathrm{W}^{-}$, accessible in the vector boson fusion process, with the $W^{*}$ decay branching ratio. Similar results (with precisions within a factor of two of those quoted above) are obtained for larger Higgs masses in the intermediate mans regime. The $h_{\mathrm{SM}} Z Z, h_{\mathrm{SM}} Z_{\gamma}$ and $h_{\mathrm{SM}} t \bar{t}$ couplings can be determined (with some sensitivity to pussible anomalous couplings, if present) by the optimal-observable method [106], which makes optimal use of the polarizcd angular distributions and asymmetries for Higgs-strahlung and $h_{\text {sm }}+\bar{t}$ produrtion.

Higgs decay branching ratios can be measured very precisely for $m_{h s} \leqslant 1,50$ GoV 107 110. When such measurements are combined with measurements of Higgs production cross-sections, the absolut: values of the Higgs couplings to the $W^{ \pm}$and $Z$ gauge bosons and the Yukawa couplings to leptons and quarks can be determined to a few percent in a model-independent way. In addition, the Higgs-tup, quark Yukawa coupling can be inferred from the cross-section for Higgs emission off $t \vec{t}$ pairs $[102.11 .1\}$. As an example, Table 1 exhibits the anticipated fractional uncertainties in the measurements of Higgs branching ratios for $m_{h_{\mathrm{SM}}}=120 \mathrm{GeV}$ at the $\mathrm{LC}{ }^{9}$ Lsing this data. a program HFITTER was developed in

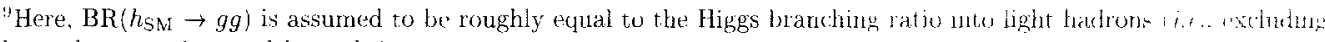
hadrons that contain $c$ and $b$ (quarks).
} 

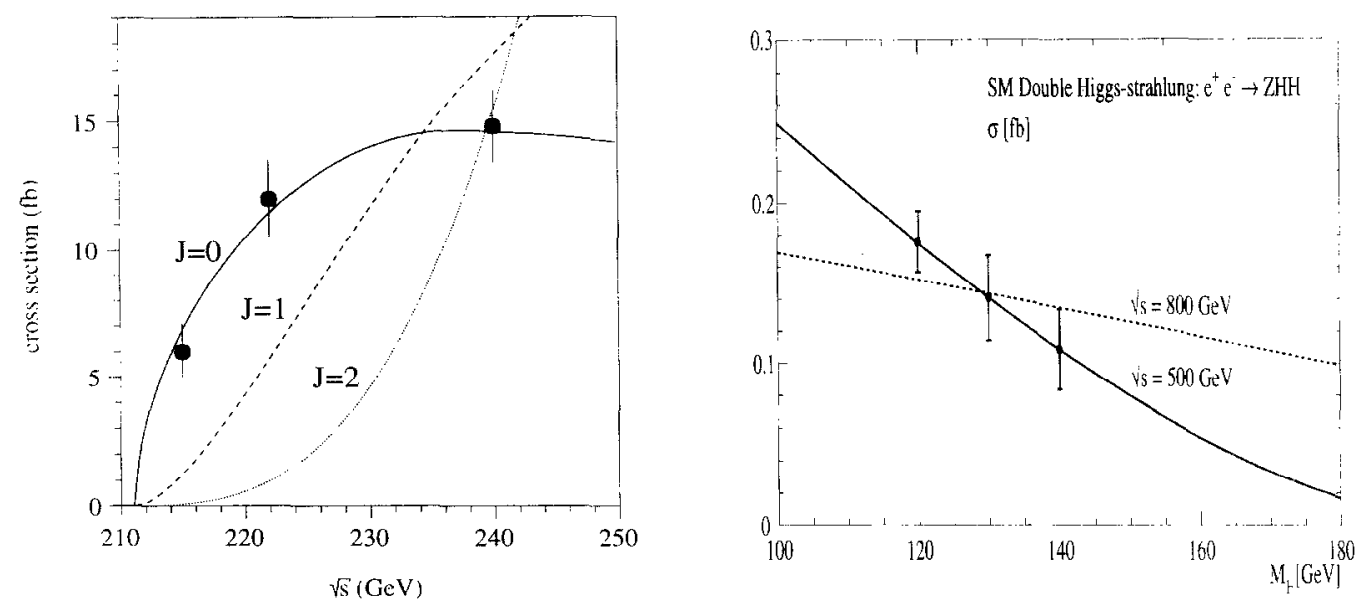

Figure 10: (a) Simulated measurement of the $e^{+} e^{-} \rightarrow Z h_{\mathrm{SM}}$ cross-section for $m_{h_{\mathrm{SM}}}=120 \mathrm{GeV}$ with $20 \mathrm{fb}^{-1}$ per point at three center of mass energies compared to the predictions for spin-0 (solid line) and typical examples of spin-1 (dashed line) and spin-2 (dotted line) [103]. (b) Cross-section for the double Higgs-strahlung process $e^{+} e^{-} \rightarrow Z h_{\mathrm{SM}} h_{\mathrm{SM}}$ at $\sqrt{s}=500 \mathrm{GeV}$ (solid line) and $800 \mathrm{GeV}$ (dashed line) [114]. The data points show the accuracy for 1 ab"-1.

\begin{tabular}{|c||c|c|}
\hline Higgs coupling & $\delta \mathrm{BR} / \mathrm{BR}$ & $\delta g / g$ \\
\hline \hline$h W W$ & $5.1 \%$ & $1.2 \%$ \\
$h Z Z$ & - & $1.2 \%$ \\
\hline$h t t$ & - & $2.2 \%$ \\
$h b b$ & $2.4 \%$ & $2.1 \%$ \\
$h c c$ & $8.3 \%$ & $3.1 \%$ \\
$h \tau \tau$ & $5.0 \%$ & $3.2 \%$ \\
$h \mu \mu$ & $\sim 30 \%$ & $\sim 15 \%$ \\
\hline$h g g$ & $5.5 \%$ & \\
$h \gamma \gamma$ & $16 \%$ & \\
$h h h$ & & $\sim 20 \%$ \\
\hline
\end{tabular}

Table 1: Expected fractional uncertainties for measurements of Higgs branching ratios $[\mathrm{BR}(h \rightarrow X \bar{X})]$ and couplings $\left\lfloor g_{h \times X}\right]$, for various choices of final state $X \bar{X}$, assuming $m_{h}=120 \mathrm{GeV}$ at the LC. In all but four cases, the resultis shown are based on $500 \mathrm{fb}^{-1}$ of data at $\sqrt{s}=500 \mathrm{GeV}$ [107]. Results for $h \gamma \gamma[108], h t \bar{t}[107], h \mu \mu[109]$ and $h h h[114,115]$ are based on $1 \mathrm{ab}^{-1}$ of data at $\sqrt{s}=500 \mathrm{GeV}$ (for $\gamma \gamma$ and $h h$ ) and $\sqrt{s}=800 \mathrm{GeV}$ (for $t t$ and $\mu \mu$ ), respectively. 
ref. [107] to perform a Standard Model global fit based on the measurements of the $Z h_{\mathrm{SW}}$, wi $h_{\mathrm{SN}}$ and $t \bar{t} h_{S \mathrm{M}}$ cross-sections and the Higgs branching ratios listed in Table 1 . The output of the program is a set of Higgs couplings along with their fractional uncertainties (which are also exhibited in Table 1). These results should be considered representative of what can eventually be achieved at the LC. For example, a comprehensive analysis of LC Higgs data will have to take into account the relevant electroweak loop corrections (which are not presently included in HFITTER). One should also note that theoretical uncertainties for the predicted Higgs couplings have not been taken into account in this analysis. The theoretical uncertainty in $g_{h_{S M} c \bar{c}}$ is the most significant among the channels listed in 'lable 1. due to the uncertainties in $c$ quark-mass and in $\alpha_{s}$ (which governs the running of the quark masses from the quark mass to the Higgs mass). Ref. [112] estimates a theoretical fractional uncertainty in $g_{h i c}$ of about $12 \%$. significantly greater than the experimental uncertainty listed in Table 1 . In contrast. the theoretical fractional uncertainty in $g_{h b \bar{b}}$ is about $1.8 \%$ due to the uncertainty of the b-quark mass. Although this is less than the anticipated experimental uncertainty, it should not be neglected in the determination of the overall $g_{h_{\mathrm{SM}} b \bar{b}}$ uncertainty. The theoretical uncertainties in the other channels listed above are nut: significant compared to the quoted experimental uncertainties.

The measurement of the Higgs self-couplings is a very ambitious task that requires the hightest luminosities possible at the $\mathrm{LC}$, which possess unique capabilities for addressing this question. The trilinear Higgs self-coupling can be measured in double Higgs-strahlung, in which a virtual Higgs buson splits into two real Higgs particles in the final state [113]. A simulation based on $1 \mathrm{ab}^{-1}$ of data is exhibited in fig. 10(b) [114]. In this way, for $m_{h_{\mathrm{SM}}}=120 \mathrm{GeV}$, the cubic term of the scalar potential can be established at the LC with a precision of about $20 \%[114,115]$. Such a measurement is a prerequisite for determining the form of the Higgs potential that is responsible for spontaneous electroweak symmetry breaking generated by scalar sector dynamics. Finally, the total SM Higgs width can be obtained indirectly by using $\Gamma_{\text {tot }}=\Gamma_{h_{\mathrm{SM}} W W} / \mathrm{BR}\left(h_{\mathrm{SM}} \rightarrow W^{*} W^{*}\right)$. The partial width is proportional to $g_{h_{S M}}^{\prime} i \mathrm{H}$. so the fractional uncertainty in $\Gamma_{\text {tot }}$ can be obtained from the results of Table 1 . For $m_{h_{s, 3}} \leqslant 150 \mathrm{Ge}$ : an accuracy in the range of $5-10 \%$ can be achieved for the total Higgs width [116]

If the SM Higgs mass is above $150 \mathrm{GeV}$, then the precision determination of Higgs couplings will have to be reconsidered. The Higgs branching ratios into $c \bar{c}$, $g g$ and $\tau^{+} \tau^{-}$are now too small to he accurately measured. Due to the growing importance of the $W W$ and $Z Z$ morles. one can perform a precision measurement of the Higgs branching ratios to $W W$ and $Z Z$, while the precision of the $b \bar{b}$ branching ratio is significantly reduced as $m_{h_{5 M}}$ increases to $200 \mathrm{GeV}$ and beyond [117]. Moreoves. dut to the rapid decline of $t \bar{t} h_{\mathrm{SM}}$ production cross-section with increasing $m_{t_{s: \mathrm{M}}}$, the Higgs-top quark Yukawa coupling cannot be extracted until $m_{h_{\mathrm{SM}}} \geq 2 m_{t}$, at which point the tith $h_{\mathrm{SM}}$ coupling can be obtained by observing Higgs bosons produced by vector boson fusion which subsequently decay to tt. The analysis of Ref. [118] finds that at the LC with $\sqrt{s}=800 \mathrm{GeV}$ and $1 \mathrm{ab}^{-1}$ of data, the $t \bar{t} h_{\mathrm{s} \text { M }}$ Yukawa coupling call be determined with an accuracy of about $10 \%$ for a Higgs mass in the range $350-500 \mathrm{GeV}$. The total Higgs width can be obtained directly from measuring the Higgs boson line-shape if $m_{h_{S M}} \gtrsim 200 \mathrm{GN}$.

The $e^{+} e^{-}$linear collider with center-of-mass energy $\sqrt{s}$ can also be designed to operate in it $\gamma$ 
collision mode. This is achieved by using Compton backscattered photons in the scattering of intense laser photons on the initial polarized $e^{ \pm}$beams $[119,120]$. The resulting $\gamma \gamma$ center of mass energy is peaked for proper choices of machine parameters at about $0.8 \sqrt{s}$. The luminosity achievable as a function of the photon beam energy depends strongly on the machine parameters (in particular, the (hoice of laser polarizations). The $\gamma \gamma$ collider provides additional opportunities for Higgs physics [120 124]. The Higgs boson can be produced as an $s$-channel resonance in $\gamma \gamma$ collisions, and one can perform independent measurements of various Higgs couplings. For example, the product $\Gamma\left(h_{\mathrm{SM}} \rightarrow\right.$ $\gamma \gamma) \operatorname{BR}\left(h_{\mathrm{SM}} \rightarrow b \bar{b}\right)$ can be measured with a statistical accuracy of about $2-10 \%$ for $120 \mathrm{GeV} \lesssim m_{h_{\mathrm{SM}}} \lesssim$ $160 \mathrm{GeV}$ with about $50 \mathrm{fb}^{-1}$ of data [122-124]. In order to reach such a precision, it is critical to control the ovcrwhelming two-jct background (with efficient $b$-tagging) and overcome the irreducible $\gamma \gamma \rightarrow b \bar{b}$ background by optimal use of the polarization of the photon beams and by judicious kinematic cuts. Knowledge of the QCD corrections to signal and background processes is essential for this task [122,125].

lising values for $\mathrm{BR}\left(h_{\mathrm{SM}} \rightarrow b \bar{b}\right)$ and $\mathrm{BR}\left(h_{\mathrm{SM}} \rightarrow \gamma \gamma\right)$ measured at the $e^{+} e^{--}$linear collider. one can obtain a value for the total Higgs width with an error dominated by the expected error in BR $\left(h_{S M} \rightarrow \gamma \gamma\right)$. For heavier Higgs bosons, $m_{h_{\mathrm{SM}}} \gtrsim 200 \mathrm{GeV}$, the total Higgs width can in principle be measured directly by tuning the collider to scan across the Higgs resonance. One can also use the polarization of the photon beams to measure various asymmetries in Higgs production and decay, which are sensitive to the CP quantum numbers of the Higgs boson [123].

\section{The Higgs Bosons of Low-Energy Supersymmetry}

Electroweak symmetry breaking dynamics driven by a weakly-coupled elementary scalar sector requires a mechanism for the stability of the electroweak symmetry breaking scale with respect to the Planck scale [5]. Supersymmetry-breaking effects, whose origins may lie at energy scales much larger than $1 \mathrm{TeV}$, can induce a radiative breaking of the electroweak symmetry due to the effects of the large Higgs-top quark Yukawa coupling [126]. In this way, the origin of the electroweak symmetry breaking scale is intimately ticd to the mechanism of supcrsymmetry brcaking. Thus, supcrsymmetry provides an explanation for the stability of the hierarchy of scales, provided that supersymmetry-breaking masses in the low-energy effective electroweak theory are of $\mathcal{O}(1 \mathrm{TeV})$ or less [5].

A fundamental theory of supersymmetry-breaking is presently unknown. Nevertheless, one can parameterize the low-energy theory in terms of the most general set of soft-supersymmetry-breaking terms [127]. The simplest realistic model of low-energy supersymmetry is a minimal supersymmetric extension of the Standard Model (MSSM), which employs the minimal supersymmetric particle spectrum. However, even in this minimal model with the most general set of soft-supersymmetry-breaking terms, more than 100 new supersymmetric parameters are introduced [128]. Fortunately, most of these parameters have no impact on Higgs phenomenology. Thus, we will focus primarily on the Higgs sector of the MSSM and identify the parameters that govern the main properties of the Higgs bosons. 


\subsection{The Tree-Level Higgs Sector of the MSSM}

Both hypercharge $Y=-1$ and $Y=+1$ complex Higgs doublets are required in any Higgs sectol of an anomaly-free supersymmetric extension of the Standard Model. The supersymmetric structure of the theory also requires (at least) two Higgs doublets to gencrate mass for both "up"-type and "down"-type quarks (and charged leptons) [6]. Thus, the MSSM contains the particle spectrum of a two-Higgs-doublet extension of the Standard Model and the corresponding supersymmetric partners.

The two-doublet Higgs sector [129] contains eight scalar degrees of freedom: one complex $r:=\ldots 1$ doublet, $\Phi_{\boldsymbol{d}}=\left(\Phi_{d}^{0}, \Phi_{d}^{-}\right)$and one complex $\mathrm{V}=+1$ doublet, $\boldsymbol{\Phi}_{\boldsymbol{u}}=\left(\Phi_{u}^{+} . \Phi_{u}^{0}\right)$. The notation reflects the form of the MSSM Higgs sector coupling to fermions: $\Phi_{d}^{0}\left[\Phi_{u}^{0}\right]$ romples exclusively to down-type [uptype fermion pairs. When the Higgs potential is minimized the noutral components of the Higgs fields acquire vacuum expectation values: ${ }^{10}$

$$
\left\langle\boldsymbol{\Phi}_{\mathrm{d}}\right\rangle=\frac{1}{\sqrt{2}}\left(\begin{array}{c}
v_{\mathrm{d}} \\
0
\end{array}\right), \quad\left\langle\boldsymbol{\Phi}_{\mathrm{u}}\right\rangle=\frac{1}{\sqrt{2}}\left(\begin{array}{c}
0 \\
\iota_{n}
\end{array}\right) .
$$

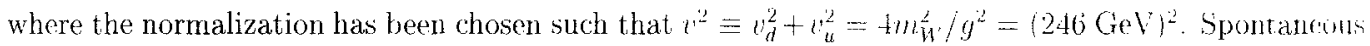
electroweak symmetry breaking results in three Goldstone bosuns, which are absorbed and become: th: longitudinal components of the $\mathfrak{H}^{+}$and $Z$. The remaining five physical Higgs particles runsist if a rlarged Higgs pair

$$
H^{ \pm}=\Phi_{d}^{ \pm} \sin \theta+\Phi_{u}^{+} \cos \gamma
$$

one CP-odd scalar

and two CP-even scalars:

$$
4=\sqrt{2}\left(\operatorname{Im} \Phi_{d}^{0} \sin \beta+\operatorname{Im} \Phi_{u}^{u} \cos \beta\right)
$$

$$
\begin{aligned}
& h=-\left(\sqrt{2} \operatorname{Re} \Phi_{d}^{0}-v_{d}\right) \sin a+\left(\sqrt{2} \operatorname{Re} \Phi_{u}^{0}-r_{n}\right) \cos \alpha . \\
& H=\left(\sqrt{2} \operatorname{Re} \Phi_{d}^{0}-v_{d}\right) \cos \alpha+\left(\sqrt{2} \operatorname{Re} \Phi_{u}^{0}-\iota_{n}\right) \sin \alpha .
\end{aligned}
$$

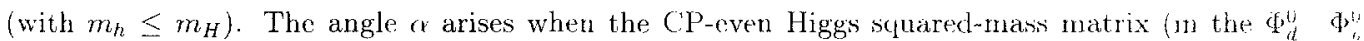
basis) is diagonalized to obtain the physical CP-even Higgs states (explicit formulae will be given below)

The supersymmetric structure of the theory imposes constraints on the Higgs sector of the morld For example, the Higgs self-interactions are not independent parameters: they can be expressed in texmi of the electroweak gauge coupling constants. As a result, all Higgs sector parameters at tree-level arr determined by two free parameters: the ratio of the two neutral Higgs field vacuum expectation valurs.

$$
\tan \beta \equiv \frac{u_{t t}}{u_{t},}
$$

\footnotetext{
${ }^{10}$ The phases of the Higgs fields can be chosen such that the vacuum expectation values are real and pusitive. Thint. is, the tree-level MSSM Higgs sector conserves CP, which implies that the neutral Higgs nass eigenstates possess ditinite CP quantum numbers.
} 
and one Higgs mass, conveniently chosen to be $m_{A}$. In particular,

$$
m_{I^{ \pm}}^{2}=m_{A}^{2}+m_{W}^{2}
$$

and the CP-even Higgs bosons $h$ and $H$ are eigenstates of the following squared-mass matrix

$$
\mathcal{M}_{0}^{2}=\left(\begin{array}{cc}
m_{A}^{2} \sin ^{2} \beta+m_{Z}^{2} \cos ^{2} \beta & -\left(m_{A}^{2}+m_{Z}^{2}\right) \sin \beta \cos \beta \\
-\left(m_{A}^{2}+m_{Z}^{2}\right) \sin \beta \cos \beta & m_{A}^{2} \cos ^{2} \beta+m_{Z}^{2} \sin ^{2} \beta
\end{array}\right) .
$$

The eigenvalues of $\mathcal{M}_{0}^{2}$ are the squared-masses of the two CP-even Higgs scalars

$$
m_{H, h}^{2}=\frac{1}{2}\left(m_{A}^{2}+m_{Z}^{2} \pm \sqrt{\left(m_{A}^{2}+m_{Z}^{2}\right)^{2}-4 m_{Z}^{2} m_{A}^{2} \cos ^{2} 2 \beta}\right),
$$

and $\alpha$ is the angle that diagonalizes the CP-even Higgs squared-mass matrix. From the above results, one obtains:

$$
\cos ^{2}(\beta-\alpha)=\frac{m_{h}^{2}\left(m_{Z}^{2}-m_{h}^{2}\right)}{m_{A}^{2}\left(m_{H}^{2}-m_{h}^{2}\right)} .
$$

In the convention where $\tan \beta$ is positive (i.e., $0 \leq \beta \leq \pi / 2$ ), the angle $\alpha$ lies in the range $-\pi / 2<\alpha \leq 0$.

An important consequence of eq. (12) is that there is an upper bound to the mass of the light CP-even Higgs boson, $h$. One finds that:

$$
m_{h} \leq m_{z}|\cos 2 \beta| \leq m_{z}
$$

This is in marked contrast to the Standard Model, in which the theory does not constrain the value of $m_{h_{\mathrm{SM}}}$ at tree-level. The origin of this difference is easy to ascertain. In the Standard Model, $m_{h_{\mathrm{SM}}}^{2}==\frac{1}{2} \lambda v^{2}$ is proportional to the Higgs self-coupling $\lambda$, which is a free parameter. On the other hand. all Higgs self-coupling parameters of the MSSM are related to the squares of the electroweak gauge couplings.

Note that the Higgs mass inequality [eq. (14)] is saturated in the limit of large $m_{A}$. In the limit of $m_{A} \gg m_{Z}$, the expressions for the Higgs masses and mixing angle simplify and one finds

$$
\begin{aligned}
m_{h}^{2} & \simeq m_{Z}^{2} \cos ^{2} 2 \beta, \\
m_{H}^{2} & \simeq m_{A}^{2}+m_{Z}^{2} \sin ^{2} 2 \beta, \\
m_{H^{ \pm}}^{2} & =m_{A}^{2}+m_{W}^{2}, \\
\cos ^{2}(\beta-\alpha) & \simeq \frac{m_{Z}^{4} \sin ^{2} 4 \beta}{4 m_{A}^{4}} .
\end{aligned}
$$

Two consequences are immediately apparent. First, $m_{A} \simeq m_{H} \simeq m_{H^{ \pm}}$, up to corrections of $\mathcal{O}\left(m_{Z}^{2} / m_{A}\right)$. Second, $\cos (\beta-\alpha)=0$ up to corrections of $\mathcal{O}\left(m_{Z}^{2} / m_{A}^{2}\right)$. This limit is known as the deconpling limit [130] 
because when $m_{A}$ is large, there exists an effective low-energy theory below the scale of $m_{A}$ in which the effective Higgs sector consists only of one CP-even Higgs boson. h. As we shall demonstrate below. the

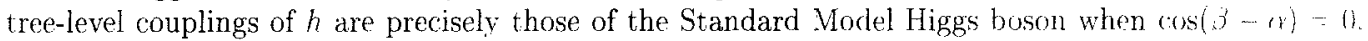
From eq. (18), one can also derive:

$$
\cot \alpha=-\tan \beta-\frac{2 m_{Z}^{2}}{m_{A}^{2}} \tan \beta \cos 2 \beta+o\left(\frac{m_{Z}^{t}}{m_{t}^{4}}\right)
$$

This result will prove useful in evaluating the CP-even Higgs boson couplings to frrmion pairs in the decoupling limit.

The phenomenology of the Higgs sector depends in detail wn the various couplings of the Higgs bosons to gauge bosons, Higgs bosons and fermions. The couplings of the two CP-even Higgs bosons to $W$ and $Z$ pairs are given in terms of the angles $\alpha$ and $\beta$ by

$$
g_{h Y v}=g_{V} m_{V} \sin (\beta-\alpha) . \quad g_{H V}=g_{V} m_{Y} \cdot \cos (\beta \cdots \alpha)
$$

where $g_{k} \equiv 2 m_{V} / v$ for $V=W$ or $Z$. There are no tree-level couplings of $A$ or $H^{ \pm}$to $I T$. The couplings of $V$ to two neutral Higgs bosons (which must have opposite CP-quantum numbers) are gival by $g_{\phi A Z}\left(p_{\phi}-p_{A}\right)$, where $\phi=h$ or $H$ and the momenta $p_{\phi}$ and $p_{A}$ point into the vertex, and

$$
g_{h A Z}=\frac{y \cos (\beta-\alpha)}{2 \cos \theta_{W}}, \quad g_{H A Z}=\frac{-y \sin (\beta-c)}{2 \cos \theta_{W}}
$$

From the expressions above, we see that the following sum rules must hold separately for $V^{\circ}=H^{\circ}$ and $Z$ :

$$
\begin{aligned}
g_{H V}^{2}+g_{h l \mathrm{l}}^{2} & =g_{1}^{2} m_{1}^{2}, \\
g_{h A Z}^{2}+g_{H A Z}^{2} & =\frac{g^{2}}{4 \cos ^{2} \theta_{W}}, \\
g_{\phi Z Z}^{2}+4 m_{Z}^{2} g_{\phi A Z}^{2} & =\frac{g^{2} m_{Z}^{2}}{\cos ^{2} \theta_{W}} . \quad \dot{\phi}=h, H .
\end{aligned}
$$

Similar considerations also hold for the coupling of $h$ and $H$ to $W^{-} H^{\mp}$. Four-point couplings of vectur bosons and Higgs bosons can be found in ref. [2]. The properties of the three-point and four-point Higgs boson-vector boson couplings are conveniently summarized by listing the couplings that are proportional 
to either $\sin (\beta-\alpha)$ or $\cos (\beta-\alpha)$, and the couplings that are independent of $\alpha$ and $\beta$ [2]:

$$
\begin{array}{lll}
\frac{\cos (\beta-\alpha)}{H W^{+} W^{-}} & \frac{\sin (\beta-\alpha)}{h W^{+} W^{-}} & \text {angle-independent } \\
H Z Z & h Z Z & \\
Z A h & Z A H & Z H^{+} H^{-}, \gamma H^{+} H^{-} \\
W^{ \pm} H^{\mp} h & W^{ \pm} H^{\mp} H & W^{ \pm} H^{\mp} A \\
Z W^{ \pm} H^{\mp} h & Z W^{ \pm} H^{\mp} H & Z W^{ \pm} H^{\mp} A \\
\gamma W^{ \pm} H^{\mp} h & \gamma W^{ \pm} H^{\mp} H & \gamma W^{ \pm} H^{\mp} A \\
\multicolumn{1}{c}{-} & - & V V \phi \phi, V V A A, V V H^{+} H
\end{array}
$$

where $\phi=h$ or $H$ and $V V=W^{+} W^{-}, Z Z, Z \gamma$ or $\gamma \gamma$. Note in particular that all vertices in the theory that contain at least one vector boson and exactly one non-minimal Higgs boson state $\left(H, A\right.$ or $\left.H^{ \pm}\right)$ are proportional to $\cos (\beta-\alpha)$. This can be understood as a consequence of unitarity sum rules which must be satisfied by the tree-level amplitudes of the theory $[24,25,131,132]$.

In the MSSM, the tree-level Higgs couplings to fermions obey the following property: $\Phi_{d}^{0}$ couples exclusively to down-type fermion pairs and $\Phi_{u}^{0}$ couples exclusively to up-type fermion pairs. This pattern of Higgs-fermion couplings defines the Type-II two-Higgs-doublet model [133,2]. The gauge-invariant Type-II Yukawa interactions (using 3rd family notation) are given by:

$$
-\mathcal{L}_{\text {Yukawa }}=h_{t}\left[\bar{t} P_{L} t \Phi_{u}^{0}-\bar{t} P_{L} b \Phi_{u}^{+}\right]+h_{b}\left[\bar{b} P_{L} b \Phi_{d}^{0}-\bar{b} P_{L} t \Phi_{d}^{-}\right]+\text {h.c. }
$$

where $P_{L} \equiv \frac{1}{2}\left(1-\gamma_{5}\right)$ is the left-handed projection operator. [Note that $\left(\bar{\Psi}_{1} P_{L} \Psi_{2}\right)^{\dagger}=\bar{\Psi}_{2} P_{R} \Psi_{1}$, where $P_{R} \equiv \frac{1}{2}\left(1+\gamma_{5}\right)$.] Fermion masses are generated when the neutral Higgs components acquire vacuum expectation values. Inserting eq. (5) into cq. (26) yiclds a relation between the quark masses and the Yukawa couplings:

$$
h_{b}=\frac{\sqrt{2} m_{b}}{v_{d}}=\frac{\sqrt{2} m_{b}}{v \cos \beta}, \quad h_{t}=\frac{\sqrt{2} m_{t}}{v_{u}}=\frac{\sqrt{2} m_{t}}{v \sin \beta} .
$$

Similarly, one can define the Yukawa coupling of the Higgs boson to $\tau$-leptons (the latter is a down-type fermion). The couplings of the physical Higgs bosons to the third generation fermions is obtained from eq. (26) by using eqs. (6)-(8). In particular, the couplings of the neutral Higgs bosons to $f \vec{f}$ relative to the Standard Model value, $g m_{f} / 2 m_{W}$, are given by

$$
\begin{aligned}
h b \bar{b} \quad\left(\text { or } h \tau^{+} \tau^{-}\right): & -\frac{\sin \alpha}{\cos \beta}=\sin (\beta-\alpha)-\tan \beta \cos (\beta-\alpha), \\
h t \bar{t}: & \frac{\cos \alpha}{\sin \beta}=\sin (\beta-\alpha)+\cot \beta \cos (\beta-\alpha),
\end{aligned}
$$




$$
\begin{aligned}
& H b \bar{b} \quad\left(\text { or } H \tau^{+} \tau^{-}\right): \quad \frac{\cos \alpha}{\cos \beta}=\cos (\beta-\alpha)+\tan \beta \sin (\beta)(r) \\
& \left.H+\bar{t}: \quad \frac{\sin a}{\sin \theta}=\cos (\theta-\theta)-\cot \theta \sin \theta+a\right) \\
& \left.A b \bar{b} \text { (or } A \tau^{+} \tau^{-}\right): \quad \gamma \tan \beta \\
& \text { Ht } \bar{t} \text {. } \gamma_{s} \cot \beta \text {, }
\end{aligned}
$$

(the $\gamma_{5}$ indicates a pseudoscalar coupling), and the charged Higgs boson couplings to formion pars. with all particles pointing into the vertex, are given by

$$
\begin{aligned}
g_{H^{-}+\bar{b}} & =\frac{g}{\sqrt{2} m_{W}}\left[m_{+} \cot b P_{R}+m_{b} \tan \theta P_{L}\right] . \\
g_{I^{-} T^{+}{ }^{\prime}} & =\frac{g}{\sqrt{2} m_{W}}\left[m_{\tau} \tan \beta P_{L}\right] .
\end{aligned}
$$

We next examine the behavior of the Higgs couplings at large tan $\beta$. This limit is of particulat interest since at large $\tan \beta$. some of the Higgs couplings to down-type fermions can bo significantly enhanced. ${ }^{11}$ Consider two large $\tan \beta$ regions of interest: (i) If $m_{A} \gg m_{Z}$, then the decoupling limit in rached, in which $|\cos (\beta-\alpha)| \ll 1$ and $m_{n} \simeq m_{1}$. From eqs. (18) (33), it follows that the $b \dot{b} H$ and $b b .4$ couplings have equal strength and are significantly enhanced (by a factor of tan 3 ) relative to the bhh sin coupling, whereas the VVH coupling is negligibly small. In contrast, the values of the $V T h$ and boh couplings are equal to the corresponding couplings of the Standard Model Higgs boson. To show that the value of the $b \bar{b} h$ coupling $[e q .(28)]$ reduces to that of $b \bar{b} h_{\mathrm{SM}}$ in the decoupling limit. note that eq. (18) implies that $|\tan \beta \cos (\beta-\alpha)| \ll 1$ when $m_{, 1} \gg m_{Z}$ even when $\tan \beta \gg 1$. Indeed, $h$ is a SM-lik Higgs boson. (ii) If $m_{A} \lesssim m_{Z}$ and $\tan \beta \gg 1$, then $|\cos (\beta \cdot \alpha)| \sim 1$ [sen fig. 11] and $m_{f_{L}} \simeq m_{4}$. In this casc. the $b \vec{b} h$ and $b \vec{b} A$ couplings have equal strength and are significantly enhanced (by a factor of tan $\beta$ ) relative to the $b \vec{b} h_{\mathrm{SM}}$ coupling, while the $T h$ coupling is negligibly small. Using eq. (22) it follows that the $I T H$ coupling is equal in strength to the $V T_{\text {SW }}$ coupling. In this case. it is conventional to refer to $H$ as a SM-like Higgs boson. However, this nomenclature is somewhat inaccurate. since the value of the $b \bar{b} H$ coupling can differ from the corresponding $b \bar{b} h_{\mathrm{s} S \mathrm{~N}}$ coupling when tan $\beta \gg 1$ [since in case (a) where $|\sin (\beta-\alpha)| \ll 1$, the product tan $\beta \sin (\beta-\gamma)$ need not be particularly small]. Note that in billi cases (i) and $i i)$ above, only two of the three neutral Higgs bosons have enhanced conplings to bit.

The decoupling limit of $m_{1} \gg m_{z}$ is effective for all values of tant? It is edst to check that the pattern of all Higgs couplings displayed in egs. (20) - (33) respect the decoupling limit. That is. in the

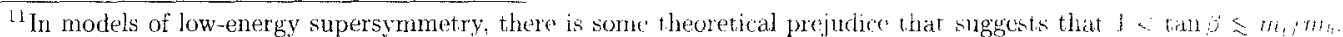
with the fermion ruming masses evaluated at the electroweak scale. For example tan $\beta \leqslant 1\left[\right.$ tan,$\left.b>m_{t} / m_{h}\right]$ is disfavmed

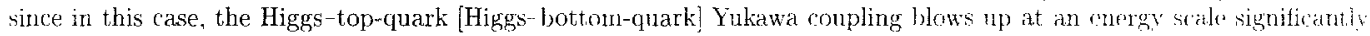
below the Planck scale.
} 

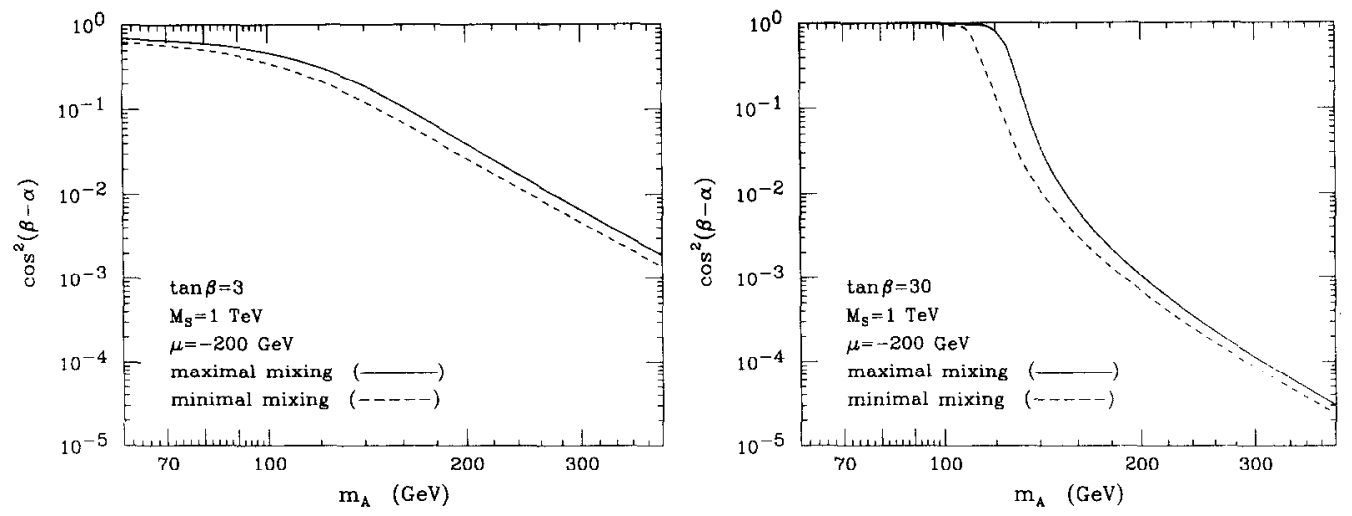

Figure 11: The value of $\cos ^{2}(\beta-\alpha)$ is shown as a function of $m_{A}$ for two choices of $\tan \beta=3$ and $\tan \beta=30$. When radiative-corrections are included, one can define an approximate loop-corrected angle $\alpha$ as a function of $m_{1}$, $\tan \beta$ and the MSSM parameters. In the figures above, we have incorporated radiative corrections, assuming that $M_{\text {SLSY }} \equiv M_{Q}=$ $M_{l}=M_{D}=1 \mathrm{TeV}$. In addition, two extreme cases for the squark mixing parameters are shown (see Sections 3.2 and 3.3 for further discussion of the radiative corrections and their dependence on the supersymmetric parametersi. The decoupling effect expected from eq. (18), in which $\cos ^{2}(\beta-\alpha) \propto m_{Z}^{4} / m_{A}^{4}$ for $m_{A} \gg m_{Z}$, continues to hold even when radiative corrections are included.

limit where $m_{A} \gg m_{Z}, \cos (\beta-\alpha)=\mathcal{O}\left(m_{Z}^{2} / m_{A}^{2}\right)$, which means that the $h$ couplings to Standard Model particles approach values corresponding precisely to the couplings of the SM Higgs boson. There is a significant region of MSSM Higgs sector parameter space in which the decoupling limit applies, because $\cos (\beta-\alpha)$ approaches zero quite rapidly once $m_{A}$ is larger than about $200 \mathrm{GeV}$, as shown in fig. 11 . As a result, over a significant region of the MSSM parameter space, the search for the lightest CP-even Higgs boson of the MSSM is equivalent to the search for the Standard Model Higgs boson. This result is more general; in many theories of non-minimal Higgs sectors, there is a significant portion of the parameter space that approximates the decoupling limit. Consequently, simulations of the Standard Model Higgs signal are also relevant for exploring the more general Higgs sector.

\subsection{Radiatively-Corrected MSSM Higgs Masses}

The discussion of Section 3.1 was based on a tree-level analysis of the Higgs sector. However, radiative corrections can have a significant impact on the predicted values of Higgs masses and couplings. The radiative corrections involve both loops of Standard Model particles and loops of supersymmetric part- 
ners. The dominant effects arise from loops involving the third generation quarks and squarks and are proportional to the corresponding Yukawa couplings. Thus, we first review the parameters that cuntrol the masses and mixing of the third-generation squarks. (We shall neglect intergenerational mixing effects, which have little impact on the discussion that follows.)

For each left-handed and right-handed quark of fixed flavor, $q$, there is a corresponding supersymmetric partner $\tilde{q}_{L}$ and $\tilde{q}_{R}$, respectively. These are the so-called interaction eigenstates, which mix according to the squark squared-mass matrix. The mixing angle that diagonalizes the squark mass matrix will be denoted by $\theta_{\tilde{q}}$. The squark mass eigenstates, denoted by $\widetilde{q}_{1}$ and $\widetilde{q}_{2}$, are obtained by diagonalizing the following $2 \times 2$ matrix

$$
\left(\begin{array}{cc}
M_{Q}^{2}+m_{f}^{2}+D_{t} & m_{f} X_{f} \\
m_{j} X_{f} & M_{R}^{2}+m_{f}^{2}+D_{H}
\end{array}\right):
$$

where $D_{L} \equiv\left(T_{3 f}-e_{f} \sin ^{2} \theta_{W}\right) m_{Z}^{2} \cos 2 \beta$ and $D_{R} \equiv e_{f} \sin ^{2} \theta_{W} m_{Z}^{2} \cos 2 \beta$. In addition, $f=t, M_{k} \equiv M_{l}$. $e_{t}=2 / 3$ and $T_{3 f}=1 / 2$ for the top-squark squared-mass matrix. and $f=b, M_{R} \equiv M_{D}: c^{\prime} b=-1 / 3$ and $T_{3 f}=-1 / 2$ for the bottom-squark squared-mass matrix. The squark mixing parameters are given by

$$
X_{t} \equiv A_{t}-\mu \cot B, \quad \quad X_{b} \equiv A_{b}-\mu \tan B .
$$

Thus, the top-squark and bottom-squark masses and mixing angles depend on the supersymmerin: Higgsino mass parameter $\mu$ and the soft-supersymmetry-breaking parameters: $M_{Q}, M_{L} \cdot M_{D}, A_{t}$ and $A_{b}$. For simplicity, we shall initially assume that $A_{t}, A_{b}$ and $\mu$ are real parameters. That is, we negled possible CP-violating effects that can enter the MSSM Higgs sector via radiative corrections. The impact on new MSSM sources of CP-violation on the Higgs sector will be addressed in Section 3.2.3.

\subsubsection{Radiatively-corrected Higgs masses in the CP-conserving MSSM}

The radiative corrections to the Higgs squared-masses have been computed by a number of techniques, and using a variety of approximations such as the effective potential at one-loop [134-136] and twoloops [137 140], and diagrammatic methods [141-146]. Complete one-loop diagrammatic computations of the MSSM Higgs masses have been presented by a number of groups [143,144]; and partial two-loop; diagrammatic results are also known $[145,146]$. These include the $\mathcal{O}\left(m_{t}^{2} h_{f}^{2} \alpha_{s}\right)$ contributions 10 the neutral CP-even Higgs boson squared-masses in the on-shell scheme [146]. Finally. renormalization group methods (to be discussed further below) provide a powerful technique for identifying many uf the most important contributions to the radiatively corrected Higgs masses [147-150]. Typical results for the radiatively corrected value of $m_{h}$ as a function of the relevant supersymmetrit: parameters are shown in fig. 12.

One of the most striking effects of the radiative corrections to the MSSM Higgs sector is the modification of the upper bound of the light CP-even Higgs mass, as first noted in refs. [134] and [111. Consider the region of parameter space where $\tan \beta$ is large and $m_{+} \gg m_{z}$. In this limit, the tree-freel 

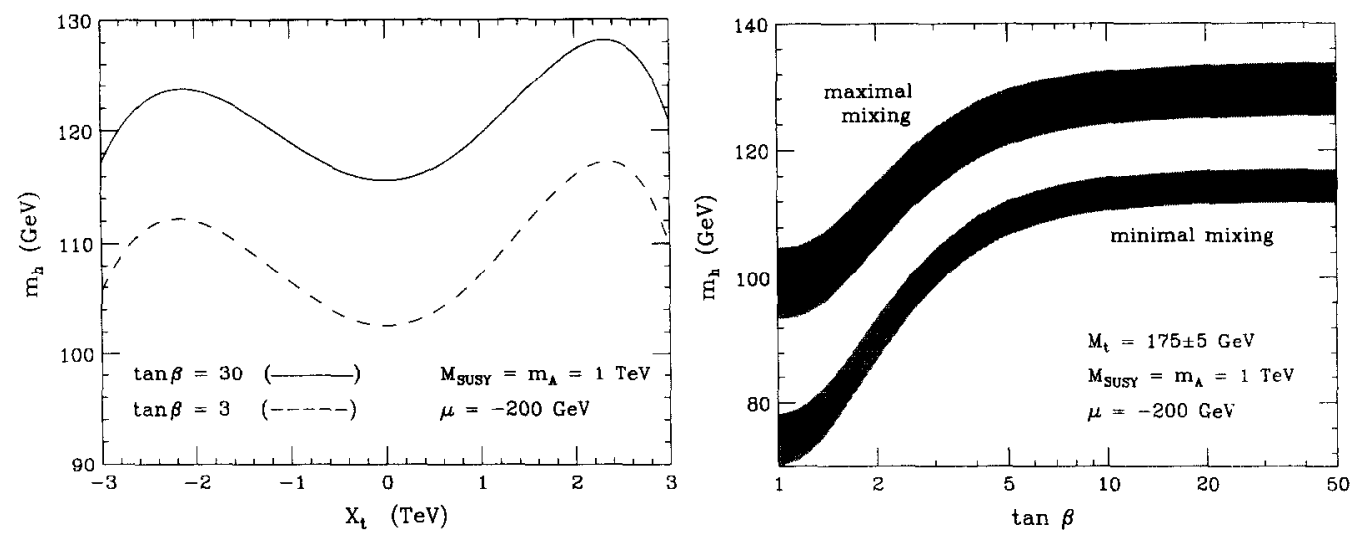

Figure 12: The radiatively corrected light CP-even Higgs mass is plotted (a) as a function of $X_{t}$, where $X_{t} \equiv A_{t}-\mu$ cot $\beta$, for $M_{t}=174.3 \mathrm{GeV}$ and two choices of $\tan \beta=3$ and 30 , and (b) as a function of $\tan \beta$, for the maximal mixing [upper band] and minimal mixing [lower band] benchmark cases. In (b), the central value of the shaded bands corresponds to $M_{t}=175 \mathrm{GeV}$, while the upper [lower] edge of the bands correspond to increasing [decreasing] $M_{t}$ by $5 \mathrm{GeV}$. In both (a) and (b), $m_{A}=1 \mathrm{TeV}$ and the diagonal soft squark squared-masses are assumed to be degenerate: $M_{\mathrm{SUSY}} \equiv M_{Q}=$ $M_{U}-M_{L}-1 \mathrm{TeV}$.

prediction for $m_{h}$ corresponds to its theoretical upper bound, $m_{h}=m_{Z}$. Including radiative corrections, the theoretical upper bound is increased. The dominant effect arises from an incomplete cancellation ${ }^{12}$ of the top-quark and top-squark loops (these effects cancel in the cxact supcrsymmetric limit). The qualitative behavior of the radiative corrections can be most easily seen in the large top squark mass limit, where in addition, the splitting of the two diagonal entries and the off-diagonal entry of the top-squark squared-mass matrix are both small in comparison to the average of the two top-squark squared-masses:

$$
M_{\mathrm{S}}^{2} \equiv \frac{1}{2}\left(M_{i_{1}}^{2}+M_{T_{2}}^{2}\right) .
$$

In this case, the upper bound on the lightest CP-even Higgs mass is approximately given by

$$
m_{h}^{2} \lesssim m_{Z}^{2}+\frac{3 g^{2} m_{t}^{4}}{8 \pi^{2} m_{\mathrm{W}}^{2}}\left[\ln \left(\frac{M_{S}^{2}}{m_{t}^{2}}\right)+\frac{X_{t}^{2}}{M_{S}^{2}}\left(1-\frac{X_{t}^{2}}{12 M_{S}^{2}}\right)\right]
$$

\footnotetext{
${ }^{12}$ In certain regions of parameter space (corresponding to large $\tan \beta$ and large values of $\mu$ ), the incomplete cancellation of the bottom-quark and bottom-squark loops can be as important as the corresponding top sector contributions. For simplicity, we ignore this contribution in eq. (39).
} 
The more complete treatments of the radiative corrections cited above show that eq. (39) somewhat. uverestimates the true upper bound of $m_{h}$. Nevertheless, eq. (39) correctly reflects some noteworthy features of the more precise result. First, the increase of the light (P-even Higgs mass bound beyond $m_{Z}$ can be significant. This is a consequence of the $m_{1}^{1}$ enhancement of the mis-luop radiative correction Second, the dependence of the light Higgs mass on the top squark mixing parameter $\mu_{t}$ implies that (for a given value of $M_{\mathrm{S}}$ ) the upper bound of the light Higgs mass initially increases with $X_{t}$ and reaches its maximal value for $X_{t}=\sqrt{6} M_{\mathrm{S}}$. This point is referred to as the muximal mixing case (whereas $\mathrm{X}_{t}=0$ corresponds to the minimal mixing case). In a more complete computation that includes both two-loop logarithmic and non-logarithmic corrections, the $I_{f}$ values corresponding to maximal and minimal mixing are shifted and exhibit an asymmetry under $X_{t} \rightarrow-X_{t}$ as shown in fig. 1? In thr numerical analysis presented in this and subsequent figures in this section. we assume for simplirity that the third generation diagonal soft-supersymmetry-breaking squark squared-masses are degenerate: $M_{\mathrm{SUSY}} \equiv M_{Q}=M_{U^{\prime}}=M_{D}$, which defines the parameter $M_{\mathrm{SI} S Y},{ }^{1: 3}$

Third, note the logarithmic sensitivity to the top squark masses. Naluralness arguments that ulderlie low-energy supersymmetry imply that the supersymmetric particle masses should not bie larger than a few TeV. Still, the precise upper bound on the light Higgs mass depends on the specific choice for the upper limit of the top-squark masses. The dependence of the light Higgs mass oftained by the more complete computation as a function of $M_{\mathrm{SI} S \mathrm{~S}}$ is shown in fig. $733^{1.1}$

As noted above, the largest contribution to the one-loop radiative corrections is enllarred by a furfor of $m_{t}^{4}$ and grows logarithmically with the top squark mass. Thus, higher order radiative corrections 'anl be non-negligible for large top squark masses, in which case the large logarithms must be resunmod. Renormalization group (RG) techniques for resumming the leading logarithms have been dereloped by a number of authors $\left[\begin{array}{ll}147 & 149\end{array}\right]$. The computation of the $\mathrm{RG}$-improved one-loop (orrections requires numerical integration of a coupled set of RG equations [148]. Although this procedure has been carrict out in the literature, the analysis is unwieldy and not easily amenable to large-scale Monte-Carlo studirs. It turns out that over most of the parameter range. it is sufficient to include the leading and sub-leading logarithms at two-loop order. (Some additional non-logarithmic terns, which cannot be ascertained by the renormalization group method, must also be included [151].) (ompact analytic expressions have been obtained for the dominant one and two-loop coutsibutions to the matrix elements of the radiatively-corrected CP-cven Higgs squared-mass matrix:

$$
\mathcal{M}^{2} \equiv\left(\begin{array}{ll}
\mathcal{M}_{11}^{2} & \mathcal{M}_{12}^{2} \\
\mathcal{M}_{12}^{2} & \mathcal{M}_{12}^{2}
\end{array}\right)=\mathcal{M}_{11}^{2}+i \mathcal{M}^{2}
$$

where the tree-level contribution $\mathcal{M}_{0}^{2}$ was given in eq. (11) and $\delta \mathcal{M}^{2}$ is the contribution from the

\footnotetext{
${ }^{13}$ We also assume that $M_{\text {SUSY }} \gg m_{t}$, in which case it follows that $M_{S}^{2} \simeq M_{\text {SLSY }}^{2}$ up to corrections of $\mathcal{O}\left(m_{t}^{2} / M_{S[S Y}^{2}\right.$.

${ }^{14}$ The flattening of the curves in fig. 13 as a function of $M_{\text {SUSY }}$ in the maximal mixing scenario is due to the squarkmixing contributions at two-lonps which partially cancel the contributions that grow lngarithmically with $M_{\mathrm{SESY}}$.
} 

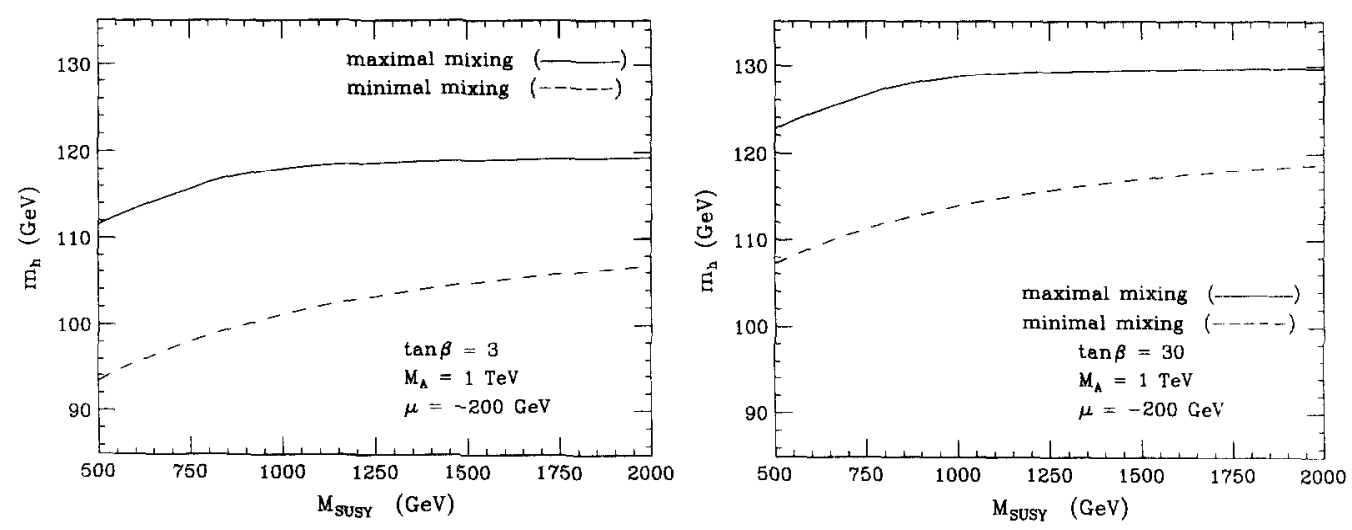

Figure 13: The radiatively corrected light CP-even Higgs mass is plotted as a function of $M_{\mathrm{SUSY}} \equiv M_{Q}=M_{U}=M_{D}$, for $M_{t}=174.3 \mathrm{GeV}, m_{A}=1 \mathrm{TeV}$ and two choices of $\tan \beta=3$ and $\tan \beta=30$. Maximal mixing and minimal mixing are defined according to the value of $X_{t}$ that yields the maximal and minimal Higgs mass as shown in fig. 12(a).

radiative corrections. The dominant corrections to $\mathcal{M}^{2}$, coming from the one-loop top and bottom quark and top and bottom squark contributions plus the two-loop leading logarithmic contributions, are given to $\mathcal{O}\left(h_{t}^{4}, h_{b}^{4}\right)$ by $[149,150,152]$

$$
\begin{aligned}
\delta \mathcal{M}_{11}^{2} \simeq & -\bar{\mu}^{2} x_{t}^{2} \frac{h_{t}^{4} v^{2}}{32 \pi^{2}} s_{\beta}^{2}\left[1+c_{11} \ln \left(\frac{M_{S}^{2}}{m_{t}^{2}}\right)\right]-\bar{\mu}^{2} a_{b}^{2} \frac{h_{b}^{4} v^{2}}{32 \pi^{2}} s_{\beta}^{2}\left[1+c_{12} \ln \left(\frac{M_{S}^{2}}{m_{t}^{2}}\right)\right], \\
\delta \mathcal{M}_{12}^{2} \simeq & -\bar{\mu} x_{t} \frac{h_{t}^{4} v^{2}}{32 \pi^{2}}\left(6-x_{t} a_{t}\right) s_{\beta}^{2}\left[1+c_{31} \ln \left(\frac{M_{S}^{2}}{m_{t}^{2}}\right)\right]+\bar{\mu}^{3} a_{b} \frac{h_{b}^{4} v^{2}}{32 \pi^{2}} s_{\beta}^{2}\left[1+c_{32} \ln \left(\frac{M_{S}^{2}}{m_{t}^{2}}\right)\right], \\
\delta \mathcal{M}_{22}^{2} \simeq & \frac{3 h_{t}^{4} v^{2}}{8 \pi^{2}} s_{\beta}^{2} \ln \left(\frac{M_{S}^{2}}{m_{t}^{2}}\right)\left[1+\frac{1}{2} c_{21} \ln \left(\frac{M_{S}^{2}}{m_{t}^{2}}\right)\right] \\
& +\frac{h_{t}^{4} v^{2}}{32 \pi^{2}} s_{\beta}^{2} x_{t} a_{t}\left(12-x_{t} a_{t}\right)\left[1+c_{21} \ln \left(\frac{M_{S}^{2}}{m_{t}^{2}}\right)\right]-\bar{\mu}^{4} \frac{h_{b}^{4} v^{2}}{32 \pi^{2}} s_{\beta}^{2}\left[1+c_{22} \ln \left(\frac{M_{S}^{2}}{m_{t}^{2}}\right)\right],
\end{aligned}
$$

where $s_{B} \equiv \sin \beta, c_{\beta} \equiv \cos \beta$, and the coefficients $c_{i j}$ are:

$$
c_{i j} \equiv \frac{t_{i j} h_{t}^{2}+b_{i j} h_{b}^{2}-32 g_{3}^{2}}{32 \pi^{2}}
$$


$\left(t_{11}, t_{12}, t_{21}, t_{22}, t_{31}, t_{32}\right)=(12,-4,6,-10,9,-7)$ and $\left(b_{11}, b_{12}, b_{21}, b_{22}, b_{31}, b_{32}\right)=(\ldots 4,12.2 .18 . \cdot 1.151$ Above, $h_{t}$ and $h_{0}$ are the top and bottom quark Yukawa couplings [see eqs. (55) (57)], 93 is the strong QCD coupling, $v=246 \mathrm{GeV}$ is the SM Higgs vacum expectation value. and $M_{S}^{2}-\frac{1}{2}\left(M_{\tilde{i}_{1}}^{2}+M_{\tilde{i}_{2}}^{\prime}\right)$ is the average squared top squark mass. ${ }^{15}$ The $\delta \mathcal{M}_{i j}^{2}$ also depend on the MSSW parameters $A_{f} A_{b}$ and $\mu$ that enter the off-diagonal top-squark and bottom-squark squarcd-mass matrices. We rmploy the following notation: $\bar{\mu} \equiv \mu / M_{S}: a_{t} \equiv A_{1} / M_{S}, a_{b} \equiv A_{b} / M_{S}$ and $x_{t} \equiv X_{1} / M_{4}$. where $X_{t} \equiv A_{1}-\mu(0)$, Diagonalizing the CP-even Higgs squared-mass matrix vields radiatjvely-correted valuen for min. min and the mixing angle $x{ }^{16}$ The end result is a prediction for the Higgs mass in terms of running parameters in the $\overline{\mathrm{MS}}$ scheme. It is a simple matter to relate these parameters to the corrosponding on-shell parameters used in the diagrammatic: calculations [138.151].

Additional non-logarithmic two-loop contributions. which can gene'vate a non-negligible shith in the Higgs mass (of a few GeV). must also be included. "A compact analytical expression that incorpurates these effects at $\mathcal{O}\left(m_{t}^{2} h_{t}^{2} \alpha_{s}\right)$ was given in ref. [154] (with further refinements provided bx ref. [15j] to $1 \mathrm{atk} \boldsymbol{k}^{2}$ into account the possibility of arbitrary top-squark splitting), and the corresponding conertions propurtional to $h_{b}^{2} \alpha_{s}$ can be found in ref. [140]. An important soures of such contributions are the one-loop supersymmetric threshold corrections to the relation between the Higgs-top-cuark and Higgs-bottonquark Yukawa couplings and the corresponding quark masses [eqs. (j6) and (57)]. These generate n Iun-logarithmic two-loop shift of the radiatively corrected Higgs matsis pruportional to the corresponding squark mixing parameters. One consequence of these contributions [151] is the atsymeng in flus

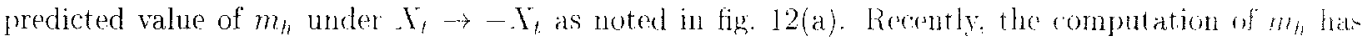
been further refined by the inclusion of genuine two-loop corrections of $\mathcal{O}\left(m_{l}^{2} h_{l}^{4}\right)[139$. and estinnates of the two-loop corrections proportional to $h_{b}^{2} h_{t}^{2}$ and $h_{b}^{4}[140]$ (which can be numerically relevant for values of $\left.\tan \beta \gtrsim m_{t} / m_{t}\right)$. These non-logarithmic corrections. which depend on the third gencration squark mixing parameters, can slightly increase the value of the ratiatively-currected Higgs mass

The numerical results displayed in figs. $11 \ldots 14$ are based on the alculations of refs. $[149]$ and $[150$. with improvements as described in refs. $[146]$ and [151]. The supersymuctric parameters in the maximal and minimal mixing cases have been chosen according to the first two benchmark sonarios if wef. $\mid 150 \mathrm{j}$. of particular interest is the upper bound for the lightest CP-even Higgs mass (mh). At fixed tan 1 . 1 . maximal value of $m_{h}$ is reached for $m_{1} \gg m_{z}$ (see fig. 14). Taking $m_{1}$ large. fig. 12(b) illustrates that. the: maximal value of the lightest CP-even Higgs mass bound is redized at large tan 3 in the ase we

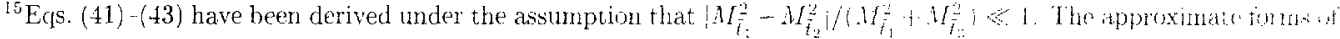

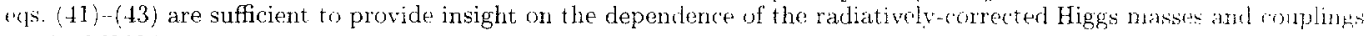
on the MSSM parameters. although our numerical work is based on nore exart forms for thesere expressions

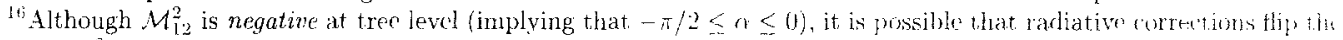
sign of $\mathcal{M}_{12}^{q}$. Thus, the range of the radiatively corrected angle of can be taken on bet $-\pi / 2 \leq 0 \leq \pi / 2$.

${ }_{17}$ An improved procedure for computing the radiatively-corrected neutral Higgs mass matrix and the charged Higgs thass in a self-consistent way (including possible CP-violating effects), which incorporates one-locp supersymmetric threshold corrections to the Higgs-top-quark and Higgs-bottom-quark Yukawa colplings. an be fomme in ref. 1.53 .
} 


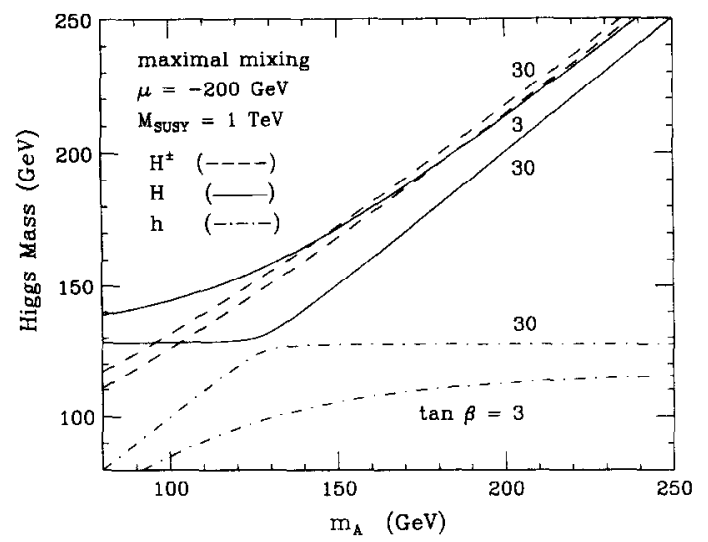

Figure 14: Lightest CP-even Higgs mass $\left(m_{h}\right)$, heaviest CP-even Higgs mass $\left(m_{H}\right)$ and charged Higgs mass $\left(m_{H^{ \pm}}\right)$as a function of $m_{A}$ for two choices of $\tan \beta=3$ and $\tan \beta=30$. Here, we have taken $M_{t}=174.3 \mathrm{GeV}$, and we have assumed that the diagonal soft squark squared-masses are degenerate: $M_{\mathrm{SUSY}} \equiv M_{Q}=M_{U}=M_{D}=1 \mathrm{TeV}$. In addition, we choose the other supersymmetric parameters corresponding to the maximal mixing scenario. The slight increase in the charged Higgs mass as $\tan \beta$ is increased from 3 to 30 is a consequence of the radiative corrections.

maximal mixing. For each value of $\tan \beta$, we denote the maximum value of $m_{h}$ by $m_{h}^{\max }(\tan \beta$ ) [this value also depends on the third-generation squark mixing parameters]. Allowing for the uncertainty in the measured value of $m_{t}$ and the uncertainty inherent in the theoretical analysis, one finds for $M_{\text {SUSY }} \lesssim 2 \mathrm{TeV}$ that $m_{h} \leq m_{h}^{\max } \equiv m_{h}^{\max }(\tan \beta \gg 1)$, where

$$
\begin{aligned}
& m_{h}^{\max } \simeq 122 \mathrm{GeV}, \quad \text { if top-squark mixing is minimal, } \\
& m_{h}^{\max } \simeq 135 \mathrm{GeV}, \quad \text { if top-squark mixing is maximal } .
\end{aligned}
$$

In practice, parameters leading to maximal mixing are not expected in typical models of supersymmetry breaking. Thus, in general, the upper bound on the lightest Higgs boson mass is expected to be somewhere between the two extreme limits quoted above. Cross-checks among various programs [157] and rough estimates of higher order corrections not yet computed suggest that the results for Higgs masses should be accurate to within about 2 to $3 \mathrm{GeV}$ over the parameter ranges displayed in figs. 12-14.

In fig. 14, we exhibit the masses of the CP-even neutral and the charged Higgs masses as a function 
of $m_{A}$. The squared-masses of the lighter and heavier neutral CP-even Higgs ares related $)^{14}$

$$
m_{H}^{2} \cos ^{2}(\beta-\alpha)+m_{h}^{2} \sin ^{2}(\beta-\alpha)=\left[m_{h}^{\max }(1 \cdot a u, \beta)\right]^{2}
$$

It is interesting to consider the behavior of the CP-even Higgs masses in the large tan of reginte. Fon large values of $\tan \beta$ and for $m_{A} / \tan \beta \ll m_{h}^{\max }(\tan \beta)$, the off-diagonal elements of the Higgs squared-1nats matrix $\mathcal{M}^{2}$ become small compared to the diagonal clements $\left|\mathcal{M}_{12}^{2}\right| \ll \mathcal{M}_{11}^{2}-\mathcal{M}_{22}^{2}: \mathcal{M}_{12}^{4} \ll \mathcal{M}_{1}^{2} \mathcal{M}_{22}^{2}$. Hence the two CP-cven Higgs squared-masses are approximately given by the diagonal nements of $\mathcal{M}^{2}$ As above, we employ the notation where $m_{h}^{\text {max }}$ refers to the asymptotic value of $m_{h}$ at large tan "s and $m_{A}$ the actual numerical value of $m_{h}^{\max }$ depends primarily on the assumed ralues of the thind generation squark mass and mixing parameters). If $m_{A}>m_{1}^{\text {plax }}$, then $m_{h}=m_{h}^{\text {max }}$ and $m_{n}=m_{\text {! }}$. whereas if $m_{1}<m_{h}^{\max }$. then $m_{h} \simeq m_{1}$ and $m_{H} \simeq m_{h}^{\max }$. This behavior can besen in fig. 11 .

\subsubsection{MSSM Higgs mass limits after LEP}

Xo significant evidence for a Higgs signal has been detected at LEP [161]. Ait a result one can obtain bounds on the possible MSSM Higgs parameters. These limits are often displayed in the $m_{A}$ tan 8 plane, although there is additional dependence on various MSSM parameters that effect the radiative corrections to the Higgs masses as discussed above. In representative scans of the MSSM parameters, the LEP Higgs Working Group [161] finds that $m_{h_{h}}>91.0 \mathrm{GeV}$ and $m_{A}>91.9 \mathrm{GeV}$ at $95 \% \mathrm{CL}$. These limits actually correspond to the large $\tan 3$ region in which $Z h$ production is suppressed. as shown in fig. 15 . In this case, the quoted Higgs limits arise as a result of the mon-olservation of $h .4$ and $H .1$ production. As $\tan \theta$ is lowered. the limits on $m_{h}$ and $m_{A}$ become more stringent. In this rexime. the $h .4$ production is suppressed while the $Z h$ production rate approaches its $S \mathrm{SM}$ value. I'hus. in this (ase, the SM Higgs limit applies $\left(m_{h} \gtrsim 114 \mathrm{GeV}\right)$ as shown in fig. $15(a)$. The precise region of MSSM Higgs parameter space that is excluded depends on the values of the MSSM parameters that rontrol the. Higgs mass radiative corrections. For example, a conservative exclusion limit is obtained in the maximal mixing scenario, since in this case the predicted value of $m_{h}$ as a function of $m_{A}$ and tan $\%$ is maximal (with respect to changes in the other MSSM parameters). The excluded regions of the MSSM Higgs parameter space based on the maximal mixing benchmark scenario of ref. [156], are shown in fig. 15. and correspond to the exclusion of the range $0.5<\tan \beta<2.4$ at the $9.9 \%$ CL. However be tan exclusion region can still be significantly reffued (even to the point of allowing all tan 8 values) by a.f. taking $M_{\text {SUSY }}=2 \mathrm{TeV}$ and $m_{t}=180 \mathrm{GeV}$ (which still lies within the error bars of the experimentally neasured value), and allowing for the theoretical uncertainty in the prediction of $m_{h}^{\text {trax }}[162]$

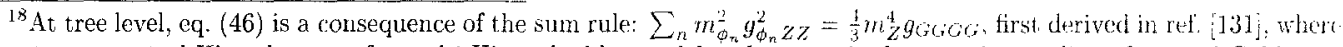
the $\phi_{n}$ are neutral Higgs bosons of a multi-Higgs-doublet model and $g G G G G$ is the quartir coupling of ne!utral Goldstont bosons. (In the MSSM, $g_{G G G G}=\frac{3}{4} g^{2} \cos ^{2} 2 \beta / \cos ^{2} \theta_{W}$ [2].) A general discussion of related trec-level Higgs mass sum jules and bounds can be found in ref [158]. In fact, e'(1. (46) is more general and applies to the radiatively-correcter? MSSM Higgs sector [159.160] in the approximation where the renormalized $\alpha$ in determined as discussed in section 3.3 .1
} 

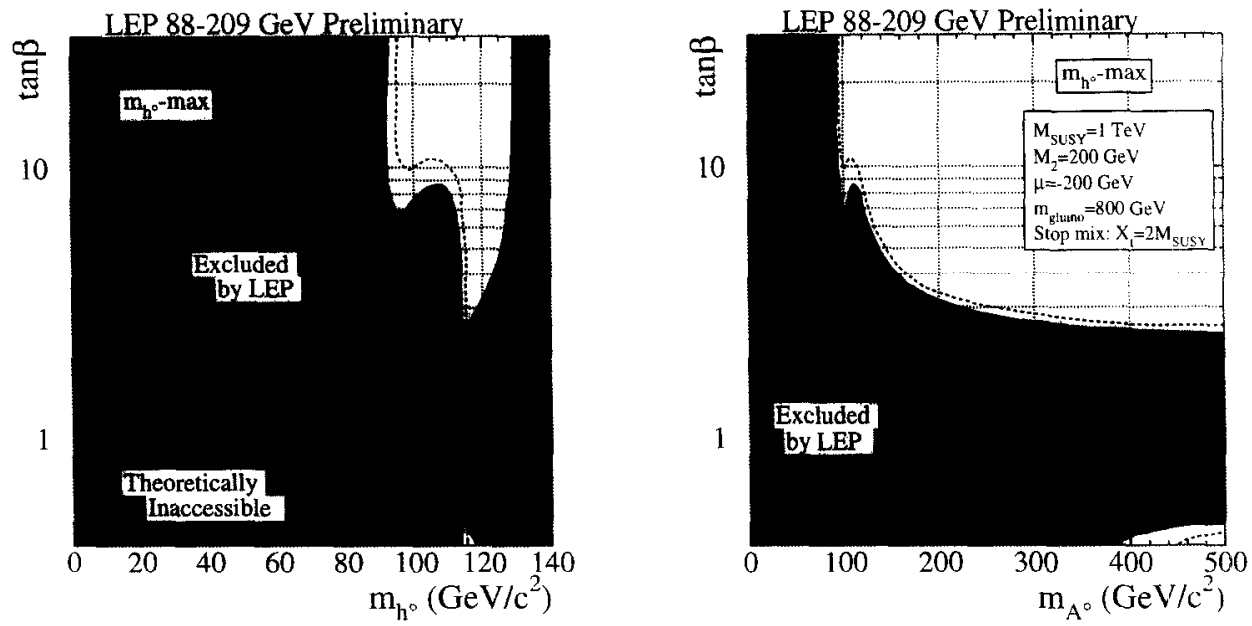

Figure 15: LEP2 contours of the $95 \%$ CL exclusion limits for MSSM Higgs sector parameters as a function of tan $\beta$ and (a) $m_{h}$ and (b) $m_{A}$ (in GeV), taken from ref. [161]. The contours shown have been obtained for MSSM Higgs parameters chosen according to the maximal mixing benchmark of ref. [156].

No evidence for the charged Higgs boson has yet been found. The LEP Higgs Working Group quotes a limit of $m_{H^{ \pm}}>78.6 \mathrm{GeV}$ at $95 \% \mathrm{CL}$ [163], which holds for a more general non-supersymmetric twoHiggs doublet model and assumes only that the $\mathrm{H}^{+}$decays dominantly into ${ }^{+}{ }_{\nu}$ and/or $c \bar{s}$. Although the MSSM tree-level bound $m_{H^{ \pm}} \geq m_{W}$ can be relaxed somewhat by radiative corrections, the LEP bound quoted above provides no useful additional constraints on the MSSM Higgs sector.

\subsubsection{Effect of explicit CP-violation on the radiatively-corrected MSSM Higgs masses}

In the Standard Model, CP-violation is due to the existence of phases in the Yukawa couplings of the quarks to the Higgs field, which results in one non-trivial phase in the CKM mixing matrix. In the MSSM, there are additional sources of CP-violation, due to phases in the various supersymmetric mass parameters. In particular, the gaugino mass parameters $\left(M_{i}, i=1,2,3\right)$, the Higgsino mass paraneter, $\mu$. the bilinear Higgs squared-mass parameter, $m_{12}^{2}$, and the trilinear couplings of the squark and slepton fields $(\tilde{f})$ to the Higgs fields, $A_{f}$, may carry non-trivial phases. The existence of these CP phases can 
significantly affect the MSSM Higgs sector through one-loop radiative corrections [164-166,153].

Note that if one sets $\mu=M_{i}=A_{f}=m_{12}^{2}$, then the MSSM Lagrangian possesses two independent global $U(1)$ symmetries - a Peccei-Quinn (PQ) symmetry and an $R$ symmetry. (The quantum numbers of the MSSM fields with respect to $U(1)_{P Q}$ and $U(1)_{R}$ can be found in refs. [167] and [166].) Conscquently, in the MSSM with nonzero values for the above parameters, there are two independent phase redefinitions of the fields that can be used to remove two phases from $\mu, M_{i}, A_{f}$ and $m_{12}^{2}$. However. certain combinations of these parameters remain invariant under such phase redefinitions. The simplest way to determine these combinations is to treat the aforementioned parameters as spurions with quantum numbers under the $\mathrm{U}(1)_{\mathrm{PQ}}$ and $\mathrm{U}(1)_{\mathrm{R}}$ symmetries chosen such that the full MSSM Lagrangian is invariant. One can then easily check that the phases of the parameter combinations, $\arg \left[\mu A_{f}\left(m_{12}^{2}\right)^{*}\right)$ and $\arg \left[\mu M_{i}\left(m_{12}^{2}\right)^{*}\right]$, are indeed invariant under the $\mathrm{U}(1)_{\mathrm{PQ}}$ and $\mathrm{U}(1)_{\mathrm{R}}$ phase redefinitions of the MSSM fields $[167,166]$. Therefore, if one of these two quantities is different from zero (modulo $\pi$ ), one should expect new CP-violating effects induced by the production or exchange of supersymmetric particles.

We have already noted that the tree-level Higgs sector is CP-conserving. This is a consequerce of the fart that $m_{12}^{2}$ is the only possible complex parameter that appears in the tree-level Higgs potential Thus the phase of $m_{12}^{2}$ can be rotated away by redefining the phases of the complex Higgs dontleter appearing in the Lagrangian. The same field redefinition implies that ont ran choose the vacuun expectation values of the two Higgs fields to be real and positive. However, at the one loop-lewel. the Higgs potential acquires a dependence on the parameters $\mu A_{t}$ and $\mu M_{i}$ through loops of third generation squarks and weak gauginos, respectively, which induce non-trivial CP-violating effects. The most important of these CP-violating effects is the generation of mixing between the neutral CP-odd and CP-even Higgs boson states. Therefore, the physical neutral Higgs bosons are no longer CP-eigenstates and the CP-odd Higgs boson mass $\tau_{A}$ is no longer a physical parameier. The dlarged Higgs mass is still physical and can be used as an input for the computation of the neutral Higgs spectrum of the theory [153]. The Higgs mass spectrum can therefore be quite different from the CP-conserving cabse. For example, a large splitting between the masses of the next-to-lightest and the heaviest neutral Higgs bosons is possible if the charged Higgs boson is not too heavy.

For large values of the charged Higgs mass, the decoupling limit applies, and the properties of the lightest neutral Higgs boson state approach those of the SM Higgs boson. That is, for $m_{H}=\gg m_{11}$. the lightest neutral Higgs boson is approximately a CP-even state, with CP-violating couplings that are suppressed by terms of $\mathcal{O}\left(m_{W}^{2} / m_{H^{ \pm}}^{2}\right)$ [168]. In particular, the upper-bound on the lightest nentral Higgs boson mass, which is reached in the decoupling limit, takes the same value as in the CP-conserving case [166]. Nevertheless, there still can be significant mixing between the two heavier neutral mast eigenstates. Quantitatively, the leading contribution to the squared-mass terms that mix CP-even inıI CP-odd eigenstates, $M_{S P}^{2}$ (in a convention where $m_{12}^{2}$ is real) is of order

$$
M_{S P}^{2} \simeq \frac{3 g^{2} m_{t}^{4}\left|\mu-A_{t}\right|}{64 \pi^{2} m_{W}^{2} M_{S}^{2}} \sin \left(\arg \left[\mu A_{t}\right]\right)
$$


Under the reasonable assumption that $\left|\mu A_{t}\right|<10 M_{S}^{2}$, it is clear that the mixing effects between the lightest neutral Higgs boson and the heavier Higgs states are small if the masses of the heavy Higgs bosons are larger than $2 m_{t}$. In this limit, the two heavier states are highly degenerate in mass, and the CP-violating effects may still lead to non-trivial mixing of the two heavier CP-eigenstates. For a detailed study of the Higgs mass spectrum and parametric dependence of the Higgs mass radiative corrections, see ref. [153].

\subsection{Radiatively-Corrected MSSM Higgs couplings}

\subsubsection{Renormalization of $\cos (\beta-\alpha)$}

Radiative corrections also significantly modify the tree-level values of the Higgs boson couplings to fermion pairs and to vector boson pairs. As discussed in Section 3.1, the tree-level Higgs couplings depend crucially on the value of $\cos (\beta-\alpha)$. In first approximation, when radiative corrections of the Higgs squared-mass matrix are computed, the diagonalizing angle $\alpha$ is shifted from its tree-level value. Thus, one may compute a "radiatively-corrected" value for $\cos (\beta-\alpha)$. This provides one important suurce of the radiative corrections of the Higgs couplings. In fig. 11, we show the effect of radiative corrections on the value of $\cos (\beta-\alpha)$ as a function of $m_{A}$ for different values of the squark mixing parameters and $\tan \beta$. One can then simply insert the radiatively corrected value of $\alpha$ into eqs. (20), (21) and (28) -(33) to obtain radiatively-improved couplings of Higgs bosons to vector bosons and to fermions.

The mixing angle $\alpha$ which diagonalizes the mass matrix in eq. (40) can be expressed as:

$$
s_{\alpha} c_{\alpha}=\frac{\mathcal{M}_{12}^{2}}{\sqrt{\left(\operatorname{Tr} \mathcal{M}^{2}\right)^{2}-4 \operatorname{det} \mathcal{M}^{2}}}, \quad c_{\alpha}^{2}-s_{\alpha}^{2}=\frac{\mathcal{M}_{11}^{2}-\mathcal{M}_{22}^{2}}{\sqrt{\left(\operatorname{Tr} \mathcal{M}^{2}\right)^{2}-4 \operatorname{det} \mathcal{M}^{2}}},
$$

where $s_{\alpha} \equiv \sin \alpha$ and $c_{\alpha} \equiv \cos \alpha$. Note that if $\mathcal{M}_{12}^{2} \rightarrow 0$, then either $\sin \alpha \rightarrow 0$ (if $\mathcal{M}_{11}^{2}>\mathcal{M}_{22}^{2}$ ) or $\cos \alpha \rightarrow 0$ (if $\mathcal{M}_{11}^{2}<\mathcal{M}_{22}^{2}$ ). At tree level, $\mathcal{M}_{12}^{2}$ is small for small $m_{A}$ and/or large $\tan \beta$, but it cannot vanish. However, radiative corrections to $\mathcal{M}_{12}^{2}=-\left(m_{A}^{2}+m_{Z}^{2}\right) s_{\beta} c_{\beta}+\delta \mathcal{M}_{12}^{2}$ can be of the same order as its tree level value for small values of $m_{A}$ and large $\tan \beta$. Hence, it is possible for the oneloop contribution to approximately cancel the tree-level result (with two-loop corrections to $\mathcal{M}_{12}^{2}$ small compared to the corresponding one-loop result). For moderate or large values of $\tan \beta$, the vanishing of $\mathcal{M}_{12}^{2}$ [see eq. (42)] leads to the approximate numerical relation [152]:

$$
\left[\frac{m_{A}^{2}}{m_{Z}^{2}}+1\right] \simeq \frac{\mu x_{t} \tan \beta}{100 M_{S}}\left(2 a_{t} x_{t}-11\right)\left[1-\frac{15}{16 \pi^{2}} \ln \left(\frac{M_{S}^{2}}{m_{t}^{2}}\right)\right] .
$$

where $h_{t}, \alpha_{s}$ and the weak gauge couplings have been replaced by their approximate numerical values at the the electroweak scale. For low values of $m_{A}$ or large values of the squark mixing parameters, a cancellation can easily take place. 
If $\mathcal{M}_{12}^{2} \simeq 0$ and $\tan \beta$ is large (values of $\tan \beta \gtrsim 5$ are sufficient), the resulting pat teru of Higgs: couplings is easy to understand. In this limit, $\mathcal{M}_{11}^{2} \simeq m_{A}^{2}$ and $\mathcal{M}_{22}^{2} \simeq m_{h}^{\max }$, as noted at the end of Section 3.2.1. Two cases must be treated separately depending on the value of $m_{A}$. First if $m_{A}<m_{h}^{\text {rrrax }}$. then $\sin \alpha \simeq-1, \cos \alpha \simeq 0$ and $\sin \beta \simeq-\cos (\beta-\alpha) \simeq 1$. In this case, the lighter CP-even Higgs bosun $h$ is roughly aligned along the $\Phi_{d}^{0}$ direction and the heavier CP-even Higgs boson $H$ is roughly aligned along the $\Phi_{u}^{0}$ direction [see eq. (8)]. In particular, the coupling of $H$ to $b \vec{b}$ and $\tau^{+} \tau^{-}$is significantly diminished (since down-type fermions couple to $\Phi_{d}^{0}$ ), while the $H V \mathrm{~V}$ couplings [eq. (20)] are approxinately equal to those of the Standard Model [since $\cos ^{2}(\beta-\alpha) \simeq 1$ ]. Consequently, the branching ratios of $H$ into gg. $\gamma \gamma, c \bar{c}$, and $W^{+} W^{-}$can be greatly enhanced over Standard Nodel expectations [1;2.160,169.170]. Second, if $m_{4} \gg m_{h}^{\max }$ then $\sin \alpha \sim 0$ and $\sin \beta \sim \cos \alpha \simeq \sin (\beta-r \gamma) \simeq 1$ and the previnus considerations for $H$ apply now to $h$.

Although it is difficult to have an exact cancellation of the off-diagonal element $\mathcal{M}_{12}^{2}$. in many regions of the MSSM parameter space, a significant suppression of $\mathcal{M}_{12}^{2}$ may be present. Generically. the leading radiative corrections to $\mathcal{M}_{12}^{2}$ depend strongly on the sign of the product $\mu_{1} Y_{t}\left(A_{t} \simeq I_{t}\right.$ for large $\tan \beta$ and moderate $\mu)$ and on the value of $\left|A_{t}\right|$. For the same value of $X_{1}$. a change in the sign of $\mu$ can lead to observable variations in the branching ratio for the Higgs boson decay into boutom quarks. If $a_{1}^{2} \lesssim 11 / 2$, then the absolute value of $\mathcal{M}_{12}^{2}$ tends to be suppressed [enhanced] for values of $\mu t_{f} \therefore 0$ $\left[\mu A_{t}>0\right.$ ], which implies a sinilar suppression [emhancement] for the coupling of luolions quarks and T-leptons to the SM-like Higgs boson. For larger values of $\left|a_{t}\right|$, the suppression [enhancement] ocrurs for the opposite sign of $\mu A_{f}$

\subsubsection{The decoupling limit revisited}

Radiative corrections can also significantly affect the onset of the decoupling limit. Rocall that at. (ret: level [see eq. (18)], $|\cos (\beta-\alpha)| \ll 1$ for $m_{A} \gg m_{Z}$, in which case the couplings of $h$ are nearly identical to those of the SM Higgs boson. Including the effects of $\delta \mathcal{M}^{2}$, we use eq. (48) to obtain

$$
\begin{aligned}
\cos (\beta-\alpha) & =\frac{\left(\mathcal{M}_{11}^{2}-\mathcal{M}_{22}^{2}\right) \sin 2 \beta-2 \mathcal{M}_{12}^{2} \cos 2 \beta}{2\left(m_{H}^{2}-m_{h}^{2}\right) \sin (\beta-\alpha)} \\
& =\frac{m_{Z}^{2} \sin 4 \beta+\left(\delta \mathcal{M}_{11}^{2}-\delta \mathcal{M}_{22}^{2}\right) \sin 2 \beta-2 \delta \mathcal{M}_{12}^{2} \cos 2 \beta}{2\left(m_{H}^{2} \cdot m_{h}^{2}\right) \sin (\beta \quad \alpha)}
\end{aligned}
$$

Since $\delta \mathcal{M}_{i j}^{2} \sim \mathcal{O}\left(m_{Z}^{2}\right)$, and $m_{H}^{2}-m_{h}^{2}=m_{A}^{2}+\mathcal{O}\left(m_{Z}^{2}\right)$, one finds

$$
\cos (\beta-\alpha)=c\left[\frac{m_{Z}^{2} \sin 4 \beta}{2 m_{A}^{2}}+\mathcal{O}\left(\frac{m_{Z}^{1}}{m_{A}^{1}}\right)\right] .
$$

in the limit of $m_{A} \gg m_{Z}$, where

$$
c \equiv 1+\frac{\delta \mathcal{M}_{11}^{2}-\delta \mathcal{M}_{22}^{2}}{2 m_{Z}^{2} \cos 2 \beta}-\frac{\delta \mathcal{M}_{12}^{2}}{m_{Z}^{2} \sin 2 \beta} .
$$


Eq. (51) exhibits the expected decoupling behavior for $m_{A} \gg m_{Z}$. However, eq. (50) illustrates another way in which $\cos (\beta-\alpha)=0$ can be achieved - simply choose the MSSM parameters (that govern the Higgs mass radiative corrections) such that the numerator of eq. (50) vanishes. That is,

$$
2 m_{Z}^{2} \sin 2 \beta=2 \delta \mathcal{M}_{12}^{2}-\tan 2 \beta\left(\delta \mathcal{M}_{11}^{2}-\delta \mathcal{M}_{22}^{2}\right)
$$

Note that eq. (53) is independent of the value of $m_{A}$. For a typical choice of MSSM parameters, eq. (53) yields a solution at large $\tan \beta$. That is, by approximating $\tan 2 \beta \simeq-\sin 2 \beta \simeq-2 / \tan \beta$, one can determine the value of $\beta$ at which the decoupling occurs:

$$
\tan \beta \simeq \frac{2 m_{Z}^{2}-\delta \mathcal{M}_{11}^{2}+\delta \mathcal{M}_{22}^{2}}{\delta \mathcal{M}_{12}^{2}}
$$

The explicit expressions for $\delta \mathcal{M}_{i j}^{2}$ quoted in eq. (42) confirm that the assumption of $\tan \beta \gg 1$ used to derive this result is a consistent approximation because $\delta \mathcal{M}_{12}^{2}$ is typically small. We conclude that for the value of $\tan \beta$ specified in eq. (54), $\cos (\beta-\alpha)=0$ independently of the value of $m_{A}$. We shall refer to this phenomenon as $m_{A}$-independent decoupling. From eq. (42), it follows that explicit solutions to eq. (53) depend on ratios of MSSM parameters and are thus insensitive to the overall supersymmetric mass scale, modulo a mild logarithmic dependence on $M_{S} / m_{t}$.

\subsubsection{Corrections to tree-level Higgs-fermion Yukawa couplings}

We have seen in Section 3.3.1 that Higgs couplings are modified at one loop due to the renormalization of the CP-even Higgs mixing angle $\alpha$. Additional contributions from the one-loop vertex corrections to tree-level Higgs couplings must also be considered [171-174]. These corrections are typically small and therefore do not alter significantly the pattern of Higgs couplings. However, at large $\tan \beta$, the corrections to Higgs-fermion Yukawa couplings can be enhanced, and thus require a careful analysis.

In the supersymmetric limit, bottom quarks only couple to $\Phi_{d}^{0}$ and top quarks only couple to $\Phi_{u}^{0}$. However, supersymmetry is broken and a small coupling of the bottom quark [top quark] to $\Phi_{u}^{0}\left[\Phi_{d}^{0}\right]$ will be generated from the one-loop Yukawa vertex corrections. These results can be summarized by an effective Lagrangian that describes the coupling of the Higgs bosons to the third generation quarks: ${ }^{19}$

$$
-\mathcal{L}_{\mathrm{eff}}=\epsilon_{i j}\left[\left(h_{b}+\delta h_{b}\right) \bar{b}_{R} \boldsymbol{\Phi}_{\mathbf{d}}^{i} Q_{L}^{j}+\left(h_{t}+\delta h_{t}\right) \bar{t}_{R} Q_{L}^{i} \boldsymbol{\Phi}_{\mathbf{u}}^{j}\right]+\Delta h_{t} \bar{t}_{R} Q_{L}^{k} \boldsymbol{\Phi}_{\mathbf{d}}^{k *}+\Delta h_{b} \bar{b}_{R} Q_{L}^{k} \Phi_{\mathbf{u}}^{k *}+\text { h.c. }
$$

\footnotetext{
${ }^{19}$ Due to weak isospin breaking, one should allow for different radiatively induced couplings to charged and neutral Higgs bosons. For example, one should write $\Delta h_{b} \bar{b}_{R} b_{L} \Phi_{u}^{0 *}+\Delta \bar{h}_{b} \bar{b}_{R} t_{L} \Phi_{u}^{-}$in place of $\Delta h_{b} \bar{b}_{R} Q_{L}^{k} \boldsymbol{\Phi}_{u}^{k *}$, etc. To the extent that weak isospin breaking effects are small in the loop diagrams that generate $\Delta h_{b}$ and $\Delta \bar{h}_{b}$, it follows that $\Delta h_{b} \approx \Delta \bar{h}_{b}$ (and similarly for the other radiatively generated coefficients), and we may use eq. (55) as written.
} 
implying a modification of the tree-level relations between $h_{t}, h_{b}$ and $m_{t}, m_{b}$ as follows $[175,176,172,114$ :

$$
\begin{aligned}
& m_{b}=\frac{h_{b} v}{\sqrt{2}} \cos \beta\left(1+\frac{\delta h_{b}}{h_{b}}+\frac{\Delta h_{b} \tan \beta}{h_{b}}\right) \equiv \frac{h_{b} \theta}{\sqrt{2}} \cos \beta\left(1+\Delta_{t}\right) . \\
& m_{t}=\frac{h_{t} v}{\sqrt{2}} \sin \beta\left(1+\frac{\delta h_{t}}{h_{t}}+\frac{\Delta h_{t} \cot \beta}{h_{t}}\right) \equiv \frac{h_{t} v}{\sqrt{2}} \sin \beta\left(1+\Delta_{t}\right) .
\end{aligned}
$$

The dominant contributions to $\Delta_{b}$ are $\tan \beta$-enhanced, with $\Delta_{b} \simeq\left(\Delta h_{b} / h_{b}\right)$ tan $\beta ;$ for tan $\beta \gg 1, \delta h_{b} / h_{i}$ provides a small correction to $\Delta_{b}$. In the same limit, $\Delta_{t} \simeq \delta h_{t} / h_{t}$, with the additional contribution of

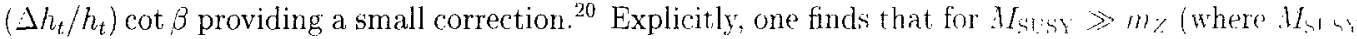
represents a typical supersymmetric mass that appears in the loops) and for tan $\rightarrow \gg 1 \mid 175,176.111$.

$$
\begin{aligned}
& \Delta_{b} \simeq\left[\frac{2 \alpha_{s}}{3 \pi} \mu M_{\tilde{g}} I\left(M_{\tilde{b}_{1}}^{2}, M_{\tilde{b}_{2}}^{2}, M_{\tilde{g}}^{2}\right)+\frac{h_{t}^{2}}{16 \pi^{2}} \mu A_{t} I\left(M_{\tilde{f}_{1}}^{2}, M_{\tilde{t}_{2}}^{2}, \mu^{2}\right)\right] \text { tan } \beta . \\
& \Delta_{t} \simeq-\frac{2 \alpha_{s}}{3 \pi} A_{t} M_{\tilde{g}} I\left(M_{\tilde{t}_{1}}^{2}, M_{\tilde{t}_{2}}^{2}, M_{\tilde{g}}^{2}\right)-\frac{h_{b}^{2}}{16 \pi^{2}} \mu^{2} I\left(M_{\tilde{b}_{1}}^{2}, M_{\tilde{t}_{2}}^{2}, \mu^{2}\right) .
\end{aligned}
$$

where $\alpha_{s} \equiv g_{3}^{2} / 4 \pi, M_{\tilde{g}}$ is the gluino mass, $M_{\tilde{b}_{1,2}}$ are the bottom squark masses. and smaller electroweak corrections have been ignored. The loop integral $I\left(a^{2} \cdot b^{2}, c^{2}\right)$ is given by

$$
I(a, b, c)=\frac{a^{2} b^{2} \ln \left(a^{2} / b^{2}\right)+b^{2} c^{2} \ln \left(b^{2} / c^{2}\right)+c^{2} a^{2} \ln \left(c^{2} / a^{2}\right)}{\left(a^{2}-b^{2}\right)\left(b^{2}-c^{2}\right)\left(a^{2}-c^{2}\right)} .
$$

and is of order $1 / \max \left(a^{2}, b^{2}, c^{2}\right)$ when at least one of its arguments is large compared to miz. Nut that the Higgs coupling proportional to $\Delta h_{b}$ is a manifestation of the broken supersymmetry in the low energy theory; hence, $\Delta_{b}$ does not decouple in the limit of large values of the supersymmetry breaking masses. Indeed, if all supersymmetry breaking mass parameters (and $\mu$ ) are scaled by a common factur. the correction $\Delta_{b}$ remains constant.

Similarly to the case of the bottom quark, the relation between $m_{\tau}$ and the Higgs-tau-lepton Yukawa coupling $h_{\tau}$ is modified:

$$
m_{\tau}=\frac{h_{\tau} \cdot d}{\sqrt{2}}\left(1+\Delta_{\tau}\right)
$$

The correction $\Delta_{T}$ contains a contribution from a tau slepton-neutralinu loop (depending on the iwo tau-slepton masses $M_{\tilde{\tau}_{1}}$ and $M_{\tilde{\tau}_{2}}$ and the mass parameter of the $\widetilde{B}$ component of the neutralino, $M_{1}$ )

\footnotetext{
${ }^{20}$ Because the one-loop corrections $\delta h_{b}, \Delta h_{b}, \delta h_{t}$ and $\Delta h_{t}$ depend only on Yukawa and gauge couplings and tlif supersymmetric particle masses, they contain no hidden $\tan \beta$ enhancements [177].
} 
and a tau sneutrino-chargino loop (depending on the tau sneutrino mass $M_{\bar{\nu}_{\tau}}$, the mass parameter of the $\widetilde{W}^{ \pm}$component of the chargino, $M_{2}$, and $\mu$ ). It is given by $[176,144]$ :

$$
\Delta_{\tau}=\left[\frac{\alpha_{1}}{4 \pi} M_{1} \mu I\left(M_{\tilde{\tau}_{1}}, M_{\bar{\tau}_{2}}, M_{1}\right)-\frac{\alpha_{2}}{4 \pi} M_{2} \mu I\left(M_{\bar{\nu}_{+}}, M_{2}, \mu\right)\right] \tan \beta
$$

where $\alpha_{2} \equiv g^{2} / 4 \pi$ and $\alpha_{1} \equiv g^{\prime 2} / 4 \pi$ are the electroweak gauge couplings. Since corrections to $h_{\tau}$ are proportional to $\alpha_{1}$ and $\alpha_{2}$, they are expected to be smaller than the corrections to $h_{b}$.

From eq. (55) we can obtain the couplings of the physical Higgs bosons to third generation fermions. The resulting interaction Lagrangian is of the form:

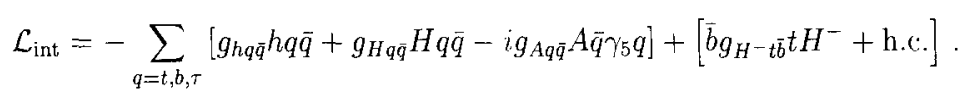

Using eqs. (56) and (57), one obtains:

$$
\begin{aligned}
g_{h b \bar{b}} & =-\frac{m_{b}}{v} \frac{\sin \alpha}{\cos \beta}\left[1+\frac{1}{1+\Delta_{b}}\left(\frac{\delta h_{b}}{h_{b}}-\Delta_{b}\right)(1+\cot \alpha \cot \beta)\right], \\
g_{H b \bar{b}} & =\frac{m_{b}}{v} \frac{\cos \alpha}{\cos \beta}\left[1+\frac{1}{1+\Delta_{b}}\left(\frac{\delta h_{b}}{h_{b}}-\Delta_{b}\right)(1-\tan \alpha \cot \beta)\right], \\
g_{A b \bar{b}} & =\frac{m_{b}}{v} \tan \beta\left[1+\frac{1}{\left(1+\Delta_{b}\right) \sin ^{2} \beta}\left(\frac{\delta h_{b}}{h_{b}}-\Delta_{b}\right)\right], \\
g_{h t \bar{t}} & =\frac{m_{t}}{v} \frac{\cos \alpha}{\sin \beta}\left[1-\frac{1}{1+\Delta_{t}} \frac{\Delta h_{t}}{h_{t}}(\cot \beta+\tan \alpha)\right] \\
g_{H t \bar{t}} & =\frac{m_{t}}{v} \frac{\sin \alpha}{\sin \beta}\left[1-\frac{1}{1+\Delta_{t}} \frac{\Delta h_{t}}{h_{t}}(\cot \beta-\cot \alpha)\right] \\
g_{A t \bar{t}} & =\frac{m_{t}}{v} \cot \beta\left[1-\frac{1}{1+\Delta_{t}} \frac{\Delta h_{t}}{h_{t}}(\cot \beta+\tan \beta)\right]
\end{aligned}
$$

and the $\tau$ couplings are obtained from the above equations by replacing $m_{b}, \Delta_{b}$ and $\delta h_{b}$ with $m_{\tau}$, $\Delta_{\text {, and }} \delta h_{\imath}$, respectively. In addition, one must employ the renormalized value of $\alpha$ in the above formulae to incorporate the radiative corrections discussed in section 3.3.1. In writing out the Higgstop quark couplings above, we found it convenient to express the results in terms of $\Delta_{t}$ and $\Delta h_{t} / h_{t}$, since $\Delta_{t} \simeq \delta h_{t} / h_{t}$ and the corresponding contribution of $\Delta h_{t} / h_{t}$ is $\tan \beta$ suppressed [eq. (57)]. Alternatively, eqs. (64)-(69) can be rewritten in a more symmetrical form by using eqs. (56) and (57) to eliminate $\Delta_{b}$ and $\Delta_{t}$ from the numerators of the corresponding expressions. 
At large $\tan \beta$, terms involving $\Delta_{b} \alpha \tan \beta$ [eq. (58)] provide the dominant corrections to the neutral Higgs couplings to $b \bar{b}$. The corrections proportional to $\delta h_{b} / h_{b}$ [see eqs. (55) and (56) and the discussion that follows] are never $\tan \beta$-enhanced and are therefore numerically unimportiant. The sign of $\perp_{\text {, is }}$ governed by the sign of $M_{\dot{g}} \mu$, since the bottom-squark gluino loop gives the dominant contribution tu, cq. (58). Thus, in a convention where $M_{\tilde{n}}>0$, the radiatively corrected coupling $9.16 \bar{t}$ is suppresued (enhanced) with respect to its tree level value for $\mu>0(\mu<0)$. In contrast, the radiative corrections to $g_{h b \bar{b}}$ and $g_{H b \bar{b}}$ have a more complicated dependence on the supersymmetric parameters due to the: dependence on the CP-even mixing angle $\alpha$. Since $\alpha$ and $\Delta_{b}$ are governed by different combinations of the supersymmetry breaking parameters, it is difficult to exhibit in a simple way the behavior uf the' radiatively corrected couplings of the CP-ever Higgs bosons to the bottom quarks as a function of the MSSM parameters.

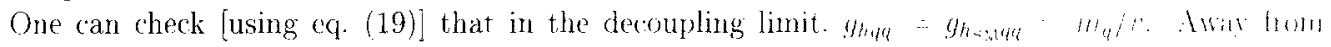
the decoupling limit, the Higgs couplings to botton-type fermions ada deviate signifiontly from thir tren-level values due to enhanced radiative corrections at large tan $t^{2}\left[\right.$ wher $\left.\Delta_{t} \simeq \mathcal{O}(1)\right]$. In particular. because $\Delta_{b}$ ox tan $\beta$, the leading one-loop radiative correction to $g_{h b \bar{h}}$ is of $\left.\mathcal{O}\left(m_{z}^{2} \text { tar } 3 / m\right)^{2}\right)$, which formally decouples only when $m_{A}^{2} \gg m_{Z}^{2} \tan \beta$. This behavior is called delayed decoupling in ref. [17 1 . In addition, there are regions of MSSM parameter space in which there is a strong suppression of th" Higgs coupling to $b \vec{b}$ (or $\tau^{+} \tau^{-}$) as compared to its tree--level value. As a result, thene can bo signitional

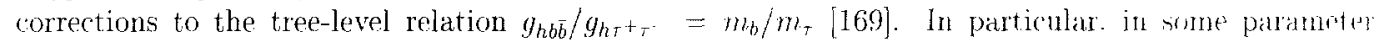
regimes, the $\tau^{+} \tau^{-}$decay mode can be the dominant $h$ decay chanmel a insult that would he fatel tw (ertain Higgs search strategies which assume that $h \rightarrow b \vec{b}$ is the dominant clecay und".

Assuming that weak isospin breaking effects in the loop corrections to the charged Higgs formion lukawa couplings are small (see footnote 19), then $y_{y}$ ij [defined in (9. (63)] is given by

$$
\begin{aligned}
& g_{H-t \bar{b}} \simeq \frac{\sqrt{2}}{r}\left\{m_{l} \cot \beta\left[1-\frac{1}{1+\Delta_{i}} \frac{\Delta h_{t}}{h_{l}}(\cot \beta+t a n)\right] P_{h}\right. \\
& \left.+m_{b} \tan \beta\left[1+\frac{1}{\left(1+\frac{1}{\Delta_{b}}\right) \sin ^{2} \beta}\left(\frac{\delta h_{b}}{h_{b}}-\Delta_{b}\right)\right] P_{i}\right\}
\end{aligned}
$$

with a similar form for $y_{H^{-} \nu_{\tau}+}+$ with the replacements noted below ect. $(69)$.

\subsubsection{Effects of explicit CP-violation}

In Section 3.2.3, we noted the possibility of mixing between the CP-even and CP-odd eigenstates due to CP-violating effects that enter via the one-loop radiative corrections. In this case, the neutral scalar mass eigenstates, denoted by $H_{i}(i=1,2,3)$, are determined by diagonalizing a $3 \times 3$ squared-mass matrix. Thus, one can no longer parameterize the various Higgs couplings in terms of the CP-even Higgs mixing angle $\alpha$. It is convenient to work in a convention where the two vacuum expectation 


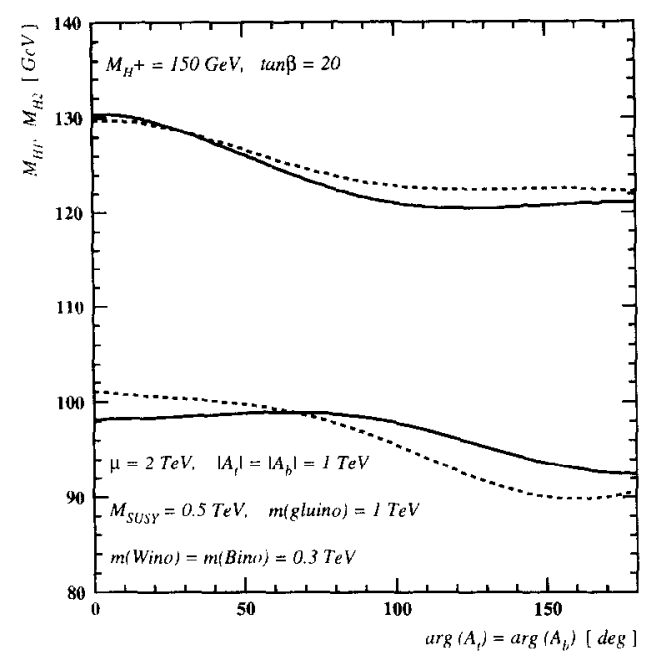

(a)

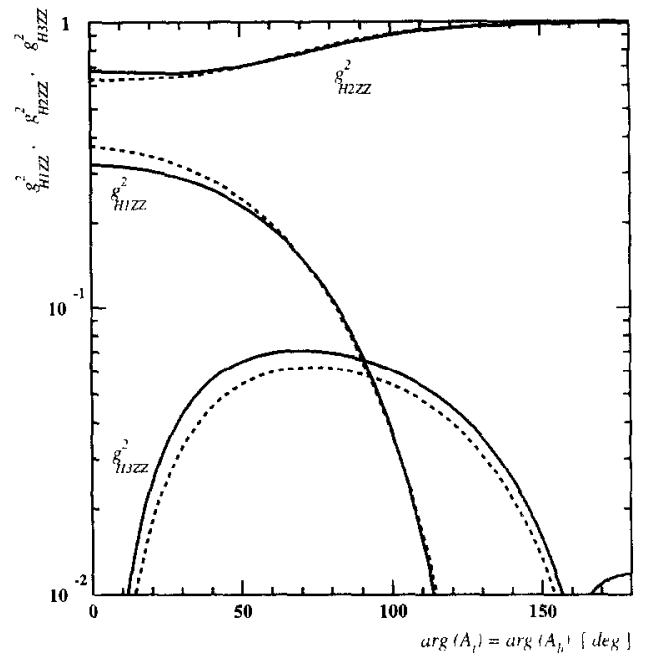

(b)

Figure 16: (a) Lightest and next-to-lightest neutral Higgs masses and (b) relative couplings (normalized to the SM) of the three neutral Higgs bosons to the $Z$ (or $W$ ) as a function of the phase of $A_{t}$ for the indicated choices of the MSSM parameters. Solid [dashed] lines are for $\arg \left(M_{\bar{g}}\right)=0^{\circ}\left[90^{\circ}\right.$. Taken from ref. [153].

values are real and positive (by absorbing any potential phases into the definition of the Higgs field) so that $\tan \beta=v_{u} / v_{d}$ as before. Then, eqs. (7) and (8) are replaced by

$$
H_{i}=\left(\sqrt{2} \operatorname{Re} \Phi_{d}^{0}-v_{d}\right) O_{1 i}+\left(\sqrt{2} \operatorname{Re} \Phi_{u}^{0}-v_{u}\right) O_{2 i}+\sqrt{2}\left(\operatorname{Im} \Phi_{d}^{0} \sin \beta+\operatorname{Im} \Phi_{u}^{0} \cos \beta\right) O_{3 i},
$$

where $O$ is a $3 \times 3$ real orthogonal matrix.

In the CP-violating case, vector boson pairs $V V(V=W$ or $Z)$ couple to all three neutral Higgs mass eigenstates, $H_{i}$, with [166]

$$
g_{H_{i} V V}=O_{1 i} \cos \beta+O_{2 i} \sin \beta .
$$

Fig. 16 shows the dependence of the Higgs masses and the $H_{i} Z Z$ squared-couplings on the phase of $A_{t}$ for a particular choice of MSSM parameters [as indicated in fig. 16(a)]. Clearly, these couplings can depend sensitively on the phases of the complex supersymmetry-breaking parameters that generate the mixing of the CP-even and CP-odd scalar eigenstates through one-loop radiative effects.

The couplings of $V$ to a pair of neutral Higgs bosons are given by $g_{H_{i} H_{j} Z}\left(p_{H_{i}}-p_{H_{j}}\right)$. wher the numenta $p_{H_{i}}$ and $p_{H_{3}}$ point into the vertex, $g_{H_{i} H_{j} Z}$ is antisymmetric under the interchange of $H_{i}$ and 
$H_{j}$, and $[166]$

$$
g_{H_{i} H_{j} Z}=\frac{g}{2 \cos \theta_{W}}\left[\left(O_{3 i} O_{1_{j}}-O_{1 i} O_{1 j}\right) \sin \beta-\left(O_{3 i} O_{2 j}-O_{2 i}()_{3 j}\right) \cos \beta\right] .
$$

Lising the orthogonality of $O$ (and $\operatorname{det} O=1$ ), it is easy to derive the relation [166]:"

$$
g_{H_{i} H_{1} Z}=\frac{m_{Z}^{2}}{2 m_{l}^{2}} \epsilon_{i j k} g_{H_{k} V^{\prime}} .
$$

The sum rules of eqs. (22)-(24) are then easily extended:

$$
\sum_{i} g_{H_{i} V V}^{2}=g_{V}^{2} m_{\tau}^{2}
$$

which when combined with eq. (74) yields

$$
\begin{gathered}
\sum_{i, j} g_{H_{i} H_{j} Z}^{2}=\frac{g^{2}}{2 \cos ^{2} \theta_{W}}, \\
g_{H_{i} V V} g_{H_{j} V V}+\frac{4 m_{V}^{4}}{m_{Z}^{2}} \sum_{k} g_{H_{i} H_{k} Z} g_{H_{j} H_{k} Z}=\frac{m_{V}^{4}}{m_{Z}^{2} \cos ^{2} \theta_{W}} \delta_{i j} .
\end{gathered}
$$

The couplings of a neutral Higgs boson to $H^{-} W^{+}$are given by $g_{H_{2} H^{-} W^{+}}\left(p_{H_{2}}-p_{H^{-}}\right)$, where the momenta $p_{H^{-}}$and $p_{H_{i}}$ point into the vertex, and [166]

$$
g_{H_{i} H^{-} W^{+}}=\left[g_{H_{i} H^{+} W^{-}}\right]^{*}=\frac{1}{2} g\left[O_{1 i} \sin \beta-O_{2 i} \cos \beta-i O_{3 i}\right] .
$$

Another consequence of the CP-violating effects in the scalar sector is that all neutral Higgs scalars can couple to both scalar and pseudoscalar fermion bilinear densities ( $\ddot{\psi} \psi$ and $\bar{\psi} \gamma_{\overline{5}} \psi$, respectively). The couplings of the mass eigenstate $H_{i}$ to fermions depend on the loop-corrected fermion Yukawa couplings, $h_{b, t}, \delta h_{b, t}, \Delta h_{b, t}$, and on $\tan \beta$ and the $O_{j i}$. It is convenient to adjust the phases of the fields so that the quantities $h_{b}+\delta h_{b}+\Delta h_{b} \tan \beta$ and $h_{t}+\delta h_{t}+\Delta h_{t} \cot \beta$ are both real and positive [i.e.. the physical fermion masses are still given by eqs. (56) and (57)]. The resulting expressions are a straightforward generalization of those presented above for the CP-conserving case [153]:

$$
\mathcal{L}_{H \bar{f} f}=-\sum_{i=1}^{3} H_{i}\left[\frac{m_{b}}{v} \bar{b}\left(g_{H_{i} h b}^{S}+i g_{H_{i} h h}^{P} \gamma_{5}\right) b+\frac{m_{t}}{v} \bar{t}\left(g_{H_{i} t t .}^{S}+i g_{H_{i} t t}^{P} \gamma_{5}\right) t\right]
$$

\footnotetext{
${ }^{21}$ One can easily check that eqs. (20) and (21) are recovered in the CP-conserving limit, where $\left(H_{1}, H_{2}, H_{3}\right)=(h, H . A$. $O_{22}=-O_{11}=\sin \alpha, O_{12}=O_{2,1}=\cos \alpha, O_{33}=1$, and all other elements $O_{j i}$ vanish.
} 
with

$$
\begin{aligned}
g_{H_{i} b b}^{S}= & \frac{1}{h_{b}+\delta h_{b}+\Delta h_{b} \tan \beta}\left\{\operatorname{Re}\left(h_{b}+\delta h_{b}\right) \frac{O_{1 i}}{\cos \beta}+\operatorname{Re}\left(\Delta h_{b}\right) \frac{O_{2 i}}{\cos \beta}\right. \\
& \left.-\left[\operatorname{Im}\left(h_{b}+\delta h_{b}\right) \tan \beta-\operatorname{Im}\left(\Delta h_{b}\right)\right] O_{3 i}\right\} \\
g_{H_{i} b b}^{I}= & \frac{1}{h_{b}+\delta h_{b}+\Delta h_{b} \tan \beta}\left\{\left[\operatorname{Re}\left(\Delta h_{b}\right)-\operatorname{Re}\left(h_{b}+\delta h_{b}\right) \tan \beta\right] O_{3 i}\right. \\
& \left.-\operatorname{Im}\left(h_{b}+\delta h_{b}\right) \frac{O_{1 i}}{\cos \beta}-\operatorname{Im}\left(\Delta h_{b}\right) \frac{O_{2 i}}{\cos \beta}\right\} \\
g_{H_{i} t t}^{S}- & \frac{1}{h_{t}+\delta h_{t}+\Delta h_{t} \cot \beta}\left\{\operatorname{Re}\left(h_{t}+\delta h_{t}\right) \frac{O_{2 i}}{\sin \beta}+\operatorname{Re}\left(\Delta h_{t}\right) \frac{O_{1 i}}{\sin \beta}\right. \\
& \left.-\left[\operatorname{Im}\left(h_{t}+\delta h_{t}\right) \cot \beta-\operatorname{Im}\left(\Delta h_{t}\right)\right] O_{3 i}\right\} \\
g_{H_{i} t t}^{P}= & \frac{1}{h_{t}+\delta h_{t}+\Delta h_{t} \cot \beta}\left\{\left[\operatorname{Re}\left(\Delta h_{t}\right)-\operatorname{Re}\left(h_{t}+\delta h_{t}\right) \cot \beta\right] O_{3 i}\right. \\
& \left.-\operatorname{Im}\left(h_{t}+\delta h_{t}\right) \frac{O_{2 i}}{\sin \beta}-\operatorname{Im}\left(\Delta h_{t}\right) \frac{O_{1 i}}{\sin \beta}\right\}
\end{aligned}
$$

where the Higgs scalar couplings are normalized with respect to the corresponding SM values.

For large values of the charged Higgs boson mass and for heavy supersymmetric particles, the expressions of the lightest neutral Higgs boson coupling to fermions reduce to those of the (CP-conserving) SM Higgs boson, as expected for the decoupling limit. In contrast, the two heavy neutral Higgs bosons are still admixtures of CP-even and CP-odd eigenstates; hence, CP-violating effects are still present in the heavy neutral Higgs sector. However, due to the high degeneracy in mass of the heavy scalar sector (especially in the decoupling limit), CP-violating effects may be difficult to observe without precision measurements of the heavy neutral Higgs properties.

The couplings of the charged Higgs bosons to fermions are of the form $\mathcal{L}_{\text {int }}=\bar{b} g_{H-t \bar{b}} t I I^{-}+$h.c. with

$$
g_{H-t \bar{b}} \simeq\left(\frac{\sqrt{2} m_{b}}{v} \tan \beta-\frac{\Delta h_{b}}{\cos \beta}\right) P_{L}+\left(\frac{\sqrt{2} m_{t}}{v} \cot \beta-\frac{\left(\Delta h_{t}\right)^{*}}{\sin \beta}\right) P_{R}
$$

One can check that for real $\Delta h_{b}$ and $\Delta h_{t}$, this result is equivalent to eq. (70) [with the same caveats noted in footnote 19]. An explicit computation of the CP-violating $H^{-} t \bar{b}$ vertex and its phenomenological implications can be found in ref. [178]. 


\subsection{MSSM Higgs Boson Decay Modes}

In the MSSM, we must consider the decay properties of three neutral Higgs bosous and one charged Higgs pair. ${ }^{22}$ In the region of parameter space where $m_{4} \gg m_{z}$ and the masses of supersymmetri. particles are large, the decoupling limit applies, and we find that the properties of $h$ are indistinguishabl: from the SM Higgs boson. If supersymmetric particles are light, then the decoupling limit dues not. strictly apply even in the limit of $m_{A} \gg m_{Z}$. In particular, the $h$ branching ratios are moditied. if tho decays of $h$ into supersymmetric particles are kinematically allowed. In addition, if light superpartners exist that can couple to photons and/or gluons, then the one-loop gg and $y$ decay lates would alson deviate from the corresponding Standard Model Higgs decav rates due to the extra contribution of the. light superpartners appearing in the loops. In both cases, the heavier Higgs states, $H, \perp$ and $H^{*}$, ane roughly mass degenerate, and their decay branching ratios depend crucially on tan $b$ as shown below.

For values of $m_{A} \sim \mathcal{O}\left(m_{Z}\right)$, all Higgs boson states lic below 200 GeV in mass. In this paranute regime, there is a significant area of the parameter space in which none of the neutral Higgs boson deray properties approximates that of the SM Higgs boson. For tan $3 \gg 1$, the resulting Higgs phenomenology shows marked differences from that of the SM Higgs boson [179]. In particular, radiative cormetious an significantly modify the $b \bar{b}$ and/or the $\tau^{+} \tau^{-}$decay rates with respect to those of the Sill ligge boson, as noted in Section 3.3.3. Additionally, the Higgs hosons can decay into nfw channels. rither containing lighter Higgs bosons or supersymmetric particles. In the following the deravs cf the uevtrat Higgs bosons $h, H$ and $A$ and the decays of charged Higgs bosons are discressed with particular emphasis on differences from Standard Model expectations. In the following discrission. we whitsit resultu five 1an $\beta=3$ and 30 to illustrate the difference betwen "low" and "high" tan 2 . The results shown helow include the effects of the dominant radiative corrections. which affert hoth the masses and the coupliuge of the Higgs sector as described in Sections 3.2 and 3.3 .

In order to display results for Higgs branching ratios, we must choose a be't of MSSM paranteters We fix $\tan \beta$ (for two representative choices) and vary $m_{\text {it }}$ from its LEP experimental lower bound of $90 \mathrm{GeV}$ up to $1 \mathrm{TeV}$. In addition, the gluino and MSSM squark mass parameters have been chosen to be

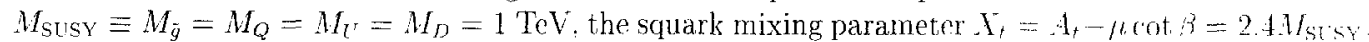
and the gaugino mass matrix parameters: $\mu=M_{2} \simeq 2 M_{1}=1$ ToV. This differs somewhat from 1 lo maximal mixing benchmark scenario of ref. [156]. Nevertheless, the value of $m_{h}$ is still close to maximal (lor fixed $m_{A}$ and $\tan b$ ), so we will continue to loosely refer to the above choice of MSSM parancoter as a maximal mixing scenario. Our motivation for choosing the gangino mass parameters large in in, avoid possible supersymmetric decay modes for the Higgs bosons for Higgs masses below 1 Tel. We shall briefly comment on possible supersymmetric decay modes at the end of this snctiont.

The branching ratios for $h$ and $H$ as a function of their masses are shown in fig. 17 As an a bates

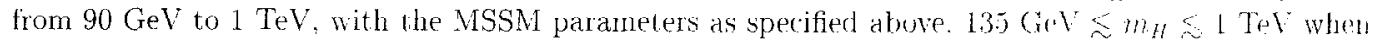

\footnotetext{
${ }^{22}$ Unless otherwise noted, wo shall neglect CP-viulating effects (e.4. by assuming that (P-violating efferts induced bx radiative corrections are small).
} 

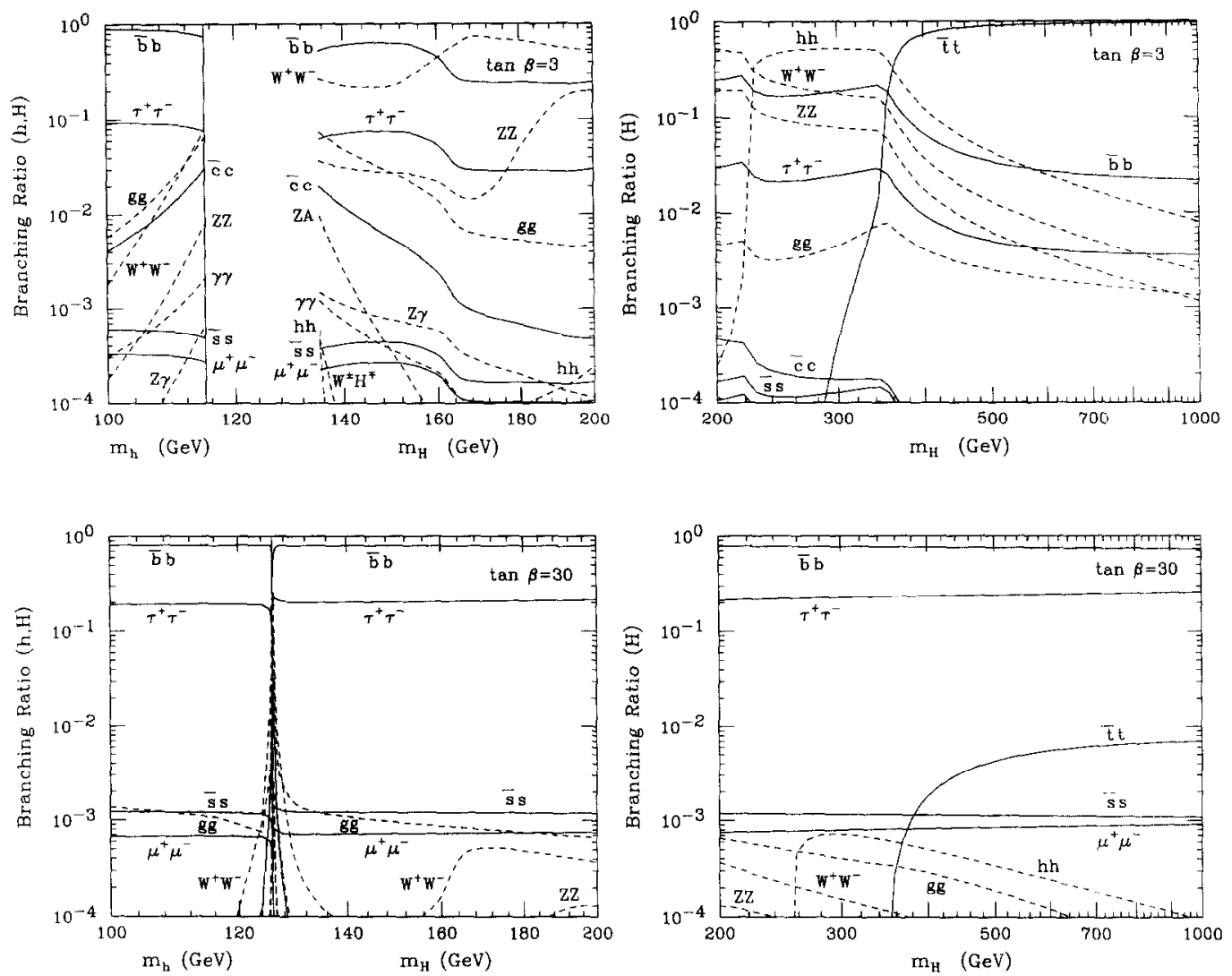

Figure 17: Branching ratios of the MSSM Higgs bosons $h$ and $H$, with $\tan \beta=3$ and 30 , respectively. Final states labeled above include the possibility of one off-shell final state particle below the corresponding two-particle decay threshold. The above plots were made under the assumption that the average top and bottom squark masses are $1 \mathrm{TeV}$ and top-squark nixing is maximal. In this case, $m_{h}^{\max } \simeq 115 \mathrm{GeV}(125.9 \mathrm{GeV})$ for $\tan \beta=3(30)$, corresponding to the limit of large $m_{A 4}$, is indicated by the vertical line in the two left-side plots. The range of $m_{H}$ shown corresponds to varying $m_{A}$ between $90 \mathrm{GeV}$ and $1 \mathrm{TeV}$, while $m_{h}>100 \mathrm{GeV}$ corresponds to $m_{A}>139 \mathrm{GeV}(104 \mathrm{GeV})$ for $\tan \beta=3(30)$. Other supersymmetric parameters have been chosen such that there are no supersymmetric particle decay modes in the Higgs mass ranges shown above. 

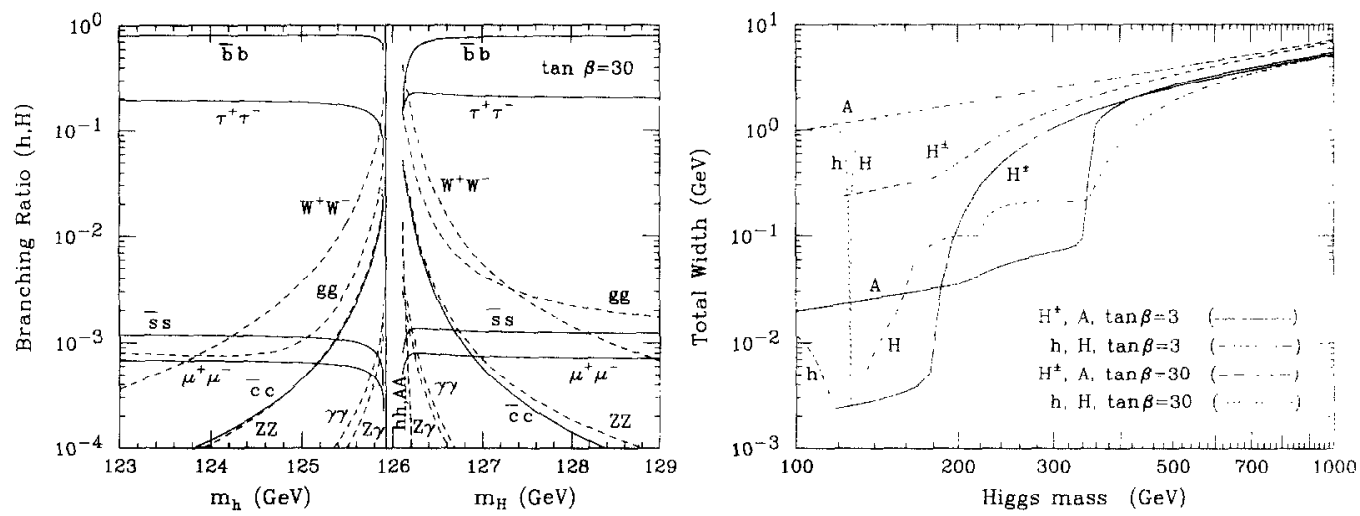

Figure 18: (a) Branching ratios of the MSSM Higgs bosons $h$ and $H$, with tan $\beta=30$. Here. we zoon in on the Higgs mass regime within $\pm 3 \mathrm{GeV}$ of $m_{h}^{\max }=125.9 \mathrm{GeV}$ of fig. 17 in order to get a clearer picture of the various deray modes. The range of $m_{H}$ shown corresponds to $90 \mathrm{GeV}<m_{A}<130 \mathrm{GeV}$, whereas the range of In shown corresponds to $128 \mathrm{GeV}^{2}<m_{A}<1 \mathrm{TeV}$. (b) Total widths of the MSSM Higgs boson as a function of the rorresponding Higgs mass for $\tan \beta=3$ and 30 , with the same parameter assumptions employed in fig. 17 .

$\tan \beta=3$ and $126.1 \mathrm{GeV} \lesssim m_{H} \lesssim 1 \mathrm{TeV}$ when $\tan \beta=30$. In contrast, most of the variation in $m_{1}$ occurs for values of $m_{h}$ a few $\mathrm{GeV}$ below $m_{h}^{\max }$. Thus, we also exhibit in fig. 18(a) the branching ration for $h$ and $H$ for $\tan \beta=30$ and Higgs mass values of $m_{h}^{\max } \pm 3 \mathrm{GeV}$. This reveals a detailed pattern of branching ratios that is not easily visible in fig. 17. The branching ratios for $A$ and $H^{+}$as a function of their masses are shown in fig. 19.

The total Higgs decay widths as a function of the corresponding Higgs mass are shown in fig. $18(b)$ for the two cases of $\tan \beta-3$ and 30 (and the other relevant MSSM parameters as described above). Note that for large values of the Higgs mass, the corresponding widths are considerably smaller than that of the SM Higgs boson. This is due to the suppressed $H V V$ couplings at large Higgs mass and to the absence of tree-level $A V V$ and $H^{+} W^{-} Z$ couplings. One can also check that in the decoupling limit $\left(m_{11} \gg m_{Z}\right)$, the total width of $h$ coincides with that of $h_{\mathrm{SM}}$. This is illustrated by replotting the $h$ (and $H$ ) widths on the same plot as the $h_{S M}$ width [see fig. 4(b)]. In particular, note that the dashed and dot-dashed $h$ contours in fig. 4 (b) approach the $h_{\mathrm{SM}}$ contour as $m_{h}$ reaches its maximal value. (which corresponds to the limit of large $m_{A}$ at fixed $\tan \beta$ ). It is interesting to note that in the opposite limit of small $m_{A}$ (especially at large $\tan \beta$ ) $\cos (\beta-\alpha) \rightarrow 1$ and it is $H$ that assumes many of the properties of $h_{\mathrm{SM}}$. However, there can still be deviations in the $H b \bar{b}$ coupling from the corresponding Standard Model value at large $\tan \beta$, as noted below eq. (35). This explains why the $H$ contours in fig. $4(b)$ 

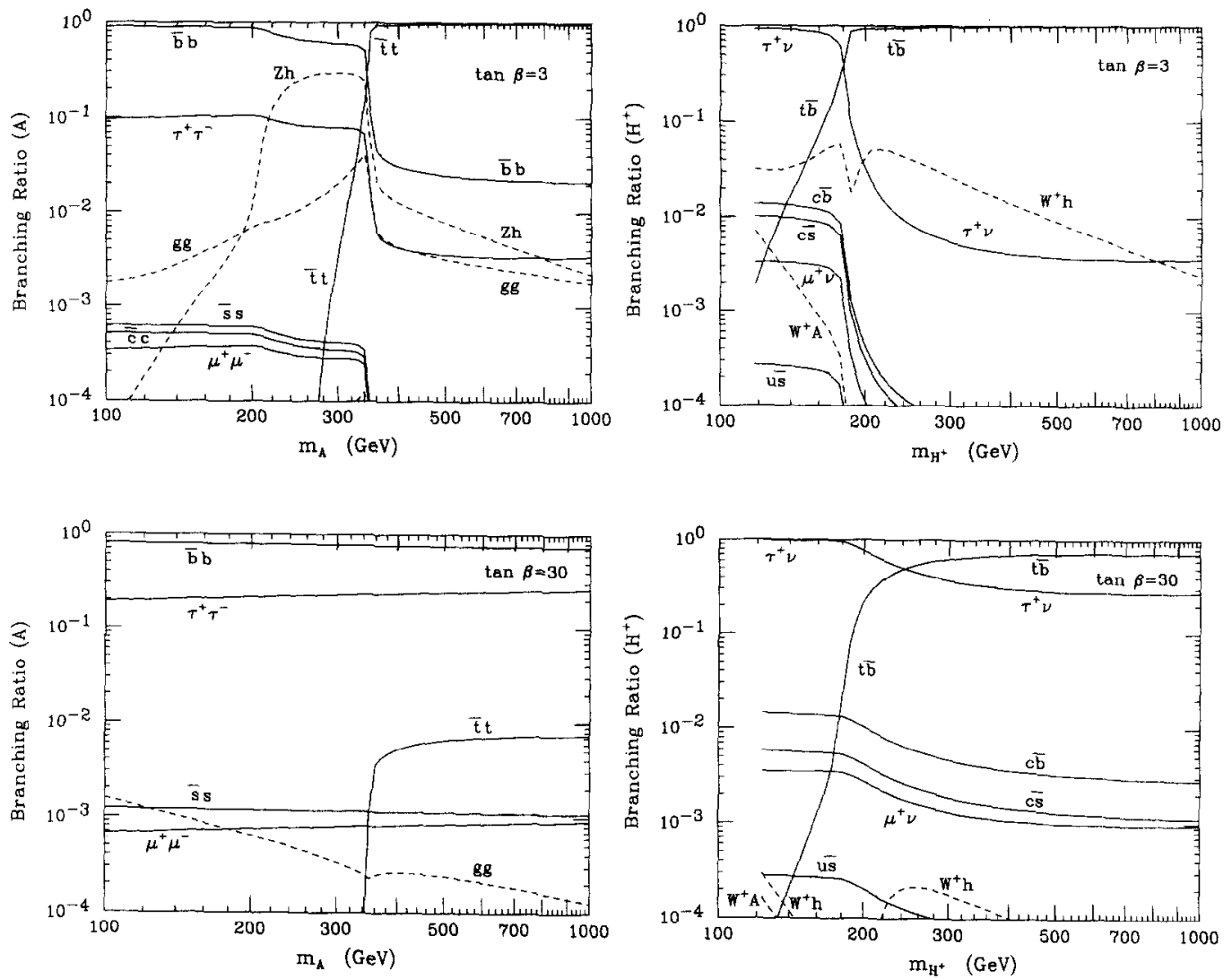

Figure 19: Branching ratios of the MSSM Higgs bosons $A$ and $H^{+}$, with $\tan \beta=3$ and 30 , respectively. Final states labeled above include the possibility of one off-shell final state particle below the corresponding two-particle decay threshold. The above plots were made under the assumption that the average top and bottom squark masses are 1 TeV and top-squark mixing is maximal. The range of $m_{H^{ \pm}}$shown corresponds to varying $m_{A}$ between $90 \mathrm{GeV}$ and $1 \mathrm{TeV}$. Other supersymmetric parameters have been chosen such that there are no supersymmetric particle decay modes in the IIiggs mass langes shown above. 
do not quite coincide with the result of the $h_{\mathrm{SM}}$ contour as $m_{H}$ approaches its lower limit (with the cliscrepancy between the $H$ and $h_{S M}$ contours more pronounced at large tan $\beta$ ).

The branching ratios and widths in figs. 17 -19 have been computed using a modified version ul the HDECAY program [180] that incorporates the leading radiative corrections to the Higgs couplings cliscussed in Section 3.3.2. The decay modes $h, I H, A \rightarrow b \vec{b}, \tau^{+} \tau$ dominate the nentral Higgs decay modes when $\tan \beta$ is large for all values of the Higgs masses. For small tan $\beta$, these modes are significant for neutral Higgs masses below $2 m_{t}$ (although there are other competing modes in this mass range). whereas the $t \bar{t}$ decay mode dominates above the tz decay threshold. In contrast to the SM Higgs boson. the vector boson decay modes of $H$ are strongly suppressed at large $m_{1 / f}$ dne to the suppressed $H 1 T$ rouplings in the decoupling limit. For the charged Higgs busim. $H^{+}+\tau^{\prime}{ }^{\prime}$ dominates below th threshold, while $H^{+} \rightarrow t \bar{b}$ dominates for large values of $m_{H^{t}}$, Nute that final states labeled in figs. 17 and 19 include the possibility of one off-shell final state particle belew the corresponding two-partick decay threshold [181]. For example, for $m_{h}=<m_{h}+m_{h}$, the $/ t_{\text {f }}$ contour shown in fig. 19 actually corresponds to an off-shell $/$ cuark that decays to $b W^{*}$. That is. in this mass region the to contur

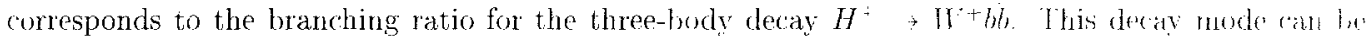
especially significant at moderate values of $\tan \beta$ due to the large Higgs-top s rakawa coupling

As in the Standard Model case, the partial decay widths of the neutral Higgs bonsons inte bo ancl a. are reduced by about 50 $75 \%$ when QCD corrections are included ( $\quad$ \% . by amploying running quark masses in the decay width formulae). whereas the QCD (corrections are less significant for Higgs decays into $t \bar{f}$ [29]. The effects of the QCD radiative corrections on the charged Higgs hrandhing ratine $182_{-}^{\circ}$ arr significant in the region of tan $\beta$ where the es and $\tau^{+} \nu_{y}$ decay modes are conpertitive on for large values

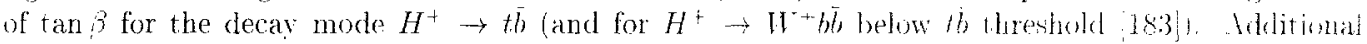
supersymmetric radiative corrections discussed in Section 3.3 an also significantly affect the Higgs boson partial widths. Some of these corrections can be absorbed into the offertive nixing angle of $1 / \mathrm{i}_{3}$ as shown in Section 3.3.1. As a consequence of this universal rorrections. the conpling of $h$ to $b i t$ ind

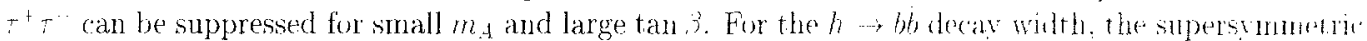
corrections $\left[\begin{array}{lll}152,171 & 174\end{array}\right]$ proportional to the strong coupling cunstant ${ }^{*}$, and the Higgs-top) quarli Yukawa coupling $h_{4}$ can be significant for large values of $\mu$ and tan i. As shewn in Section 33.3 .3 . thit: effect can be interpreted as a correction to the trew-level relation hetwetn $m_{i}$ and $h$ h.

In addition to the decay modes of the neutral Higgs bosens into fermion and gange lown fitian states, there exist new Higgs decay channels that involve scalars of the extended Higgs sectur and supersymmetric final states. The unambiguous observation of these modes (as well as any decay morle of a charged scalar) would clearly constitute direct evidence of new physis beyond the Standard Modnl. Higgs decays into charginos. neutralinos and third-generation squarks and sleptons can becone impor-

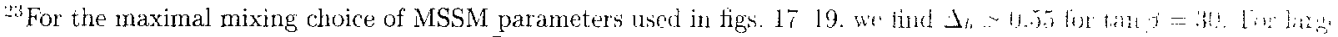

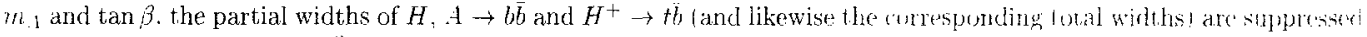
by a factor of about $\left(1+\Delta_{n}\right)^{2}$ with respect to the corresponding tree-level resultis
} 
tant, once they are kinematically allowed [184]. One interesting possibility is a significant branching ratio for the decay of a neutral Higgs boson to the invisible mode $\tilde{\chi}_{1}^{0} \tilde{\chi}_{1}^{0}$ (where $\tilde{\chi}_{1}^{0}$ is the lightest supersymmetric particle). In such a scenario, the discovery of this neutral Higgs boson would be difficult at a hadron collider [185]. In contrast, at lepton colliders, methods exist for detecting an invisibly decaying Higgs boson by observing a peak in the missing mass recoiling against the produced $Z$.

\subsection{MSSM Higgs Boson Production at Hadron Colliders}

\subsubsection{Cross-sections at hadron colliders}

The production mechanisms for the SM Higgs boson at hadron colliders can also be relevant for the production of the MSSM neutral Higgs bosons. However, we must take into account the possibility of enhanced or suppressed couplings (with respect to those of the Standard Model). For example, the $H V T$ couplings are very suppressed in the decoupling limit, and tree-level $A V T$ couplings are conipletely absent. On the other hand, at large $\tan \beta$, typically two of the three neutral Higgs couplings to bottom-type quarks are enhanced. These effects can significantly modify the neutral Higgs production cross-sections. New production mechanisms must be considered for charged Higgs production

As in the case of Iliggs branching ratios, the predicted cross-sections are sensitive to the MSSM Higgs parameters. Again, we consider two representative values of $\tan \beta$ : a low value of $\tan \beta=3$ and a high value of $\tan \beta=30$. We then vary $m_{A}$, evaluate the other Higgs masses, and compute each Higgs cross-section as a function of the corresponding Higgs mass. The Higgs masses and cross-sections depend on other MSSM parameters through radiative corrections. As in Section 3.4, we work in a maximal squark mixing scenario in which the value of $m_{h}$ for a fixed choice of $\tan \beta$ and $m_{A}$ is maximal. In addition, because the squark masses are assumed to be heavy (of order $1 \mathrm{TeV}$ ), potential supersymmetric contributions to the one-loop Higgs-gluon-gluon vertex (due to squark loops) are suppressed. Cross-sections for neutral MSSM Higgs production at the Tevatron and the LHC are shown in figs. 20) and 21 respectively. The dominant Higgs production mechanism over much of the MSSM parameter space is gluon-gluon fusion, which is mediated by heavy top and bottom quark triangle loops and the ('orresponding supersymmetric partners [186-188]. The gluon-gluon fusion results shown in figs. 20 and 21 include NLO QCD corrections [189]. ${ }^{24}$

The cross-sections for the production of the neutral CP-even Higgs bosons $(\phi=h$ or $H$ ) via gauge boson fusion $V^{*} V^{* *} \rightarrow \phi(V=W$ or $Z)[45]$ and via the process $q \bar{q} \rightarrow V^{*} \rightarrow V \phi$ [42], including firstorder QCD corrections, ${ }^{25}$ are also exhibited in figs. 20 and 21 . Recall that the CP-even scalar $\phi$ has S.M-like couplings to the vector bosons in two cases: (i) in the decoupling regime for the lightest Higgs boson, where $\phi=h$ and (ii) for large $\tan \beta$ and low $m_{A}$, where $\phi=H$. In either case, the SM-like

\footnotetext{
${ }^{24}$ A recent computation of the NNLO QCD corrections to $A$ production via gluon fusion exhibits a $20-30 \%$ increase orer the corresponding NLO cross-section [190].

${ }^{25}$ The supersymmetric-QCD corrections due to the exchange of virtual squarks and gluinos are known to he small [188].
} 

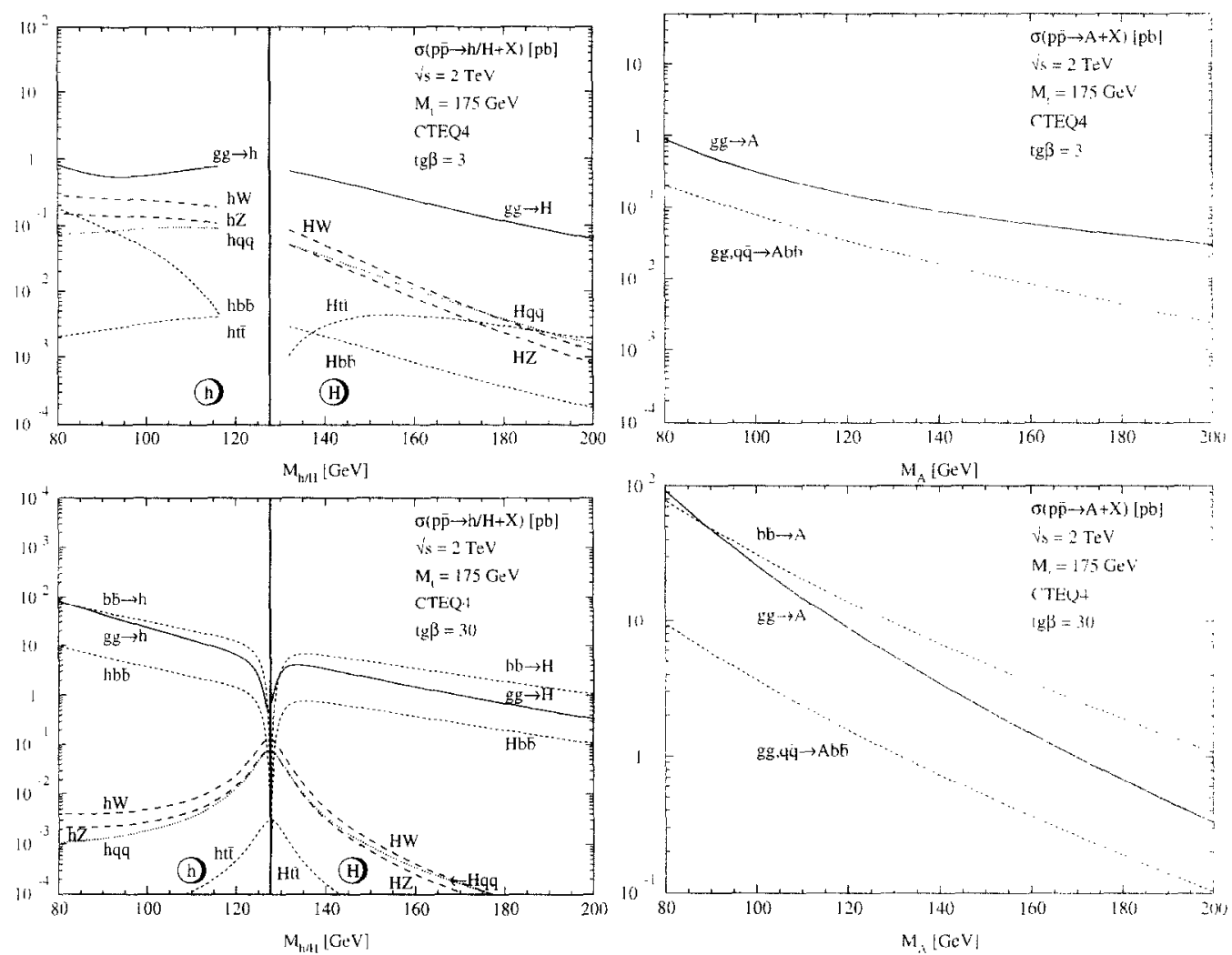

Figure 20: Neutral MSSM Higgs production cross-sections at the Tevatron $[\sqrt{s}=2$ TeV $]$ for gluon fusion $y g \rightarrow \rightarrow$ vector-boson fusion $q q \rightarrow q q V^{*} V^{*} \rightarrow q q h, q q H$, vector-boson bremsstrablung $q \bar{q} \rightarrow V^{*} \rightarrow h L / H V$ and the associated production $g g, q \bar{q} \rightarrow \phi b \bar{b} / \phi t \vec{t}$ including all known QCD corrections, where $\phi=h, H$ or $A[32,34]$. As in fig. $j$. in the vector boson fusion process, $q q$ refers to both $u d$ and $q \bar{q}$ scaltering. The four panels exhibiled abuve show (a) $h$. $H$ produclim for $\tan \beta=3$, (b) $A$ production for $\tan \beta=3$, (c) $h, H$ production for $\tan \beta=30$, (d) A produrtion fur $\tan \beta=30$ 

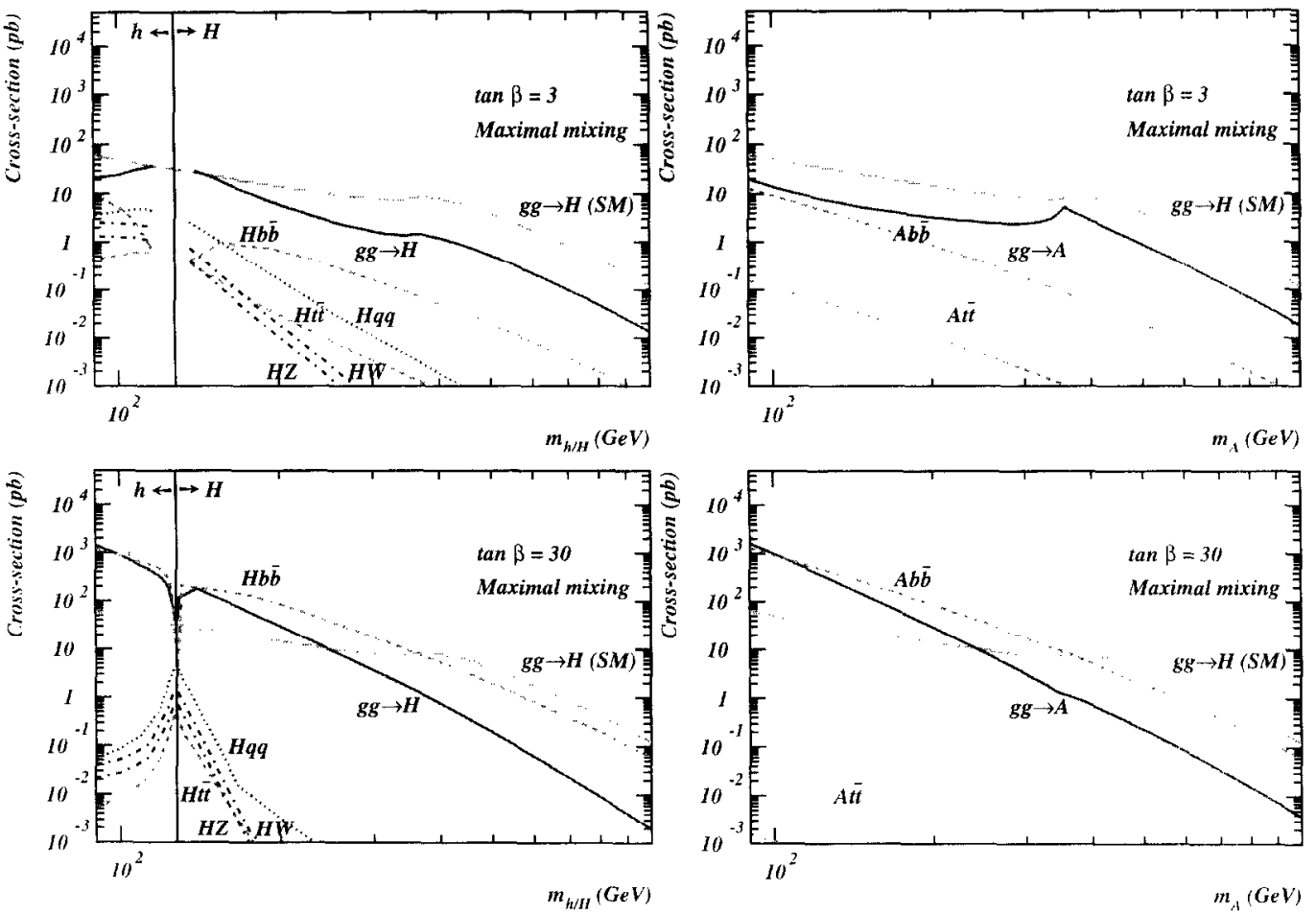

Figure 21: Neutral MSSM Higgs production cross-sections at the LHC $[\sqrt{s}=14 \mathrm{TeV}]$ fur gluon fusion $g g \rightarrow \phi$, vectorboson fusion $q q \rightarrow q q V^{*} V^{*} \rightarrow q q h, q q H$, vector-boson bremsstrahlung $q \bar{q} \rightarrow V^{*} \rightarrow h V / H V$ and the associated production $g g, q \vec{q} \rightarrow \phi b \bar{b} / \phi t \bar{t}$ including all known QCD corrections, where $\phi=h, H$ or $A[34,44]$. The four panels exhibited above show the cross-section in $\mathrm{pb}$ vs. the Higgs mass, ranging from $90 \mathrm{GeV}$ to $1 \mathrm{TeV}$, for (a) $h, H$ production for tarl $\beta=3$, (b) $A$ production for $\tan \beta=3$, (c) $h, H$ production for $\tan \beta=30$, (d) $A$ production for $\tan \beta=30$. For comparison, the cross-section for gluon-gluon fusion to a SM Higgs boson is also shown. 
Higgs scalar, $\phi$, has a mass less than or about equal to $130 \mathrm{Gel}$. and the curresponding cruss-seramm for $I^{* *} I^{*} \rightarrow \phi$ and $q \bar{q} \rightarrow I^{*} \rightarrow V \phi$ are phenomenologically relevant. The other (non-SM-like) CP-fven scalar has suppressed couplings to $\mathrm{I} \mathrm{T}$, and the corresponding cross-sections are generally too small to be observed.

Higgs boson radiation off bottom quarks becomes important for large tan $y$ in the MSSM. wher the? Higgs coupling to bottom-type fermions is enhanced. Thus, the theoretical predictions, including full NLO computations, are crucial for realistic simulations of the MSSM Higgs signals in these channels. ${ }^{21}$ Moreover, as discussed in Section 3.3.3, vertex corrections to the bbo coupling play a very importiant. role in enhancing or suppressing (depending on the MSSM parameters) these production cross-sertion at large $\tan \beta[152,160.170]$.

We now turn to charged Higgs production. If $m_{h}<m_{t}-m m^{ \pm}$, then $H^{\dagger}$ ran be produced in th: decay of the top quark via $\rightarrow b H^{-}$(and $\left.\bar{t} \rightarrow b H^{-}, 192\right]$. Tho $t \rightarrow b H^{+}$decay mode can be competitive

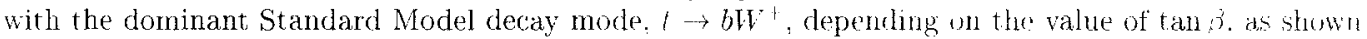
in fig. $22(\mathrm{a})$ for $m_{H^{t}}=120 \mathrm{GeV}$. This figure, taken from ref. [193] illustrates the effects of including

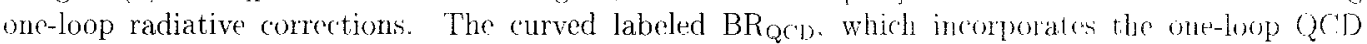
corrections (first computed in ref. [194]), is applicable to a more genclal (nom-supersymetric) Type-lI two-Higgs doublet model [based on the tref-level Higgs-fermion colnplings of opt. (28) (35)?. Voto that

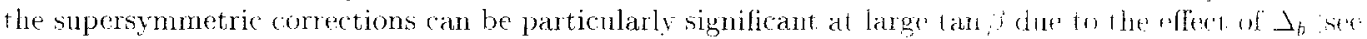

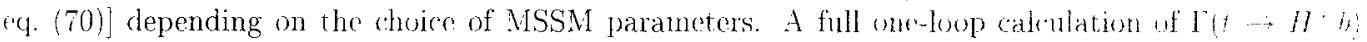
in the MSSM including all sources of large Yukawa couplings can be found in rets. $\mid 195]$ and $193 \mid$ a treatment including resummation of the leading QCD quantum offects and the dominant contribution from loop effects arising from supersymmetric particle exchange "an be found in ref. [] 96$]$

For $m_{H^{ \pm}}<m_{t} \cdot m_{b}$, the trotal cross-scetion for charged Higgs production fiu the narrow-widu approximation) is then given by

$$
\sigma\left(p \bar{p} \rightarrow H^{-c}+\mathrm{X}\right)=\left(1-\left[\mathrm{BR}\left(t \rightarrow b \mathrm{~V}^{-+}\right)^{2}\right) \sigma(\mu \eta) \rightarrow H+\ldots\right.
$$

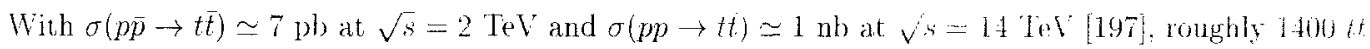
pairs per detector will be produced per year in Run 2a of the Tevatron (assuming a yearly luminusity of $2 \mathrm{fb}^{-1}$ ), while about $10^{7} 10^{8} t \bar{t}$ pairs will be produced at the LHC (assuming a yearly luminosity af $10100 \mathrm{fb}^{-1}$ ). Folding in the top quark branching ratio. it is a simple matter to compute the inclusive

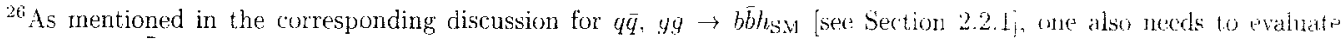

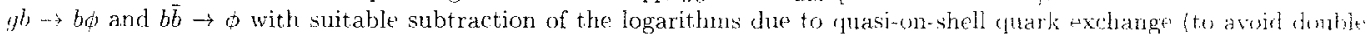

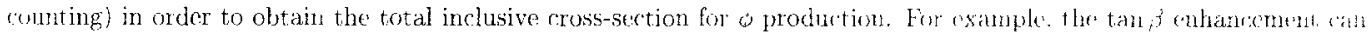
lead to copious s-channel production of Higgs bosons via $b$-quark fusion $[36,191]$.

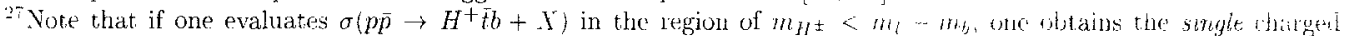
Higgs inclusive cross-section, $\sigma\left(p \bar{p} \rightarrow H^{+}+\mathrm{Y}\right)=\mathrm{BR}\left(t \rightarrow b H^{-}\right) \sigma(p \bar{p} \rightarrow t \bar{f}+\mathrm{X})$, wather than full charged Higgs inclusive' cruss-section of eq. (85). The latter is not quite a factor of two larger than the former since $F$ wan contain a a harged Higgs boson; one must subtract off $\left[\mathrm{BR}\left(t \rightarrow b H^{+}\right)\right]^{2} \sigma(p \bar{p} \rightarrow t \bar{t}+\mathrm{X})$ to avid dould $\mathrm{t}:-$ counting.
} 

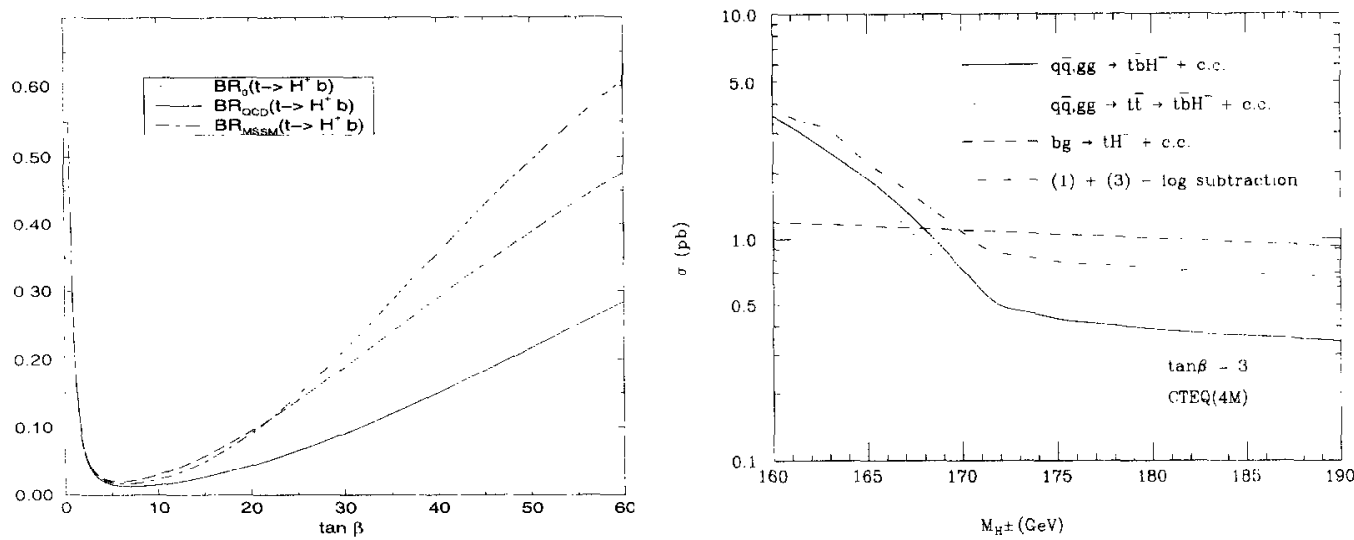

Figure 22: (a) Branching ratio for $t \rightarrow b H^{+}$in the MSSM as a function of $\tan \beta$ for $m_{H^{ \pm}}=120$ GeV. The three curves shown are the results of a computation that (i) is at tree-level; (ii) includes one-loop QCD corrections; and (iii) incorporates both one-loop QCD, electroweak and the effects of MSSM particle exchange (taken from ref. [193]); the parameters chosen in (iii) correspond to a rather light supersymmetric spectrum: $M_{\bar{g}}=300 \mathrm{GeV}, M_{\bar{t}}=100 \mathrm{GeV}$, $M_{\tilde{b}_{1}}=150 \mathrm{GeV}, A_{t}=A_{b}=300 \mathrm{GeV}, M_{2}=150 \mathrm{GeV}$, and $M_{\bar{u}}=M_{\bar{\nu}}=200 \mathrm{GeV}$. Curves (i) and (ii) are also applicable to a Model-II two-Higgs doublet model without supersymmetry. (b) The charged Higgs production cross-section at the LHC near the threshold for $t \rightarrow b H^{+}$for $\tan \beta=3$ (taken from ref. [199]).

(harged Higgs cross-section. For values of $m_{H^{ \pm}}$near $m_{t}$, the width effects are important. In addition, the full $2 \rightarrow 3$ processes $p \bar{p} \rightarrow H^{+} \bar{t} b+X$ and $p \bar{p} \rightarrow H^{-} t \bar{b}+X$ must be considered. In this case eq. (85) no longer provides an accurate estimate of the charged Higgs cross-section [198], as illustrated in fig. 22(b) (taken from ref. [199]). The results of fig. 22(a) imply that for $m_{H^{ \pm}}<m_{t}-m_{b}$, the discovery of the charged Higgs boson at the Tevatron and/or LHC (given sufficient luminosity) is possible if $\tan \beta \gg 1$ or $\tan \beta \lesssim 1$ (the latter is theoretically disfavored). The precise bound on $\tan \beta$ (as a function of $m_{H^{ \pm}}$) depends somewhat on the details of the other MSSM Higgs parameters.

If $m_{H^{ \pm}}>m_{t}-m_{b}$, then charged Higgs boson production occurs mainly through radiation off a third generation quark. Single charged Higgs associaled production proceeds via the $2 \rightarrow 3$ partonic processes $g g, q \bar{q} \rightarrow t \bar{b} H^{-}$(and the charge conjugate final state). As in the case of $b \bar{b} h_{\mathrm{SM}}$ production, large logarithms $\ln \left(m_{H^{ \pm}}^{2} / m_{b}^{2}\right)$ arise for $m_{H^{ \pm}} \gg m_{b}$ due to quasi-on-shell $t$-channel quark exchanges, which can be resummed by absorbing them into the $b$-quark parton densities. Thus, the proper procedure for computing the charged Higgs production cross-section is to add the cross-sections for $g b \rightarrow t H^{-}$ and $g g \rightarrow t \bar{b} I^{--}$and subtract out the large logarithms accordingly from the calculation of the $2 \rightarrow 3$ 

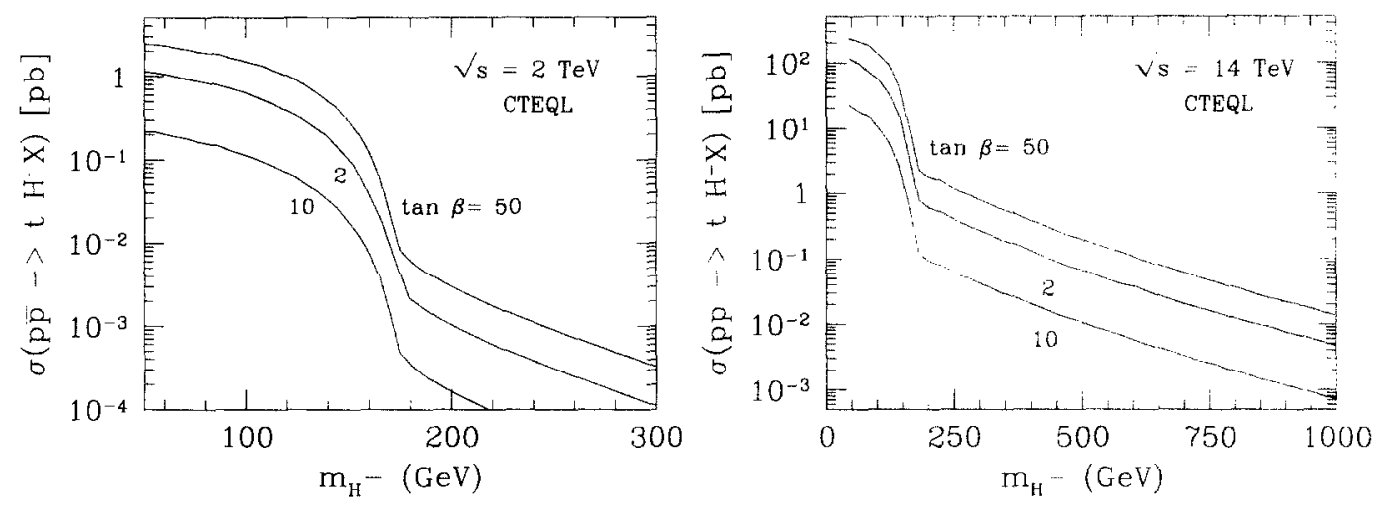

Figure 23: The leading-order production cruss-sections for charged Higgs production at (a) the levatron $(p \bar{p} \rightarrow t b H \rightarrow$ 1) and (b) the LHC $\left(p \bar{p} \rightarrow t \bar{b} H^{-}+X\right)$ are shown as a function of $m_{H^{ \pm}}$for three values of $\tan \beta=2.10$ and 5 (1). The: cross-sections are obtained by adding the contribution of the $2 \rightarrow 2$ processes, $y b \rightarrow \imath H^{*}$, to those of the $2 \rightarrow 3$ processes. $g g \rightarrow t \bar{b} H^{-}$and $q \bar{q} \rightarrow t \bar{b} H^{-}$(suitably subtracted to avoid double counting). Renormalization and factorization scales have been both set to $m_{t}+m_{H^{ \pm}}$. These results are taken from ref. [201].

process $[48,200]$. This procedure avoids double-counting of the large logarithms at $\mathcal{O}\left(a_{k}\right)$, and correct $1 \mathrm{y}$ resums the leading logs to all orders. In particular, the contribution to the total cross-section coming from the kinematical region of the gluon-initiated $2 \rightarrow 3$ process in which one of the two gluons splits into a pair of $b$-quarks (one of which is collinear with the initial proton or antiproton). is incorporated into the $b$-quark parton density. A cruder calculation would omit the contribution of the $2 \rightarrow 2$ process and simply include the results of the unsubtracted $2 \rightarrow 3$ process. The latter procedure would miss the resummed leading logs that are incorporated into the $b$-quark density. However, the numerical difference between the two procedures is significant only for $m_{H^{ \pm}} \gg m_{t}$.

The single inclusive charged Higgs cross-sections at the Tevatron and LHC are exhibited in fig. 2.3 as a function of the charged Higgs mass, for $\tan \beta=2,10$ and 50 . Note that the cross-sections shown include the region of charged Higgs mass below $m_{H^{ \pm}}=m_{t}-m_{b}$ corresponding to the case discussed above where the charged Higgs cross-section is dominated by $t \bar{t}$ production followed by $t \rightarrow b H^{-}$. These results are based on the calculations of ref. [201] and include the contributions of the $2 \rightarrow 2$ process and suitably subtracted $2 \rightarrow 3$ process as described above. Similar results have also been obtained in ref. [202]. The impact of the leading electroweak and MSSM radiative corrections has been studied in ref. [203]. In addition, the NLO QCD corrections to the $2 \rightarrow 2$ process $g b \rightarrow H^{+}+$have recently been 

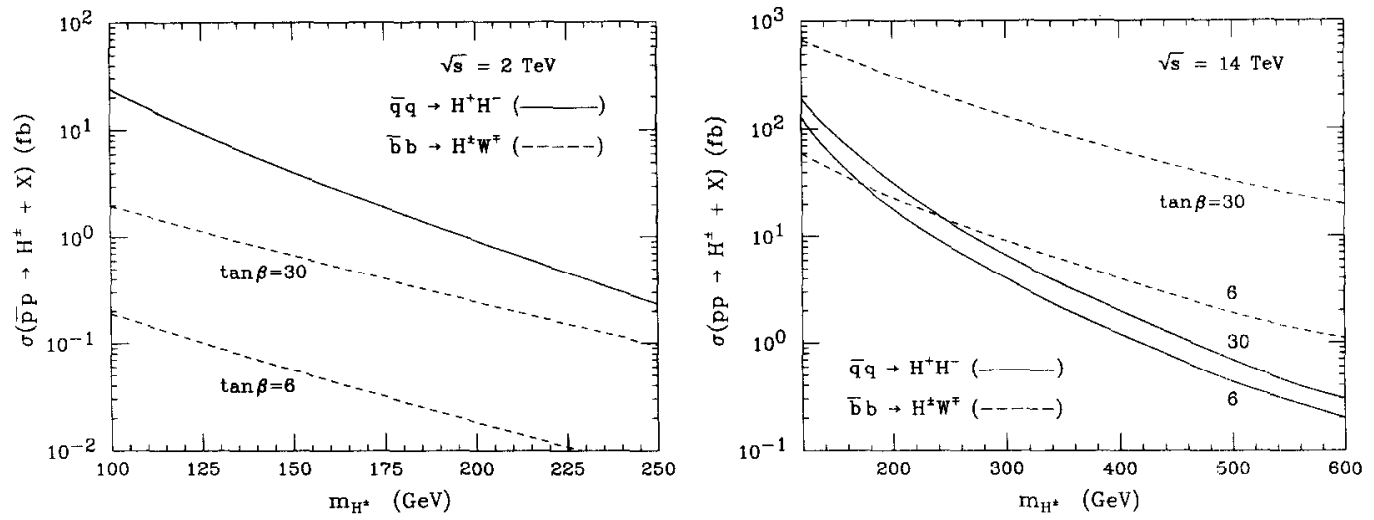

Figure 24: Total cross-section (in fb) for inclusive production of (i) $H^{+} H^{-}+X$ [207] (solid line) and (ii) $H^{ \pm} W^{\mp}+X[205]$ (dashed lines) as a function of $m_{H^{ \pm}}$for $\tan \beta=6$ and 30. Curves for (a) $p \bar{p} \rightarrow H^{+} H^{-}+X$ at the Tevatron and (b) $p p \rightarrow H^{+} H^{-}+X$ at the LHC are exhibited. Note that the dependence of process (i) on $\tan \beta$ is negligible at the Tevatron. while there is some $\tan \beta$ dependence at the LHC due to the enhancement of $b \bar{b} \rightarrow H^{+} H^{-}$at large tan $\beta$. The contribution of $b \bar{b}$ annihilation to process (ii) dominates over the $g g$ fusion scattering mechanism. $M_{t}=174.3 \mathrm{GeV}$ and a fixed $b$-quark pole mass of $M_{b}=4.7 \mathrm{GeV}$ are used to fix the Higgs-fermion Yukawa coupling. The leading-order CTEQ5I. parton distribution functions are used.

evaluated [204]. These corrections typically increase the tree-level cross-section by a factor of 1.3 to 1.6 , depending on the value of the charged Higgs mass and $\tan \beta$, with some additional dependence on the choice of renormalization and factorization scales.

Associated production of a charged Higgs boson and a $W^{ \pm}$can occur via $b \bar{b}$ annihilation and $g g$ fusion [205]. The contribution of $b \bar{b}$ annihilation to $\sigma\left(p \bar{p} \rightarrow H^{ \pm} W^{\mp}+X\right)\left[\sigma\left(p p \rightarrow H^{ \pm} W^{\mp}+X^{\prime}\right)\right]$ at the Tevatron [LHC] (both charge combinations are included) are shown as function of the charged Higgs mass for $\tan \beta=6$ and 30 in fig. 24. The loop-induced $g g$ fusion contribution is significantly suppressed relative to the tree-level $b \bar{b}$ annihilation if $\tan \beta \gtrsim 6$, independently of the value of $m_{H^{ \pm}}$[206].

Charged Higgs bosons can also be produced in pairs via Drell-Yan $q \bar{q}$ annihilation. The dominant contribution, which arises from $u \bar{u}$ and $d \bar{d}$ annihilation into a virtual photon or $Z$, is independent of $\tan \beta$. Some $\tan \beta$ dependence enters through $b \bar{b}$ annihilation via $t$-channel top-quark exchange, although this effect is more than one order of magnitude suppressed relative to the dominant contribution at the Tevatron. The $b \bar{b}$ annihilation is more significant at the LHC (at large $\tan \beta$ where the $H^{-} t \bar{b}$ coupling is enhanced). The tree-level results for $\sigma\left(p \bar{p} \rightarrow H^{+} H^{-}+X\right)$ at the Tevatron and $\sigma\left(p p \rightarrow H^{+} H^{-}+X\right)$ at the LHC are shown in fig. 24. These results are obtained [207] with the Higgs-fermion Yukawa 
coupling based on a fixed $b$-quark pole mass of $M_{b}=4.7 \mathrm{GeV}$. The contribution of the loop-indu'ed $y g \rightarrow H^{+} H^{-}$is typically less important than that of $q \bar{q}$ annihilation [208.209], and is not included in fig. 24. However, the $g y$ fusion contribution can become significant at large tan 6 . with further 'nhancements in some regions of MSSM parameter space in which the squarks (which appear in the loop) are light and strongly mixed [209]. Nevertheless, the inclusise $H^{-1} H^{-}$(ross-section lios beluw 1 ho (ross-section for single charged Higgs associated production (c.f. figs. 23 and 21 )

Finally, one can compute the cross-sections for double neutral Higgs production at hadron colliderts. These include the inclusive production of $h h . h H, H H . h A, H A$ and A.A. Cons-sections can be found in refs. [54 56] and [210,211] (QCD corrections to these (moss-sections aw avaluated in tef. [5ib). In

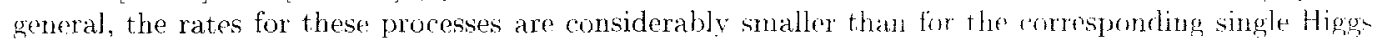
production rates. However, in certain regions of supersymmetric paraneter ipace sfuark loops ram anhance the cross-section for pair production of two CP-even Higgs bosons by as much ab two urderti of magnitude [210]. In some cases, observation of double Higgs production provides some information on three-Higgs couplings. For example, for low to morlerate values of tans. gluon fusion tw a virtual

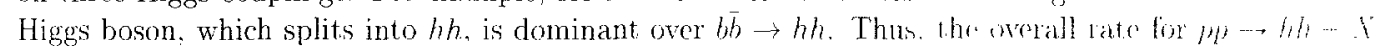
would provide a measure of the $h h h$ vertex.

Additional sources for Higgs boson production can arise from the decay of supersymmetric partirls. into final states containing one or more Higgs boson in the decay chain [212]. Therse procesisen deperd in detail on the details of the supersymmetric particle spectrum and their conplings. For example, the production of $h$ in supersymmetric particle decay followed by the clecay $h \rightarrow b \bar{b}$ (an yield a signal ahove background at LHC [213]. Processes of this type provide additional chamnels for possible Higgs discovery and precision study. and descrve further analysis.

\subsubsection{Benchmarks for Higgs searches}

In the search for the MSSM Higgs bosons, one must first search for the lightest Higgs scalar. which is expected (in almost all cases) to be the neutral CP-even scalar, $h$. In the decoupling region of the MSSM Higgs parameter space (where $m_{A} \gg m_{Z}$ ), the search techniques already outlined for $h_{i, i}$ are relevant for $h$, since the properties of $h$ approximately coincide with those of the SM Higgs boson. The $h$ discovery reach can be mapped out as a region of $m_{A^{-}}-\tan \beta$ parameter space, since these two parameters (along with the MSSM parameters that determine the size of the radiative corrections) fix the value of $m_{h}$. Next, it is critical to identify deviations of the properties of $h$ from those of $h_{\text {SM }}$. Positive evidence for such a deviation would signal the existence of additional scalar states of the non-minimal Higssertor. The difficulty of this step depends on how close the model is to the decoupling limit. After the discovery of $h$, the Higgs boson search will focus on the non-minimal Higgs states of the modrl.

for values of $m_{A} \sim m_{\%}$, all the Higgs bosons of the MSSM are of a similar order of magnitude. am the properties of $h$ will no longer resemble those of $h_{\text {SMY }}$. In principle, one can then discover multiple? scalar states in one experiment. Since the two CP-even scalars share the coupling to vector hosion pairs 
eq. (22)], one may identify the CP-even scalar whose squared-coupling to $V V$ is larger than $0.5 g_{h_{5 \mathrm{M}} V W}^{2}$. The Tevatron and LHC production cross-sections of this scalar (compared to that of $h_{\mathrm{SM}}$ ) are reduced by no more than $50 \%$ (by assumption), while the Higgs branching ratio into $b \bar{b}$ is similar to that of $h_{S M}$ over most of the MSSM parameter space. Thus, the Tevatron and LHC SM Higgs search results also apply here modulo minor modifications (which account for the somewhat suppressed production cross-section and the effects of supersymmetric corrections to the third generation Yukawa couplings).

The general MSSM parameter space involves many a priori unknown parameters. In practice, only a small subset of these parameters govern the properties of the Higgs sector. Nevertheless, a full scan of this reduced subset is still a formidable task. However, a detailed study of a few appropriately chosen points of the parameter space can help determine the ultimate MSSM Higgs discovery reach of the Tevatron and LHC. It is convenient to choose a set of benchmark MSSM parameters that govern the Higgs radiative corrections $[156,214]$. These include the supersymmetric Higgs mass parameter $\mu$, the third generation squark mixing parameters, $A_{t}$ and $A_{b}$, the gluino mass $M_{\bar{g}}$, the diagonal softsupersymmetry-breaking third generation squark squared-masses (which we take for simplicity to be degenerate and equal to $M_{\text {SUSY }}$ ), and the top quark mass (which is held fixed at $m_{t}=174.3 \mathrm{GeV}$ ). The maximal mixing benchmark scenario, is defined as the one in which the squark mixing parameters are such that they maximize the value of the lightest CP-even Higgs boson mass for fixed values of $m_{A}, \tan \beta$ and $M_{\text {SuSY }}$. Here, we choose $X_{t} \equiv A_{t}-\mu \cot \beta \simeq \sqrt{6}, A_{b}=A_{t}, M_{2}=-\mu=200 \mathrm{GeV}$ and $M_{\tilde{y}}=M_{\text {SusY }}=1 \mathrm{TeV}$ [corresponding to $\left.m_{h}^{\max }=129 \mathrm{GeV}\right]$.

The maximal mixing scenario poses a challenge for Higgs searches, since the predicted Higgs mass takes on its maximal value for a given set of MSSM parameters. However, different regions of the MSSM Higgs parameter space pose new challenges. For example, regions of parameter space exist in which the CP-even neutral Higgs boson with SM-like couplings l,o the $W, Z$ and $\ell$ has suppressed couplings to $b \bar{b}$. The benchmark scenario denoted by "suppressed $V \phi \rightarrow V b \bar{b}$ production" is an example of this behavior. In this case, we take $\mu=-A_{t}=1.5 \mathrm{TeV}, A_{b}=0, M_{2}=200 \mathrm{GeV}$ and $M_{\bar{g}}=M_{\text {SuSY }}=1 \mathrm{TeV}$ [corresponding to $m_{h}^{\max }=120 \mathrm{GeV}$ ]. The regions of strongly suppressed $\mathrm{BR}(\phi \rightarrow b \bar{b})$ correspond to a suppressed $H b \bar{b}$ coupling at lower $m_{A}$ and a suppressed $h b \bar{b}$ coupling at larger $m_{A}$. In particular, the suppression for large $\tan \beta$ extends to relatively large values of $m_{A} \sim 300 \mathrm{GeV}$, indicating a delay in the onset of the decoupling limit. Moreover, in the suppressed $V \phi \rightarrow V b \vec{b}$ benchmark scenario, all the Higgs couplings to $b \bar{b}$ are generally suppressed, since $0<\Delta_{b} \ll 1$ and $\sin 2 \alpha \simeq 0$. From the analytic formulae, it can be deduced that $\mu A_{t}<0$ and large values of $\left|A_{t}\right|,|\mu|$ and $\tan \beta$ are needed.

The coverage in the $m_{A}-\tan \beta$ plane by different Higgs production and decay channels can vary significantly, depending on the choice of MSSM parameters. In the last example in which the CP-cven Higgs boson with the larger coupling to the $W$ and $Z$ has a strongly suppressed coupling to bottom quarks, the Higgs searches at the Tevatron will become more problematical, while the LHC search for Higgs production followed by its decay into photons becomes more favorable [160]. At the same time the LHC Higgs discovery reach via vector bosons fusion to Higgs production followed by its decay into $\tau^{+} \tau^{-}$pairs can be significant [215]. 


\subsubsection{MSSM Higgs Boson searches at the Tevatron}

We first consider the Tevatron MSSM Higgs search. Specifically, we make use of the Tevatron $h$ sM search techniques, where $h_{\mathrm{SM}}$ is replaced by either $h$ or $H$. If $\tan \beta \gg 1$, a new search mode becomes viable, due to the possibility of enhanced couplings of the neutral Higgs boson stiates to $b \bar{b}[216,170]$. Thus, we also consider the possibility of the $b \bar{b} \phi \rightarrow b \bar{b} b \bar{b}$ signature, where $\phi=h, H$, and $/$ or $A$. If tan $\phi$ is large, two of the neutral Higgs boson states, $\phi=A$ and $h[H]$ are produced with enhanced rates if $m \_\leqslant m \%$ [ $\left.m_{A} \gg m_{Z}\right]$, as noted below eq. (35). We may combine the results for the various chanmels to provide summary plots of the MSSM Higgs discovery reach of the upgraded Tevatron collider. We considey here the results based on a generic MSSM analysis [62]; see ref. [217] for a similar allalysis in the context of a varicty of models of supersymmetry breaking. In the latter case, the results obrained will be somewhat more constraining than the generic analysis, since the supersymmetry-loreaking parameters that control the radiative corrections to Higgs masses and couplings are no longer arbitrary.
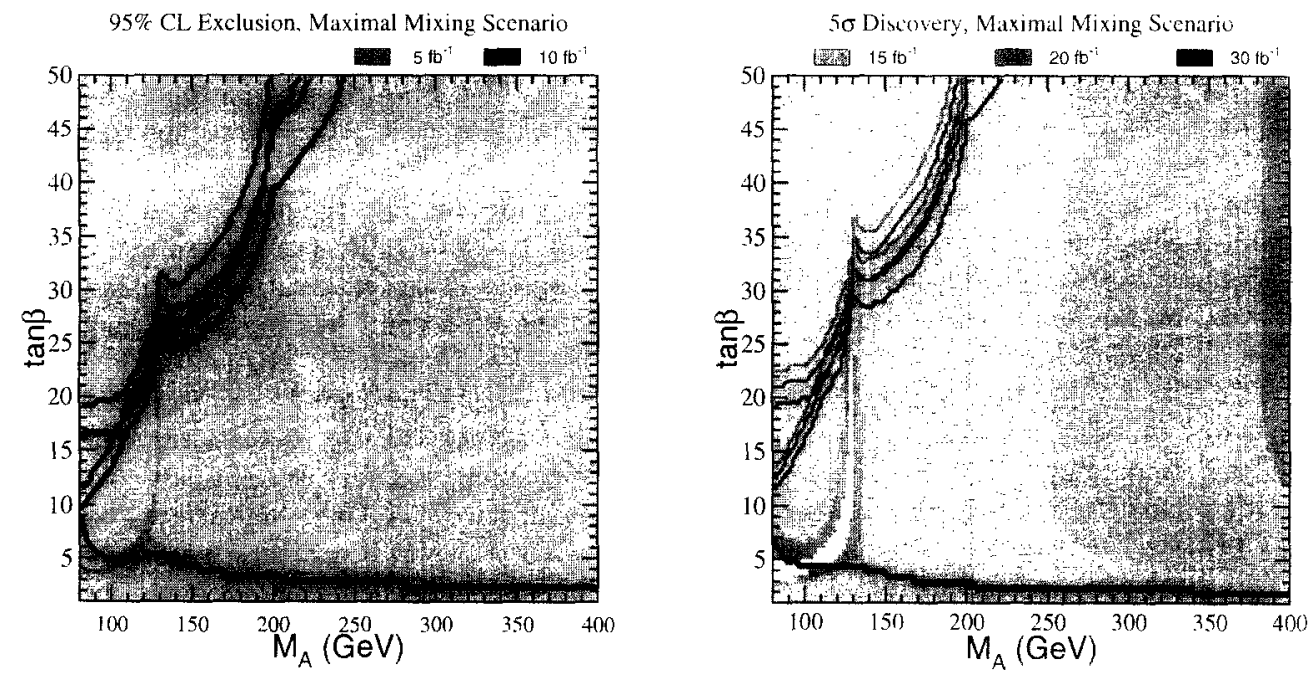

Figure 25: (a) 95\% CL exclusion region and (b) $5 \sigma$ discovery region in the ne a dn flant. for tho maximal nixing benchunark scenario (see Section 3.5.2) and two different search channels: $q \bar{q} \rightarrow I^{*} \omega[\phi=h . H], \dot{\phi} \rightarrow b \bar{b}$ (shaded regions) and $g g, q \bar{q} \rightarrow b \bar{b} \phi[\phi=h, H, A], \phi \rightarrow b \bar{b}$ (region in the upper left-hand corner bounded by the solid lines: the two sets of lines correspond to CDF and DO simulations). The region below the solid black line near the bottom of the plot is excluded by the absence of $e^{+} e^{-} \rightarrow Z \phi$ events at LEP. Taken from ref. [62]. 

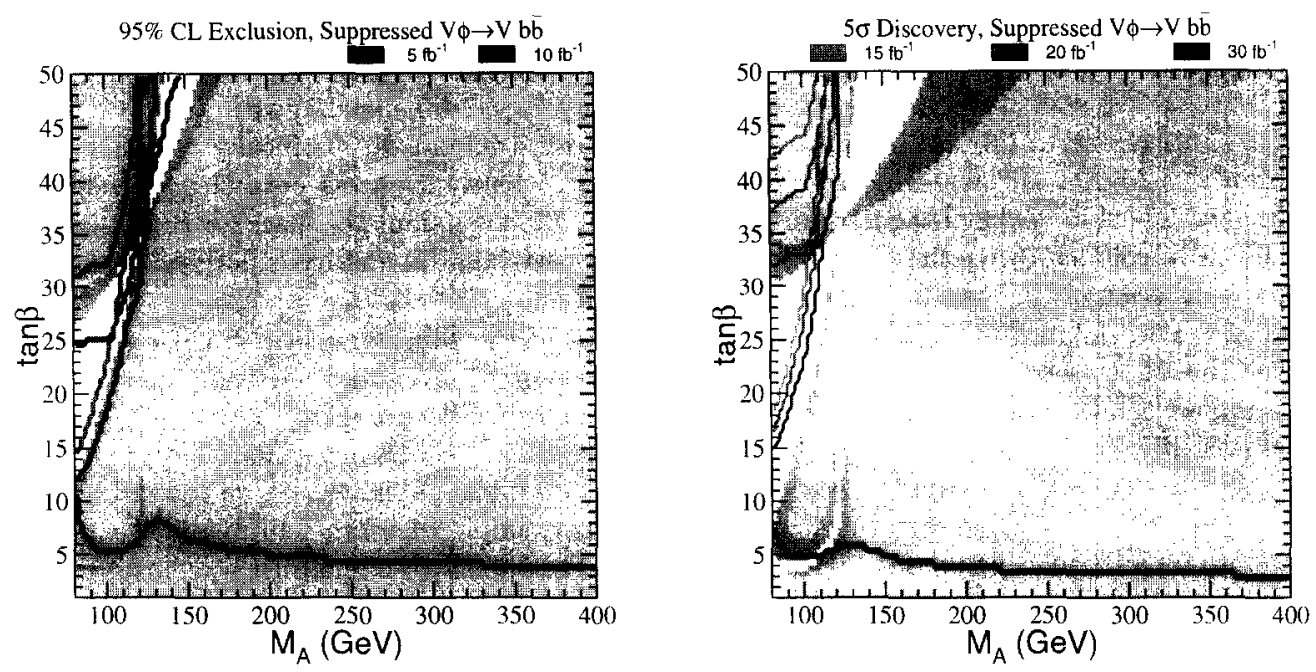

Figure 26: The same as fig. 25 but for the suppressed $V \phi \rightarrow V b \bar{b}$ production benchmark scenario of Section 3.5.2. Taken from ref. [62].

In figs. 25 and 26 , we show the regions of $95 \%$ CL Higgs exclusion and $5 \sigma$ Higgs discovery on the $m_{A} \cdot \tan \beta$ plane, for two representative MSSM parameter choices, via the search of neutral Higgs busons in the channels: $q q \rightarrow V \phi[\phi=h, H], \phi \rightarrow b \bar{b}$ (shaded regions) and $g g, q q \rightarrow b \bar{b} \phi[\phi=h, H, A], \phi \rightarrow b \bar{b}$ (region in the upper left-hand corner bounded by the solid lines), for different integrated luminosities as indicated by the color coding. The shaded regions presented in these figures reflect the results of the SHW simulation of $q \bar{q} \rightarrow V \phi$ improved by neutral network techniques [62]. The two sets of lines (for a given color) bounding the regions accessible by the $b \bar{b} \phi$ search correspond to the CDF and DØ simulations, respectively. The solid black line near the bottom of each plot indicates the lower limit of $\tan \beta$ (as a function of $m_{A}$ ) based on the absence of observed $e^{+} e^{-} \rightarrow Z \phi$ events at LEP [161]. Note the importance of the complementarity between the $q \bar{q} \rightarrow V \phi$ and $q \bar{q} \rightarrow b \bar{b} \phi$ channels for improving the coverage of the MSSM parameter space in the low $m_{A}$ region in fig. 26(a). The results of figs. 25(a) and $26\left(\right.$ a) demonstrate that $5 \mathrm{fb}^{-1}$ of integrated luminosity per experiment will be sufficient to cover nearly all of the MSSM Higgs parameter space at $95 \%$ CL in the benchmark scenarios specified above.

To assure discovery of a CP-even Higgs boson at the $5 \sigma$ level, the luminosity requirement becomes very important. Figs. 25(b) and 26(b) show that a total integrated luminosity of about $20 \mathrm{fb}^{-1}$ per 
experiment is necessary in order to assure a significant, although not exhaustive, coverage of the MSSM parameter space. In general, we observe that the complementarity between the two channels, $y \bar{q} \rightarrow 1$ and $q \bar{q} \rightarrow b b \phi$, is less effective in assuring discovery of a Higgs boson as compared with a $95 \%$ CL Higgs exclusion. This is due to the much higher requirement of total integrated luminosity combined with the existence of MSSM parameter regimes which can independently suppress both Higgs production channels. Fig. 26 exhibits one of the most difficult regions of MSSM parameter space for Higgs searches at the Tevatron collider. Nevertheless, even in this case, a very high luminosity experiment can corer i significant fraction of the available MSSM parameter space.

If explicit $\mathrm{CP}$ violation occurs through nonzero phases of the supersymmetry breaking paramelers, then the three neutral Higgs bosons are a combination of CP-even and CP-odd states and th: phenomenology can become much more complicated. In particular the couplings of the neutral Higgo busons to the $W$ and $Z$ bosons are now shared by the three Higgs bosons and it may well be that the? lightest Higgs has such a weak coupling to the vector bosons that it would have been missed at LEP and will be elusive at the Tevatron. Fig. 16 shows an interesting example where the effects of $\mathrm{CP}$ violation are such that for CP-violating phases of the parameter $A_{t}$ of about $90^{\circ}$, the lightest Higgs boson canno: he detected at the Tevatron even though its mass is below $100 \mathrm{GeV}$. but the second lightest Higgs hats SM-like couplings to the $W$ and $Z$ and thus can be detected if sufficiont huninusity is provided.

\subsubsection{MSSM Higgs Searches at the LHC}

If no Higgs boson is discovered at the Tevatron, the LHC will cover the remaining unexplored regions of the $m_{A} \cdot \tan \beta$ plane, as shown in figs. 27 and 28 . That is, in the maximal mixing scenario (and probably in most regions of MSSM Higgs parameter space), at least one of the Higgs bosons is guaranteed wo be discovered at either the Tevatron and/or the LHC. A large fraction of the parameter space can be: covered by the search for a neutral CP-even Higgs boson by employing the SM Higgs search techniques. where the SM Higgs boson is replaced by $h$ or $H$ with the appropriate re-scaling of the couplings. Mureover, in some regions of the parameter space, both $h$ and $H$ can be simultaneonsly observed, and additional Higgs search techniques can be employed to discover $A$, and/or $H^{ \pm}$at the $\mathrm{LHC}$.

A CP-even Higgs boson, $\phi$, can be observed in a number of different decay modes. If $m_{4}>m_{h}$ ia then $\phi=h$ is SM-like (near the decoupling limit), whereas at large $\tan \beta$ and $m_{A}<m_{h}^{\max } .=H$ is the SM-like Higgs boson. ${ }^{28}$ It is possible to observe $\phi \rightarrow \gamma \gamma$ when $\phi$ is produced singly via gg and $V^{*} V^{* *}$ fusion, or when produced in association with $W^{ \pm}$and/or $t \bar{t}$. A second decay mode, $h \rightarrow b \bar{b}$. can be observed in $\phi t \bar{t}$ production. Finally, it may be possible to observe $\phi \rightarrow \tau^{2} \tau^{-}$when $\phi$ is produced via $V^{*} V^{*}$ fusion, where the forward jets are used to help reduce backgrounds [215]. Hencer. by using the complementarity of the various Higgs signatures described above, one can discover it over

\footnotetext{
${ }^{28}$ For $m_{A}<m_{h}^{\max }$ and moderate $\tan \beta$ values, neither CP-even Higgs boson is SW-like, althungh both Higgs massm's lit' below about $150 \mathrm{GeV}$ and will appear (albeit with reduced couplings to $V V$ ) in the Higgs searches described above.
} 

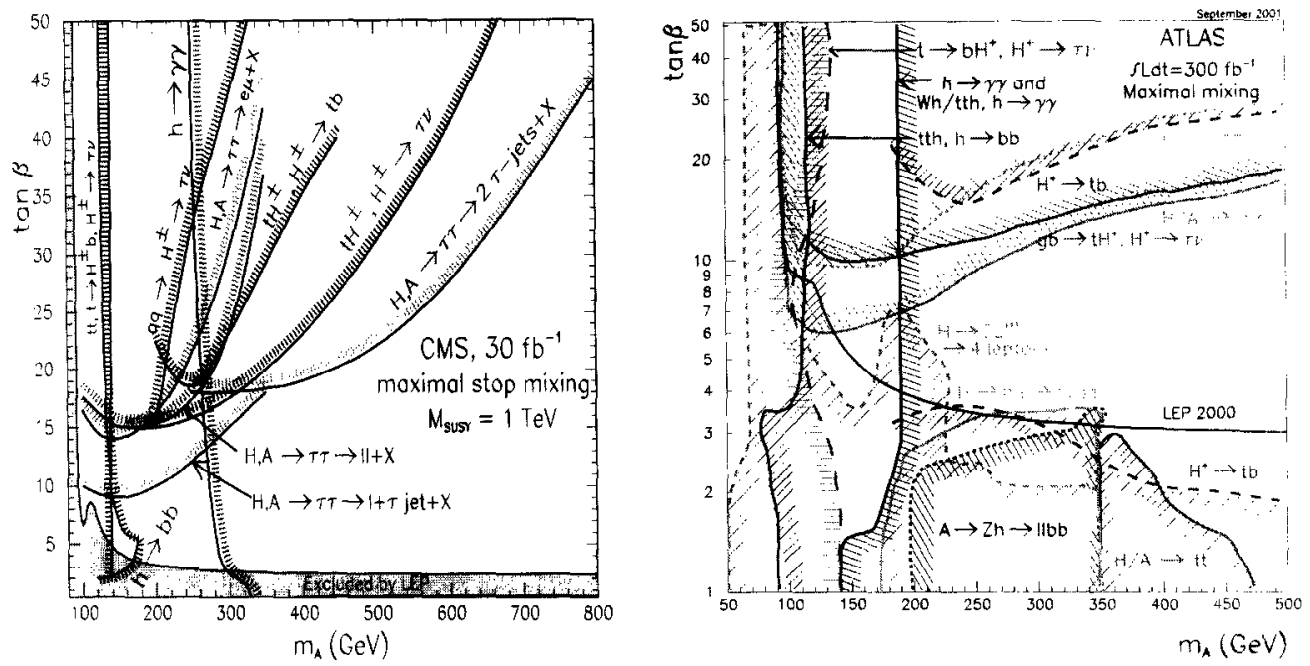

Figure 27: (a) $\check{\sigma} \sigma$ discovery contours for MSSM Higgs boson detection in various channels in the $m_{A}-\tan i s$ plane, in the maximal mixing scenario, assuming an integrated luminosity of $L=30 \mathrm{fb}^{-1}$ for the CMS detector [218]. (b) As in (a), but for an integrated luminosity of $L=300 \mathrm{fb}^{-1}$ for the ATLAS detector [222].

nearly the entire MSSM parameter space, given sufficient integrated luminosity. ${ }^{29}$ In order to illustrate the complementarity of the $\gamma \gamma$ and $b \bar{b}$ decay modes, we exhibit in fig. 29 the regions of MSSM Higgs parametcr space that can be covered for the two benchmark scenarios of MSSM parameters described in Section 3.5.2. The behavior illustrated in this figure can be understood by noting that the $\phi b \vec{b}$ coupling can be significantly suppressed (or enhanced), depending on the impact of the radiative corrections discussed in Section 3.3. As a result, the branching ratio for $\phi \rightarrow \gamma \gamma$ is correspondingly larger (or smaller), with obvious implications for the $\phi \rightarrow b \bar{b}$ and $\phi \rightarrow \gamma \gamma$ searches.

We next focus on the potential for observing the heavier Higgs states $\left(H^{ \pm}, A\right.$ and $H$ ). A number of recent studies $[74,75,218-221]$ show that the following modes will be effective in searching for the heavier MSSM Higgs bosons. For the heavy neutral Higgs bosons, the most relevant decay signatures

\footnotetext{
${ }^{29}$ One must still demonstrate that it is possible at the LHC to discover the lightest CP-even Higgs boson, even if its branching ratios into $b \bar{b}$ and/or $\gamma \gamma$ are significantly suppressed (either due to the effects of radiative corrections or due to the existence of a significant branching fraction into invisible modes). Such suppressions can occur in regions of the MSSM parameter space not yet considered by the LHC Higgs search simulations.
} 


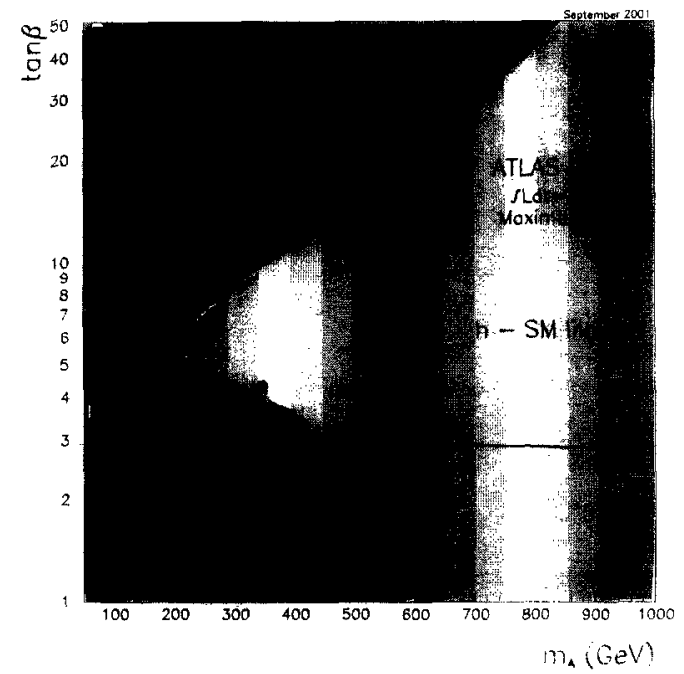

Figure 28: Regions in the $m_{A} \tan \beta$ plane in the maximal mixing scenario in which up to four Higgs boson states of the MSSM can be discovered at the LHC with $300 \mathrm{fb}^{-1}$ of data, based on a simulation that combines data from the ATLAS and CMS detectors. Taken from ref. [87].

are: $A, h \rightarrow \tau^{+} \tau^{-}$(where the $\tau$ is detected either via its leptonic or hadronic deray) and $A, h \rightarrow h^{*} h^{\prime}$. which yield promising signals if $\tan B$ is large. The $\tau^{+} T^{-}$channel provides the latgest discovery erath in the heavy Higgs mass. Other possible neutral Higgs decays; $A$. $H \rightarrow \| l: H \rightarrow Z Z^{*} \rightarrow 4 t: H \rightarrow h$ h and $A \rightarrow Z h$ are significant in regions of the parameter space that are (nearly) ruled out by the LEP Higgs search. For the charged Higgs boson, we must again consider whether $H^{ \pm}$can be produced in (on-shell) top-quark decays. If this decay is forbidden, the positively charged Higgs boson will be pruduced primarily by $g b \rightarrow H^{+} t$ (see Section 3.5.1). In either case. the observation of the chared Iliggs boson is possible if $\tan \beta \gg 1$ or $\tan \beta \lesssim \mathcal{O}(1)$ [221]. For lange tan $\}^{2}$ the derays $I^{+}+\ldots$ and $t \bar{b}$ (if kinematically allowed) provide the most favorable signatures. In particular. the ot" decay mode, followed by the hadronic decay of the $\tau$ provides the largest cliscorry reach for large $m_{H}$. . The: ultimate charged Higgs mass reach can depend significantly on the rhoice of MSSM paraneter's that control the radiative corrections to the Higgs-bottom quark Yukawa coupling [196] [ser. r.y. ec. (T)

Putting all of the above results together, it may be possible at the LHC: to either exclude the cutire $m_{d} \tan i \beta$ plane (thereby eliminating the MSSM Higgs sector as a viable model) or ardicu a 

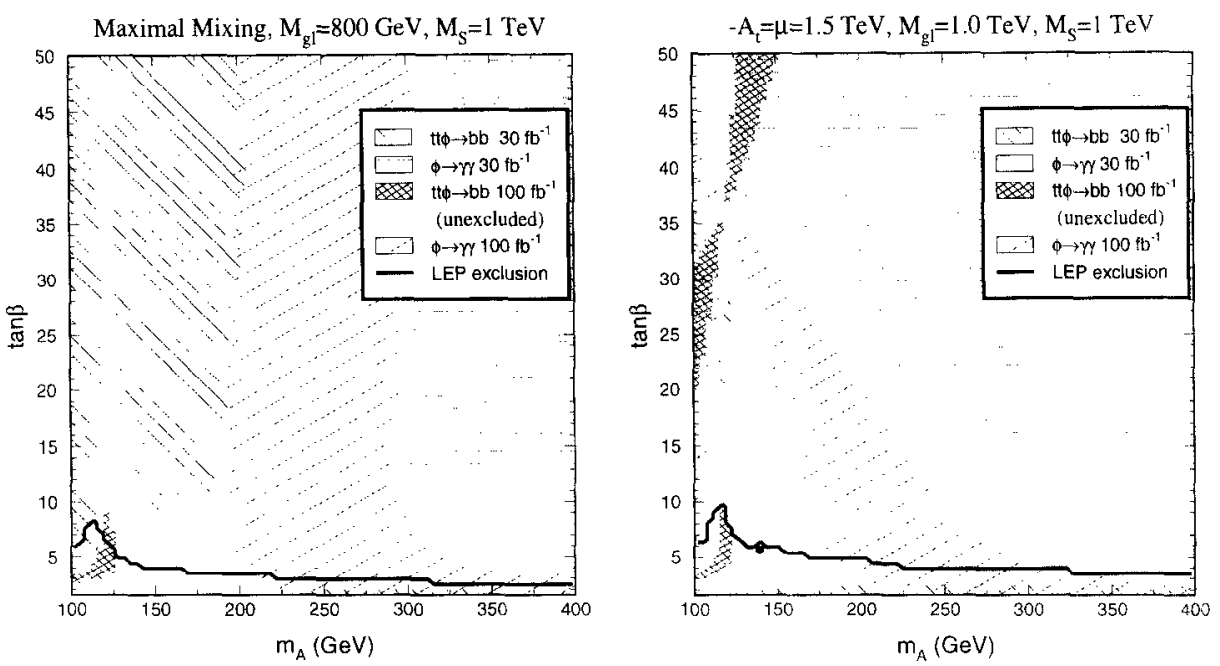

Figure 29: Complementarity between the LHC searches for the decay modes $\phi \rightarrow b \bar{b}$ and $\phi \rightarrow \gamma \gamma$, where $\phi=h$ or $H$ corresponds to the CP-even Higgs boson with the larger coupling to $V V$. Two different choices of MSSM parameters are exhibited: (a) the maximal mixing scenario and (b) the suppressed $V b \rightarrow V b \bar{b}$ production scenario. In both cases, the region corresponding to $5 \sigma$ discovery of $\phi \rightarrow \gamma \gamma$ with $30 \mathrm{fb}^{-1}$ of data is shaded with parallel horizontal lines. With $100 \mathrm{fb}^{-1}$ of data, these regions expand to include areas shaded with diagonal parallel lines with positive slope. The region corresponding to $5 \sigma$ discovery of $t \bar{t} \phi \rightarrow t \bar{t} b \bar{b}$ with $30 \mathrm{fb}^{-1}$ of data is shaded with diagonal parallel lines with negative slope. With $100 \mathrm{fb}^{-1}$ of data, these regions expand to include the entire $m_{A} \tan \beta$ plane excluding the blue cross-hatched region. The "unexcluded" region (where no discovery of $t \bar{t} \phi \rightarrow t \bar{t} b \bar{b}$ is possible) occupies a small region at low $\tan B$ and $m_{A}$ in both (a) and (b). In addition, in case (b), the excluded region also includes the two narrow wedge regions at large $\tan \beta$ and low $m_{A}$. Taken from ref. [223].

$5 \sigma$ discovery of at least one of the MSSM Higgs bosons, independently of the value of $\tan \beta$ and $m_{A}$. For example, Fig. 27 shows what can be achieved by the CMS detector with $30 \mathrm{fb}^{-1}$ [218] and by the ATLAS detector with $300 \mathrm{fb}^{-1}$ [222], assuming the maximal mixing scenario. Note that over a significant fraction of the MSSM Higgs parameter space, at least two Higgs bosons can be observed as shown in fig. 28. Nevertheless, there is still a sizable wedge-shaped region at moderate values of $\tan B$ opening up from about $m_{A}=200 \mathrm{GeV}$ to higher values in which the heavier Higgs bosons cannot be discovered at the LHC. In this parameter regime, only the lightest CP-even Higgs boson can be discovered, and its properties are nearly indistinguishable from those of the SM Higgs boson. Precision measurements of Higgs branching ratios and other properties will be required in order to detect deviations from SM Higgs predictions and demonstrate the existence of a non-minimal Higgs sector 
Finally, we noted at the end of Section 3.5.3 that CP-violating effects in the Higgs sertur ant modify the usual CP-conserving Higgs phenomenology. As a result, the LHC discovery reach ut varium Higgs channels discussed above may be altered in a significant way. It is therefore essential to nuk complementary measurements in as many Higgs channels as possible in order to cover the most gemeral MSSM parameter space [223].

\subsection{MSSM Higgs Boson Searches at the LC}

The main production mechanisms for the MSSM Higgs bosons are [2:4]

$$
\begin{aligned}
& \text { (i) } e^{+} e^{-} \rightarrow Z h, Z H \text { via Higgs-strahlumg: }
\end{aligned}
$$

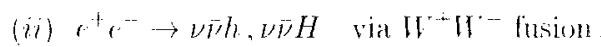

$$
\begin{aligned}
& \text { (iii) } e^{+}+\rightarrow h+A, H A \text { via s-channel } Z \text { exchange. } \\
& \text { (ii) } \theta \rightarrow H^{+} H \text { via s-channel y. \% exchange. }
\end{aligned}
$$

As in the SM Higgs search, process $(i)$ followed by $Z \rightarrow t^{+} l^{-}$allows the Higgs boson recoiling against the $Z$ to be reconstructed, independently of the Higgs decay channel. Thus. $h[$ or $H$ if $\sin (\beta-\alpha) \ll 1]$ (an be discovered at the LC even if it has a large branching fraction intu invisible modes. ${ }^{30}$

Processes (i) and (iii) are complementary to each other as a consequence of unitarity sum rules fol

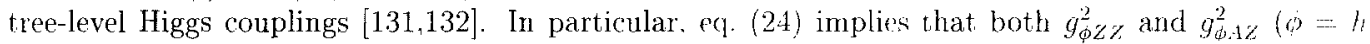
or $H$ ) cannot simultaneously vanish. If $m_{1} \lesssim m_{h}^{\max }$, then all the MSSM Higgs boson states have mass below $150 \mathrm{GeV}$, and can be cleanly reconstructed at the LC (with $\sqrt{s} \geq 350$ GeV) via the four production mechanisms listed above [92]. On the other hand, when $m_{1} z 200$ GeV. one finds that $m_{A} \sim m_{H} \sim m_{H \pm}$ and $g_{H Z Z} \sim g_{h A Z} \sim 0$, and the couplings of $h$ are nearly identical to those of $h_{\mathrm{s} M}$ as a consequence of the decoupling limit. Since $m_{h} \leqslant 135$ (iell the LC with a center-of-mass energy of $300 \mathrm{GeV}$ is more than sufficient to observe the he [via processes (i) and (ii)] and thus cower the entire MSSM parameter space with certainty. Noreover, the rossisentions for $H Z$. Heis and $h . t$

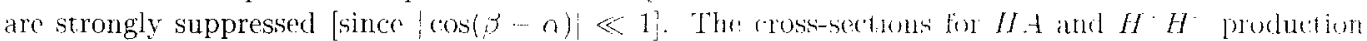
are unsuppressed if kinematically allowed. ${ }^{31}$ That is, the heavy Higgs bosons. $H$. A and $H^{ \pm}$can only be observed in pair production processes where both Higgs states are heavy (and the minimmm required is somewhat above $\left.2 m_{1}\right)$. These features are evident in fig. 30, which clepicts cross-sections for Higgs-strahlung [process (i)] and associated Higgs pair production [processes (iii) and (iv)] as ib function of the corresponding Higgs mass for two different choices of $\sqrt{s}$ and tall $\beta$. The cross-section

\footnotetext{
${ }^{30}$ The processes $e^{+} e^{-} \rightarrow e^{+} e^{-} h, e^{\frac{1}{2}} e^{-H}$ (via $Z Z$ fusion) alsu allow for Higgs detection, independentiy of its decily channel, by reconstruction of the Higgs boson recoiling against the final state, ${ }_{t}$. pair. However, the $Z Z$ fusion ratre are an order of magnitude smaller than the corresponding $W^{+} W^{-}$fusion rates [proress $(i i)$,

${ }^{31}$ Due to the $p$-wave suppression at threshold. the $H A$ and $H^{+} H^{-}$cross-sections fall off rapidly as the corresponting Hirgs masses approach $\sqrt{s} / 2$.
} 

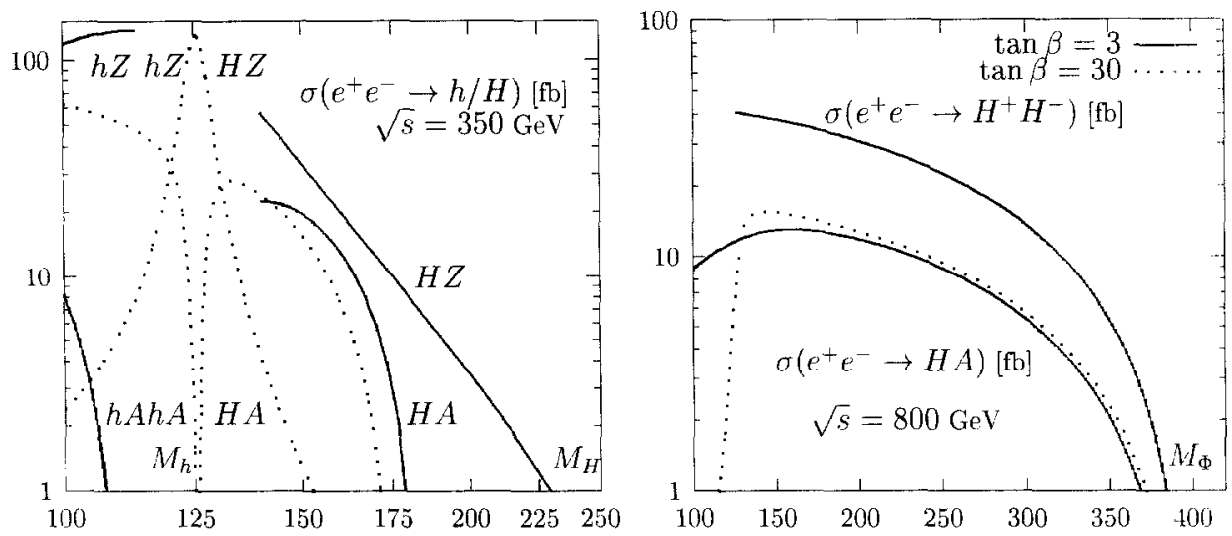

Figure 30: MSSM Higgs boson production rates at the LC for two choices of $\tan \beta=3$ (solid) and 30 (dotted) for (a) $\sqrt{s}=350 \mathrm{GeV}$ as a function of the mass of the produced CP-even neutral Higgs boson (either $h$ or $H$ ); and for (b) $\sqrt{s}=800 \mathrm{GeV}$ as a function of $m_{H^{ \pm}}$and $m_{A}$, respectively. Taken from ref. [225].

for Higgs production via $W^{+} W^{-}$fusion [process (ii)] is not shown. The $\nu \bar{\nu} \phi$ production cross-section is suppressed relative to the corresponding SM cross-section (shown in fig. 9) by a factor of $\sin ^{2}(\beta-\alpha)$ $\left[\cos ^{2}(\beta-\alpha)\right]$ for $\phi=h[\phi=H]$.

In addition to $H^{+} H^{-}$production, there are a number of mechanisms in which the charged Higgs boson is singly produced. Charged Higgs bosons can be produced in top decays via $t \rightarrow b+H^{+}$if $m_{H^{ \pm}}<m_{t}-m_{b}$, as discussed previously in Section 3.5.1. The process $e^{+} e^{-} \rightarrow W^{+} I I^{\mp}$, which arises at une-loop [226,227], allows for the possibility of producing a charged Higgs boson with $m_{H^{ \pm}}>\sqrt{s} / 2$, when $H^{+} H^{-}$production is kinematically forbidden. With favorable MSSM parameters and moderate values of $\tan \beta$, more than ten $W^{ \pm} H^{\mp}$ events can be produced at the LC for $m_{H^{ \pm}} \lesssim 350 \mathrm{GeV} V$ with $\sqrt{s}=500 \mathrm{GeV}$ and $500 \mathrm{fb}^{-1}$ of data, or for $m_{H^{ \pm}} \lesssim 600 \mathrm{GeV}$ with $\sqrt{s}=1 \mathrm{TeV}$ and $1 \mathrm{ab}^{-1}$ [22 $\left.\mathrm{T}\right]$. Other single charged Higgs production mechanismis include $t \bar{b} H^{-} / \bar{t} b H^{+}$production [101], $\tau^{+} \nu H^{-} / \tau^{-} \bar{\nu} H^{+}$ production [228], and a variety of processes in which $H^{ \pm}$is produced in association with a one or two other gauge and/or Higgs bosons [229].

The heavier Higgs states could lie beyond the discovery reach of the $\mathrm{LC}(\sqrt{s} \leq 1 \mathrm{TeV}$ ) and the LHC [cf. fig. 28]. In this case, the precision measurements of the $h$ decay branching ratios and couplings achievable at the $\mathrm{LC}$ are critical for distinguishing between $h_{\mathrm{SM}}$ and $h$ of a non minimal Higgs sector with properties close to that of the SM Higgs boson. To illustrate the challenge of probing the decoupling linit, suppose that $m_{A}>\sqrt{s} / 2$ so that only the light Higgs boson, $h$, can be observed directly at the 
(a) Maximal Mixing Scenario

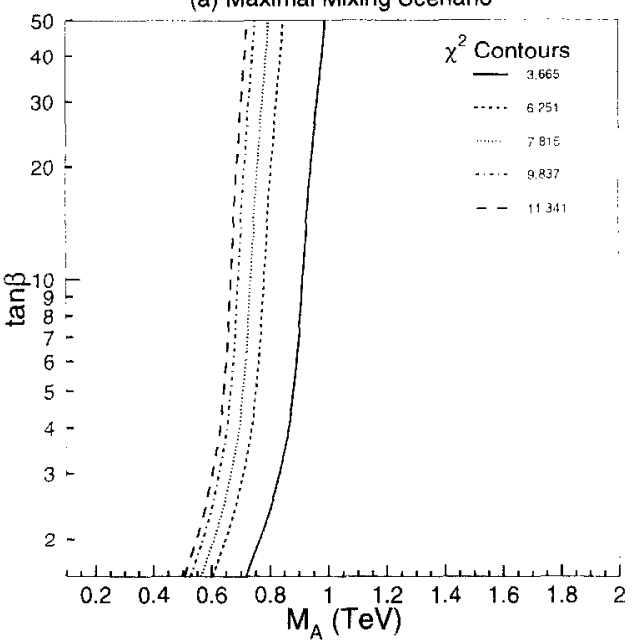

(b) $A=-\mu=1.2 \mathrm{TeV}, M_{g}=.5 \mathrm{TeV}$

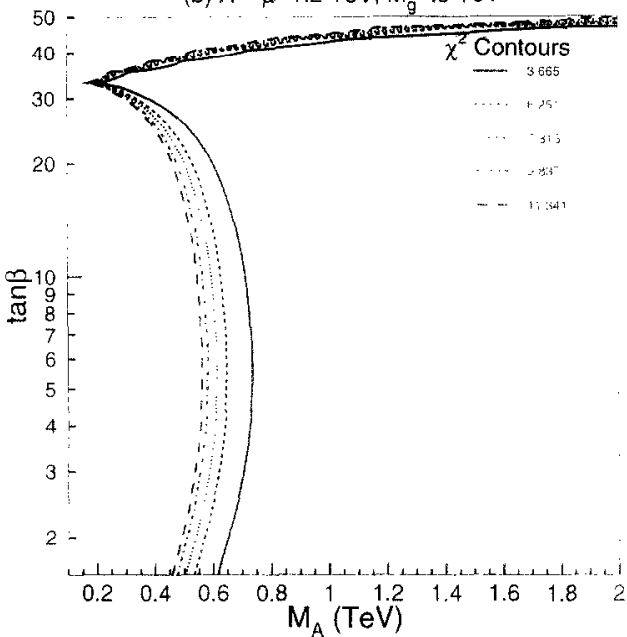

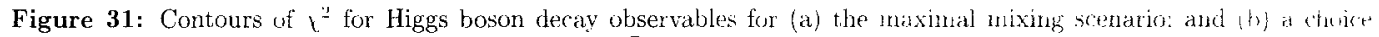
of MSSM parameters for which the loop-corrected $h b \bar{b}$ coupling is suppressud (rolative to the corresjonding tree-lewal coupling) at large $\tan \beta$ and low $m_{A}$. These results are based on Higgs partial width measurements anticipated at thi $L(:$ (shown in Table 1) with $\sqrt{s}=500 \mathrm{GeV}$ and an integrated luminosity of $500 \mathrm{fb}^{-1}$. The contours correspond to $68.90,95$. 98 and $99 \%$ confidence levels (right to left) for the observables $g_{h u b}^{2}, g_{h++}^{2}$, and $g_{h, y,}^{2}$. See ref. [112] for additiona] details.

LC. In this case, the fractional deviation of the couplings of $h$ relative to those of the SM Higgs boson scales as $m_{Z}^{2} / m_{A}^{2}$. Thus, if precision measurements reveal a non-zero deviation, one conld in principle: derive a constraint (e.g., upper and lower bounds) on the heavy Higgs masses of the model. In the MSSM. the constraint is sensitive to the MSSM parameters that control the radiative corrections to the Higgs couplings. This is illustrated in fig. 31. where the constraints on $m_{\mathrm{A}}$ are derived for two different sets of MSSM parameter choices [112]. Here, a simulation of a global fit of measured h.bb, hTT and hgy couplings is made (based on the results of Table 1) and $x$ " contours are plotted indicaling the? constraints in the $m_{A}$-tan $\beta$ plane, assuming a deviation from SM Higgs boson couplings is seen. ${ }^{32}$ In the maximal mixing scenario shown in fig. 31(a), the constraints on $m_{A}$ are significant and rather insensitive to the value of $\tan \beta$. Similar results, in which deviations of $\mathrm{BR}(h \rightarrow b \bar{b}) / \mathrm{BR}\left(h \rightarrow \tau^{+} \tau^{-}\right)$from the

\footnotetext{
${ }^{32}$ These results are similar to those of ref. $[107]$, in which $m h_{\mathrm{L}}=120 \mathrm{GeV}$ and an integrated LC luminosty of loun hi were assumed. The upper bound on $m_{A}$ as a function of tan $b$ was determinal in which $68 \% .90 \%$ and $95 \%$ of the MSSM parameter space, respectively, yield $h$ branching ratio predictions that differ from those of the SM at the $95 \%$ CL. However, the MSSM parameter regimes in which the sensitivity to $m_{A}$ are weakest were not identified in ref. [107].
} 
SM prediction yield limits on the allowed values of $m_{A}$ as a function of $\tan \beta$, have been obtained in ref. [230]. However in some cases, as shown in fig. 31(b), a region of $\tan \beta$ may yield almost no constraint on $m_{A}$. This is due to the phenomenon of $m_{A}$-independent decoupling noted below eq. (54), in which $\cos (\beta-\alpha)$ [which controls the departure from the decoupling limit] vanishes at a particular value of $\tan \beta$ independently of the value of $m_{A}$. Thus, one cannot extract a fully model-independent upper bound on the value of $m_{A}$ (beyond what can be deduced if no direct $A$ production is observed at the LC). Of course, after supersymmetric particles are discovered, information about the MSSM spectrum (an be used to obtain a more stringent bound on $m_{A}$, using the techniques described above.

The $e^{+} e^{-}$linear collider running in the $\gamma \gamma$ collider mode presents additional opportunities for the struly of the MSSM Higgs sector. Resonance production $\gamma \gamma \rightarrow H$ and $A$ can be used to extend the reach in Higgs masses beyond the limit set by $H . A$ pair production in the $e^{+} e^{-}$mode $[231,123,124]$. Typically, one can probe the heavy Higgs masses out to $m_{A} \sim 0.8 \sqrt{s}$ (where $\sqrt{s}$ is the center of mass energy of the LC). This expands the MSSM Higgs discovery reach further into regions of the $m_{A^{-}} \tan \beta$ parameter space for which the LHC is not sensitive in general (the so-called "blind wedge" of large $m_{A}$ and moderate values of $\tan \beta$ seen in fig. 28).

As noted above, at least one Higgs boson must be observable at the LC in the MSSM. In non-minimal supersymmetric models, additional Higgs bosons appear in the spectrum, and the "no-lose" theorem of the MSSM must be reconsidered. For example, in the non-minimal supersymmetric extension of the Standard Model (the so-called NMSSM where a Higgs singlet is added to the model [232]), the lightest Higgs boson decouples from the $Z$ boson if its wave function is dominated by the Higgs singlet component. However, in this case the second lightest neutral CP-even Higgs boson usually plays the role of $h$ of the MSSM. That is, the mass of the second lightest Higgs boson is typically below $150 \mathrm{GeV}$ with significant couplings to $Z Z$, so that it can be produced by the Higgs-strahlung process with an otservable cross-section $[233,158]$. If the second lightest Higgs boson also decouples from the $Z$. then the third lightest Higgs boson will play the role of $h$ of the MSSM for which the observation is ensured, and $s o$ on. Even in bizarre scenarios where all the neutral Higgs boson share equally in the coupling to $Z Z$ (with the sum of all squared couplings constrained to equal the square of the $h_{\mathrm{SM}} Z Z$ coupling $[131,132]$ ), the "no-lose" theorem still applies--Higgs production at the LC must be observable [234]. In contrast, despite significant progress, there is no complete guarantee that at least one Higgs boson of the NMSSM must be discovered at the LHC for all choices of the model parameters [235].

One of the key parameters of the MSSM Higgs sector is the value of the ratio of Higgs vacuum expectation values, $\tan \beta$. In addition to providing information about the structure of the non-minimal Higgs sector, the measurement of this parameter also provides an important check of supersymmetric structure, since this parameter also enters the chargino, neutralino and third generation squark mass matrices and couplings. Thus, $\tan \beta$ can be measured independently using supersymmetric processes and compared to the value obtained from studying the Higgs sector. Near the decoupling limit, the properties of $h$ are almost indistinguishable from those of $h_{\mathrm{SM}}$. and thus no information can be extracted on the value of $\tan \beta$. However, the properties of the heavier Higgs bosons are $\tan \beta$-dependent. Far 
from the decoupling limit, all Higgs bosons of the MSSM will be observable at the LC and rxhibit strong tan $\beta$-dependence in their couplings. Thus, to extract a value of tan $\beta$ from Higgs processes, ont must observe the effects of the heavier Higgs bosons of the MSSM at the LC:

The ultimate accuracy of the $\tan \beta$ measurement at the LC depends on the value of tan $;$. In Ref. [236], it is argued that one must use a number of processes, including $b b b \dot{b}$ final states arising from $b \vec{b} H, b \bar{b} A$, and $H A$ production, and $t \bar{t} b \bar{b}$ final states arising from $t \bar{b} H^{+}, b \vec{b} H^{-}$and $H^{+} H^{-}$production. One subtlety that arises here is that in certain processes, the determination of $\tan \beta$ may be sensitive to loop corrections that depend on the values of other supersymmetric parameters. One must settle on a consistent definition of $\tan \beta$ when loop corrections are included [237]. A comprehensive analysis uf the extrartion of $\tan \beta$ from collider data, which incorporates loop rffects has not yet been given

The study of the properties of the heavier MSSM Higgs bosons (mass. width, branching ratios. quantum numbers, etc.) provides a number of additional challenges. For example, in the ahsencre of CP-violation, the heavy CP-even and CP-odd Higgs bosons, $H$ and $A$. ale expected to be nearly masdegenerate. Their CP quantum numbers and their separation can be investigated at the same timu in the on collider mode of the LC. If the polarization states of the two incoming linearly-polarized photons are parallel [perpendicular] then only the CP-even Higgs boson $H$ [CP-odd Higgs boson A] will he produced [238]. Thus, the determination of the Higg; boson CP quantum numbers and the separation of the lwo different states can be achieved. In the case of a CP-violating Higgs sectol: the observation and neeasurement of Higgs boson properties become much more challenging. The on collider can proride new opportunities to test the nature of the couplings of the Higgs noutral cigenstates (with indefirite CP quantum numbers) to gauge bosons and fermions [124,239].

Finally, once the heavy Higgs spectrum is revealed, one would like to recunstru't the two-figgsdoublet scalar potential [240]. This is not likely to be accomplished at a first generation LC, although une can make a start if the heavy Higgs masses are not too large. To probe aspects of the Higgs potential onc must observe multiple Higgs production in order to extract the Higgs self-couplings $[113,114,240,241$. Lltimately, such a program would require an LC with very high energy and luminosity such as CLIC

\section{Conclusions}

The physical origin of electroweak symmetry breaking is not yet knuwn. In all theoretical approachus and models, the dynamics of electroweak symmetry breaking must be revealed at the TeV-scale or below. This energy scale will be thoroughly explored by hadron colliders, starting with the Tevatron and followed later in this decade by the LHC. Even though the varions theoretical alternatives can only be confirmed or ruled out by future collider experiments, a straightforward interpretation of the "lectroweak precision data suggests that electroweak symmetry breaking dynamics is weakly-rouplect. and a Higgs boson with mass between 100 and $200 \mathrm{GeV}$ must exist. With the supersymmetric extension of the Standard Model, this interpretation opens the route to grand unification of all the fundamental 
forces, with the eventual incorporation of gravity in particle physics.

In this review we have summarized the theoretical properties of the Standard Model Higgs boson and the Higgs bosons of the MSSM, and surveyed the search strategies for discovering the Higgs boson at hadron and lepton colliders. We have assessed the Higgs boson discovery reach of present and future colliders, and described methods for measuring the various Higgs boson properties (mass, width, CP quantum numbers, branching ratios and coupling strengths).

The ubservation of a Higgs boson in the theoretically preferred mass range below $200 \mathrm{GeV}$ may be possible at the Tevatron, whereas experiments at the LHC can discover the SM Higgs boson over the full Higgs mass range up to $1 \mathrm{TeV}$. The Tevatron can also extend the LEP search for Higgs bosons of the MSSM by either discovering the lightest CP-even MSSM Higgs boson, $h$ (or in some special cases discovering additional Higgs scalars of the model), or by further constraining the MSSM Higgs parameter space. The LHC is scnsitive to nearly the entire MSSM Higgs parameter space, in which either $h$ alone can be discovered or multiple Higgs states can be observed. A program of Higgs measurements will be initiated at the LHC to measure Higgs partial widths with an accuracy in the range of $10-30 \%$.

The discovery of the Higgs boson at the Tevatron and/or the LHC is a crucial first step. The measurement of Higgs properties at the LHC will begin to test the dynamics of electroweak symmetry breaking. However, a high-luminosity $e^{+} e^{-}$linear collider, now under development, is needed for a systematic program of precision Higgs measurements. For example, depending on the value of the Higgs mass, branching ratios and Higgs couplings can be determined in some cases at the level of a few percent. In this way, one can extract the properties of the Higgs sector in a comprehensive way, and cstablish (or refute) the existence of scalar sector dynamics as the mechanism responsible for generating the masses of the fundamental particles.

\section{Acknowledgments}

We would like to thank Ulrich Baur, Sally Dawson, Jack Gunion, Heather Logan, Carlos Wagner, Georg Weiglein, Scott Willenbrock and Peter Zerwas for enlightening discussions. We are especially grateful to Steve Mrenna and Michael Spira for many useful suggestions and for their dedicated work in providing a number of plots shown in this review. Finally, much of this work would not have been possible without the collective wisdom gathered from our colleagues of the 1998 Tevatron Higgs Working Group and the 2001 Snowmass Electroweak Symmetry Breaking Working Group.

Fermilab is operated by Universities Research Association, under contract no. DE-AC02-76CH03000 with the U.S. Department of Energy. H.E.H. is supported in part by the U.S. Department of Energy under grant no. DE-FG03-92ER40689. 


\section{References}

[1] P.W. Higgs, Phys. Rev. Lett. 12 (1964) 132; Phys. Rev. 145 (1966) 1156; F. Englert and R. Brout, Phys. Rev. Lett. 13 (1964) 321; G.S. Guralnik, C.R. Hagen, and T.W.B. Kibble, Phys Rcv. Lett. 13 (1964) 585.

[2] J.F. Gunion, H.E. Haber, G. Kane and S. Dawson, The Higgs Hunter's Guide (Persens Publishing, Cambridge, MA, 1990).

[3] H.P. Nilles, Phys. Reports 110 (1984) 1; H.E. Haber and G.L. Kanr, Phys. Reports 117 (1985) it S.P. Martin, hep-ph/9709356; P. Fayet, Nucl. Phys. B (Proc: Suppl.) $101(2001) 81$

[1] G. 't Hooft, in Recent Developments in Gauge Theories. Proceedings of the NAT() Advanced Summer Institute, Cargèse, 1979, edited by G. 't Hooft et al. (Ple'mum. Now York 1980! p. 1355: M. Veltman, Act. Phys. Pol. B12 (1981) 437.

[5] E. Witten, Nucl. Phys. B188 (1981) 513; R.K. Kaul, Phys. Lott. 109B. 19 (1982): Pramana 19. 183 (1982); L. Susskind, Phys. Reports 104 (1984) 181.

[6] K. Inoue, A. Kakuto, H. Komatsu, and S. Takeshita, Prog. Theor. Phys. 68 (1982) 927 [L: 70 (1983) 330]; 71 (1984) 413; R. Flores and M. Sher, Ann. of Phys. 148 (1983) 95; J.F. Gunion and H.E. Haber, Nucl. Phys. B272 (1986) 1 [E: B402 (1993) 567].

[7] S. Weinberg, Physs. Rerv. D13 (1979) 974; D19 (1979) 1277: L. Susskind, Phys. Rerv. D20 (1979) 2619; E. Farhi and L. Susskind. Phys. Reports 74 (1981) 277: R.K. Kanl Rer. Mod. Phw 55 (1983) 449: C.T. Hill and E.H. Simmons. FERMI-PUB-(12/045-T [hep-ph/0203079]. 1. w published in Phys. Reports.

[8] N. Arkani-Hamed, A.G. Cohen and H. Georgi, Phys. Lett. B513 (2001) 232; N. Arkani-Hamed. A.G. Cohen, E. Katz and A.E. Nelson. JHEP 0207 (2002) 034. N. Arkani-Hamert A G Cohen. T. Gregoire and J.G. Wacker, JHEP 0208 (2002) 020. N. Arkani-Hamed. A.G. Cohen. E. Katz. A.E. Nelson, T. Gregoire and J.G. Wacker. JHEP 0208 (2002) 021; I. Low. W. Skiba and D. Smith. Phys. Rer. D66 (2002) 072001.

(9) I. Antoniadis, Phys. Lett. B246 (1990) 377; N. Arkani-Hamed, S. Dimopoulus and G.R. Dvali, Phys. Lett. B429 (1998) 263; L. Randall and R. Sundrum, Phrs. Rev. Lett. 83 (1999) 3370. For a recent review, see V.A. Rubakov, Phys. Usp. 44 (2001) 871.

[10] D. Abbaneo et al. [LEP Electroweak Working Group] and A. Chou et al. [SLD Heary Flitvor and Electroweak Groups], LEPEWWG/2002-01 (8 May 2002) and additional updates at http://lepewwg . web. cern. ch/LEPEWWG/. 
[11] ALEPH, DELPHI, L3 and OPAL Collaborations, The LEP working group for Higgs boson searches, LHWG Note 2002-01 (July 2002), and additional updates at http://lephiggs . web . cern. ch/LEPHIGGS/www/welcome.html.

[12] J. Erler, Phys. Rev. D63 (2001) 071301; talk given at 50 Years of Electroweak Physics, A Symposium in Honor of Professor Alberto Sirlin's 70th Birthday, New York, NY, 27-28 October 2000 [hep-ph/0102143].

[13] R. Barbieri and A. Strumia, Phys. Lett. B462 (1999) 144; R.S. Chivukula and N. Evans, Phys. Lett. B464 (1999) 244; L.J. Hall and C. Kolda, Phys. Lett. B459 (1999) 213; B.A. Kniehl and A. Sirlin, Eur. Phys. J. C16 (2000) 635; J.A. Bagger, A.F. Falk and M. Swartz, Phys. Rev. Lett. $84(2000) 1385$.

[14] C. Kolda and H. Murayama, JHEP 0007 (2000) 035.

15] R. Casalbuoni, S. De Curtis, D. Dominici, R. Gatto and M. Grazzini, Phys. Lett. B435 (1998) 396; R.S. Chivukula, B.A. Dobrescu, H. Georgi and C.T. Hill, Phys. Rev. D59 (1999) 075003; T.G. Rizzo and J.D. Wells, Phys. Rev. D61 (2000) 016007; R.S. Chivukula, C. Hoelbling and N. Evans, Phys. Rev. Lett. 85 (2000) 511; P. Chankowski, T. Farris, B. Grzadkowski, J.F. Gunion, J. Kalinowski and M. Krawczyk, Phys. Lett. B496 (2000) 195; H.C. Cheng, B.A. Dobrescu and C.T. Hill, Nucl. Phys. B589 (2000) 249; H.J. He, N. Polonsky and S.F. Su, Phys. Rev. D64 (2001) 053004; M.E. Peskin and J.D. Wells, Phys. Rev. D64 (2001) 093003; H.J. He, C.T. Hill and T.M. Tait, Phys. Rev. D65 (2002) 055006; V.A. Novikov, hep-ph/0205320.

[16] M.E. Peskin and T. Takeuchi, Phys. Rev. Lett. 65 (1990) 964, Phys. Rev. D46 (1992) 381.

17. G. Mtarelli, F. Caravaglios, G.F. Giudice, P. Gambino and G. Ridolfi, JIIEP 0106 (2001) 018; M.S. Chanowitz, Phys. Rev. Lett. 87 (2001) 231802; Phys. Rev. D66 (2002) 073002; D. Choudhury, T.M. Tait and C.E.M. Wagner, Phys. Rev. D65 (2002) 053002; S. Davidson, S. Forte, P. Gambino, N. Rius and A. Strumia, JHEP 0202 (2002) 037; W. Loinaz, N. Okamura, T. Takeuchi and L.C. Wijewardhana, hep-ph/0210193.

[18] L.E. Ibáñez and G.G. Ross, Phys. Lett. B105 (1981) 439; S. Dimopoulos, S. Raby, F. Wilczek, Phys. Rev. D24 (1981) 1681; M.B. Einhorn and D.R.T. Jones, Nucl. Phys. B196 (1982) 475; W.J. Marciano and G. Senjanovic, Phys. Rev. D25 (1982) 3092.

[19 J. Ellis, S. Kelley and D.V. Nanopoulos, Phys. Lett. B249 (1990) 441; P. Langacker and M. Luo, Phys. Rev. D44 (1991) 817; L. Amaldi, W. de Boer and H. Fürstenau, Phys. Lett. B260 (1991) 447; P. Langacker and N. Polonsky, Phys. Rev. D52 (1995) 3081; S. Pokorski, Act. Phys. Pol. B30 (1999) 1759. For a recent review, see: R.N. Mohapatra, in Particle Physics 1999, Proceedings 
of the ICTP Summer School in Particle Physics, Trieste. Italy, 21 June-9 July, 1999 , edited by G. Senjanovic and A.Yu. Smirnov. (World Scientific, Singapore, 2000) pp. 336-394.

[20] T. Hambye and K. Riesselmann, Phys. R.ev. D55 (1997) 725\%.

[21] See, e.g., G. Altarelli and G. Isidori, Phys. Lett. B337 (1994) 141; J.A. Casas, J.R. Espinosd and M. Quirós, Phys. Lett. B342 (1995) 171; B382 (1996) 37 .

[22] K. Riesselmann, DESY'-97-222 (1997) [hep-ph/9711456].

[23] G. Isidori, G. Ridolfi and A. Strumia, Nucl. Phys. B609 (2001) 387.

[24] J.M. Cornwall, D.N. Levin and G. Tiktopoulos. Phys. Rev. Lett. 30 (1973) 1268; Phys. Rer. D10 (1974) 1145; C.H. Llewellyn Smith. Phys. Lett. 46B (1973) 233.

[25] B.W. Lee, C. Quigg and H.B. Thacker, Phws. Rev. D16 (1977) 1519.

[26] B.A. Kniehl, Phys. Reports 240 (1994) 211

[27] A. Djouadi, J. Kalinowski and M. Spira. Comp. Phrs. Commun. 108 (1998) 56. The HDE(A)

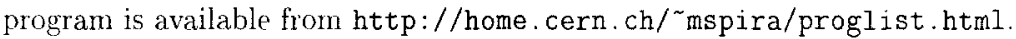

[28] E. Braaten and J.P. Leveille, Phys. Rer. D22 (1980) 715: N. Sakai. Phys. Rev. D22 (1980) 2220; T. Inami and T. Kubota, Nucl. Phys. B179 (1981) 171; S.G. Gorishniī, A.L. Kataey and S.A. Larin, Sov. J. Nucl. Phys. 40 (1984) 329 [Yad. Fiz. 40 (1984) 517]; M. Drees and K. Hikasa, Phys. Lett. B240 (1990) 455 [E: B262 (1991) 497].

[29] A. Djouadi, M. Spira and P.M. Zerwas, Z. Phys. C70 (1996) 42-.

[30] D.Ýu. Bardin, B.M. Vilenskin and P. Kh. Khristov, Sor. J. Nucl Phys. 53 (1991) 152 [Yád. Fiz. 53 (1991) 240]; B.A. Knihel, Nucl. Phys. B376 (1992) 3: A. Dabelstein and W. Hollik, Z. Phrs. C53 (1992) 507: L. Durand, K. Riesselmann and B.A. Knichl, Phys. Rev. Lett. 72 (1994) 253-1 [E: 74 (1995) 1699]; Phys. Rer. D51 (1995) 5007.

[31] A.L. Kataev, JE'lP Lett. 66 (1997) 327 [Pis'ma Zh. Eksp. Teor. Fiz. 66 (1997) 308].

[32] M. Spira, hep-ph/9810289.

[33] See. eg., H. Fusauka and Y. Koide, Phys. Rev. D57 (1998) 3986.

[34] A computer program library with cross-sections for the Higgs production processes (in the Standard Model and the MSSM) that are relevant for hadron collider Higgs searches is available from http://home.cern.ch/ ${ }^{\sim}$ mspira/proglist.html. 
[35] M. Spira, private communication. Fig. 5 updates the result presented in ref. [32] by including the NLO QCD-corrected cross-section for $h_{\mathrm{SM}} t \bar{t}$ production.

[36] D. Dicus, T. Stelzer, Z. Sullivan and S. Willenbrock, Phys. Rev. D59 (1999) 094016.

[37] H. Georgi, S. Glashow, M. Machacek and D.V. Nanopoulos, Phys. Rev. Lett. 40 (1978) 692

[38] A. Djouadi, M. Spira and P.M. Zerwas, Phys. Lett. B264 (1991) 440; S. Dawson, Nucl. Phys. B359 (1991) 283; D. Graudenz, M. Spira and P.M. Zerwas, Phys. Rev. Lett. 70 (1993) 1372; M. Spira, A. Djouadi, D. Graudenz and P.M. Zerwas, Nucl. Phys. B453 (1995) 17.

[39] M. Krämer, E. Laenen and M. Spira, Nucl. Phys. B511 (1998) 523; C. Balázs and C.-P. Yuan, Phys. Lett. B478 (2000) 192.

[40] V. Ravindran, J. Smith and W.L. Van Neerven, Nucl. Phys. B634 (2002) 247.

[41] R.V. Harlander and W.B. Kilgore, Phys. Rev. Lett. 88 (2002) 201801; C. Anastasiou and K. Melnikov, Nucl. Phys. B646 (2002) 220.

[42] T. Han and S. Willenbrock, Phys. Lett. B273 (1991) 167.

[43] S. Mrenna and C.P. Yuan, Phys. Lett. B416 (1998) 200.

[41] M. Spira, Fortschr. Phys. 46 (1998) 203.

[45] T. Han, G. Valencia and S. Willenbrock, Phys. Rev. Lett. 69 (1992) 3274.

[46] L. Reina and S. Dawson, Phys. Rev. Lett. 87 (2001) 201804; L. Reina, S. Dawson and D. Wackeroth, Phys. Rev. D65 (2002) 053017.

[47] W. Beenakker, S. Dittmaier, M. Kramer, B. Plumper, M. Spira and P.M. Zerwas, Phys. Rev. Lett. 87 (2001) 201805.

[48] R.M. Barnett, H.E. Haber and D.E. Soper, Nucl. Phys. B306 (1988) 697.

[49] D.A. Dicus and S. Willenbrock, Phys. Rev. D39-(1989) 751.

[j0] D. Rainwater, M. Spira and D. Zeppenfeld, hep-ph/0203187.

[51] D. Choudhury, A. Datta and S. Raychaudhuri, hep-ph/9809552; C.S. Huang and S.H. Zhu. Phys. Rev. D60 (1999) 075012.

5.5] J. Campbell, R.K. Ellis, F. Maltoni and S. Willenbrock, hep-ph/0204093. 
[53] E.W. Glover and J.J. van der Bij, Nucl. Phys. B309 (1988) 282.

[54] T. Plehn, M. Spira and P.M. Zerwas, Nucl. Phys. B479 (1996) 46 [E: B531 (1998) 655.

[55] S. Dawson, S. Dittmaier and M. Spira, Phys. Rev. D58 (1998) 1.15012.

[56] A. Djouadi, W. Kilian, M. Mühlleitner and P.M. Zerwas, Eur. Phrss. J. C10 (1999) 45.

[57] V.A. Khoze, A.D. Martin and M.G. Ryskin, Eur. Phys. J. C14 (2000) 525; C23 (2002) 311 B. Cox, J. Forshaw and B. Heinemann, Phys. Lett. B540 (2002) 263; R. Enberg, G. Ingelman. A. Kissavos and N. Timneanu, Phys. Rcr. Lett. 89 (2002) 081801

[58] V.A. Khoze, A.D. Martin and M.G. Ryskin, IPPP/02/44 and DCPT/02/88 [hep-ph/0207313]

[59] A. De Roeck, V.A. Khoze, A.D. Martin, R. Orava and M.G. Ryskin, Eur. Phys, J. C25 (2002) 391.

[60] T. Han, A.S. Turcot and R.-J. Zhang, Phys. Rev. D59 (1999) 093001

[61] A. Stange, W. Marciano and S. Willenbrock, Phys. Rcv. D49 (1994) 1354: D50 (1994) 4491.

[62] M. Carena, J.S. Conway, H.E. Haber, J. Hobbs et al., Report of the Tevatron Higgs Working Group, hep-ph/0010338.

[63] P.C. Bhat, R. Gilmartin and H.B. Prosper, Phys. Rev. D62 (2000) 074022.

[64] C. Nelson, Phys. Rev. D37 (1988) 1220.

[65] M. Dittmar and H. Dreiner, Phys. Rev. D55 (1997) 167; and in The Higgs Puzzle - What can W: Learn from LEP2, $L H C, N L C$, and FMC?, Proceedings of the 1996 Ringberg Workshop, Ringberg Castle, Tegernsee, Germany, 8-13 December, 1996, edited by B.A. Kniehl (World Scientific, Singapore, 1997) pp. 113-121.

[66] A. Belyaev, T. Han and R. Rosenfeld, FSU-HEP-020417 [hep-ph/0204210].

[67] J. Goldstein, C.S. Hill, J. Incandela, S. Parke, D. Rainwater and D. Stuart. Phys. Rev. Lett. 86 (2001) 1694.

[68] J. Conway, K. Desch, J.F. Gunion, S. Mrenna and D. Zeppenfeld, Report of the P1-WG2 subgroup, "The Precision of Higgs Boson Measurements and their Implications," in Proceedings of the APS/DPF/DPB Summer Study on the Future of Particle Physics (Snowmass 2001), edited by R. Davidson and C. Quigg, SNOWMASS-2001-P1WG2 [hep-ph/0203206]. 
[69] S. Dawson, "Higgs boson production rates in hadronic collisions," in Proceedings of the APS/DPF/DPB Summer Study on the Future of Particle Physics (Snowmass 2001), edited by R. Davidson and C. Quigg, SNOWMASS-2001-P124 [hep-ph/0111226].

[70] See e.g., ATLAS Collaboration, ATLAS Detector and Physics Performance: Technical Design Report, Volume 2, ATLAS TDR 15, CERN/LHCC 99-15. The LHC Higgs search is discussed in Chapter 19; D. Costanzo, "Higgs Physics at the Large Hadron Collider," presented at the 36th Rencuntres de Moriond on Electroweak Inleractions and Unified Theories, Les Arcs, France. 10-17 March 2001 [hep-ex/0105033].

[71] K. Lassila-Perini, dissertation, ETH No. 12961 (1998) [CERN-THESIS-99-3]; "Higgs Physics at the LHC," presented at the III International Symposium on LHC Physics and Detectors, Chia, 2527 October 2001, CMS Conference Report CMS CR 2001/018.

[72] M. Dittmar, Pramana 55 (2000) 151; M. Dittmar and A.-S. Nicollerat, "High Mass Higgs Studies using $g g \rightarrow h_{\mathrm{SM}}$ and $q q \rightarrow q q h_{\mathrm{SM}}$ at the LHC;" CMS-NOTF, 2001/036.

[73] T. Trefzger, Fortschr. Phys. 49 (2001) 1147.

[74] J.G. Branson, D. Denegri, I. Hinchliffe, F. Gianotti, F.E. Paige and P. Sphicas, editors |The ATLAS and CMS Collaborations], EPJdirect CN1 (2002) 1.

[i5] D. Cavalli et al., Report of the Higgs working group for the Workshop "Physics at TeV Colliders." Les Houches, France, 21 May-1 June 2001, hep-ph/0203056.

[76] T. Trefzger, "Search for Higgs Bosons at LHC," in Proceedings of the APS/DPF/DPB Summer Study on the Future of Particle Physics (Snowmass 2001), edited by R. Davidson and C. Quigg, SNOWMASS-2001-P111.

[iT] V. Drollinger, Th. Muller and D. Denegri, CMS NOTE 2002/006 [hep-ph/0201249].

[78] D. Zeppenfeld, R. Kinnunen, A. Nikitenko and E. Richter-Was, Phys. Rev. D62 (2000) 013009; D. Zeppenfeld, "Higgs Couplings at the LHC," in Proceedings of the APS/DPF/DPB Summer Study on the Future of Particle Physics (Snowmass 2001), edited by R. Davidson and C. Quigg, SNOWMASS-2001-P123 [hep-ph/0203123].

[79] T. Plehn, D. Rainwater and D. Zeppenfeld, Phys. Rev. Lett. 88 (2002) 051801.

[80] T. Han and B. McElrath, Phys. Lett. B528 (2002) 81.

[81] D. Rainwater and D. Zeppenfeld, Phys. Rev. D60 (1999) 113004 [E: D61, 099901 (1999)]; N. Kauer, T. Plehn, D. Rainwater and D. Zeppenfeld, Phys. Lett. B503 (2001) 113. 
[82] E. Richter-Was and M. Sapinski, Act. Phys. Pol. B30 (1999) 1001: M. Sapinski and D. Cavall, Act. Phys. Pol. B32 (2001) 1317.

[83] D. Green, K. Maeshima, R. Vidal and W. Wu, CMS NOTE 2001/039; D. Green. K. Maeshisna. R. Vidal, W. Wu and S. Kunori, FERMILAB-FN-0705 (2001): V. Drollinger. Th. Müller and D. Denegri, CMS NOTE 2001/054 [hep-ph/0111312].

[84] A. Belyaev and L. Reina. JHEP 0208 (2002) 041.

[85] F. Maltoni, D. Rainwater and S. Willenbrock. Phys. Rev. D66 (2002) 034022.

[86] U. Baur, T. Plehn and D. Rainwater, Phys. Rev. Lett. 89 (2002) 151801.

87] F. Gianotti, M.L. Mangano and T. Virdee conveners, Physics Polential und Expermimentul Chatlenges of the LHC Luminosity Upgrade, CERN-TH/2002-078 [hep-ph/0204087].

88] X. Akasaka et ul., "JLC design study," KEK-REPURT-9'-1.

[89] C. Adolphsen ot ul. [International Study Group Collaboration]. "International study group) progress report on linear collider development," SLAC-R-559 and KEK-REPORT-2000-7 April. 2000).

[90] R. Brinkmann, K. Flottmann, J. Rossbach, P. Schmuser, N. Walker and H. Weise [editons]. "TESLA: The superconducting electron positron linear collider with an integrated X-ray laser laboratory. Technical design report, Part 2: The Accelerator." DESY-01-011. March, 20)011. [http://tesla.desy.de/tdr/].

[91] R.W. Assmann et al. [The CLIC Study Team], "A 3 TeV e" thear collider based on (Lic: technology," edited by G. Guignard, SLAC-REPRINT-2000-096 and CERN-2000-008.

[92] R.D. Heuer, D.J. Miller. F. Richard and P.M. Zerwas [editors], "TESLA: The superom ducting electron positron linear collider with an integrated X-ray laser laboratury. 'Technical design report, Part 3: Physics at an $e^{+} e^{-}$Linear Collider." DESY-01-011 (March. 2001). http://tesla.desy.de/tdr/ [hep-ph/0106315].

[93] T. Abe et al. [American Linear Collider Working Group Collaboration?, SLAC-R-57) (2001) |hepex/0106055-58].

[94] ACFA Linear Collider Working Group, K. Abe et al., KEK-REPORT-2001-1L (2001) the tph/0109166]. 
[95] J. Ellis, M.K. Gaillard, D.V. Nanopoulos, Nucl. Phys. B106 (1976) 292; B.L. Ioffe, V.A. Khoze. Sov. J. Nucl. Phys. 9 (1978) 50.

[96] D.R.T. Jones and S. Petcov, Phys. Lett. B84 (1979) 440; R.N. Cahn and S. Dawson, Phys. Lett. B136 (1984) 196; G.L. Kane, W.W. Repko and W.B. Rolnick, Phys. Lett. B148 (1984) 367; G. Altarelli, B. Mele and F. Pitolli. Nucl. Phys. B287 (1987) 205; W. Kilian, M. Krämer and P.M. Zerwas, Phys. Lett. B373 (1996) 135.

[97] B.A. Kniehl, Z. Phys. C55 (1992) 605.

[98] J. Fleischer and F. Jegerlehner, Nucl. Phys. B216 (1983) 469; A. Demer, J. Kublbeck, R. Merlig and M. Bohm, Z. Phys. C56 (1992) 261.

[99] B.A. Kniehl, Int. J. Mod. Phys. A17 (2002) 1457.

100] D. Choudhury, T.M. Tait and C.E.M. Wagner, Phys. Rev. D65 (2002) 115007.

[101 K.J. Gaemers and G.J. Gounaris, Phys. Lett. 77B (1978) 379; A. Djouadi, J. Kalinowski and P. M. Zerwas, Z. Phys. C54 (1992) 255: B.A. Kniehl, F. Madricardo and M. Steinhauser, Phys. Rev. D66 (2002) 054016.

[102] S. Dittmaier, M. Kramer, Y. Liao, M. Spira and P.M. Zerwas, Phys. Lett. B441 (1998) 383; B478 (2000) 247; S. Dawson and L. Reina, Phys. Rev. D59 (1999) 054012.

[103] D.J. Miller, S.Y. Choi, B. Eberle, M. Mühlleitner and P.M. Zerwas, Phys. Lett. B505 (2001) 149; P. Garcia, W. Lohmann and A. Raspereza, LC-Note LC-PHSM-2001-054.

[104] V.D. Barger, K. Cheung, A. Djouadi, B.A. Kniehl and P.M. Zerwas, Phys. Rev. D49 (1994) 79.

105.) D. Chang, W.-Y. Keung and I. Phillips, Phys. Rev. D48 (1993) 3225; A. Soni and R.M. Xu, Phys. Rev. D48 (1993) 5259; A. Skjold and P. Osland, Phys. Lett. B329 (1994) 305: Nucl. Phys. B453 (1995) 3; B. Grzadkowski and J.F. Gunion, Phys. Let.t. B350 (1995) 218; T. Han and J. Jiang. Phys. Rev. D63 (2001) 096007.

[106] J.F. Gunion, B. Grzadkowski and X.-G. He, Phys. Rev. Lett. 77 (1996) 5172: K. Hagiwara. S. Ishihara, J. Kamoshita and B.A. Kniehl, Eur. Phys. J. C14 (2000) 457.

[107] K. Desch and M. Battaglia, in Physics and experiments with future linear $e^{+} e^{-}$colliders, Proceedings of the 5th International Linear Collider Workshop, Batavia, IL, USA, 2000, edited by A. Para and H.E. Fisk (American Institute of Physics, New York, 2001), pp. 312-316: M. Battaglia and K. Desch, in ibid., pp. 163182 [hep-ph/0101]65]. 
[108] E. Boos, J.C. Brient, D.W. Reid, H.J. Schreiber and R. Shanidze, Eur. Phys. J. C19 (2001) 45.j.

[109] M. Battaglia and A. De Roeck, "Determination of the muon Yukawa coupling at high energy " " linear colliders," in Proceedings of the APS/DPF/DPB Summer Study on the Future of Partich" Physics (Snowmass 2001), edited by R. Davidson and C. Quigg SNOWMASS-2001-E3066 [hep$\mathrm{ph} / 0111307]$.

[110] C.T. Potter, J.E. Brau and M. Iwasaki. "Standard Model Higgs Bosin Branching Ratio Moasme. monts at a Linear Collider:" in Proceedings of the APS/DPF/DPB Sumner Study on the Future of Particle Physics (Snowmass 2001), edited by R. Davidson and C. (Quigg, SNOWMASS-2001-1'118.

[111] J.F. Gunion, B. Grzadkowski and X.G. He, Phys. Re'v. Leth. 77 (1996) 5172, A. Juste and G. Merino, hep-ph/9910301: H. Baer, S. Dawson and L. Reina. Phys Rur. D61 (2000) 01300?.

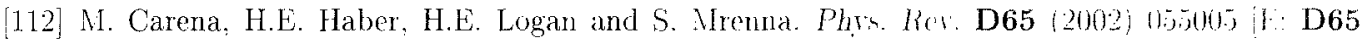
(2002) 099902].

[113] A. Djouadi, H.E. Haber and P.M. Zerwas, Phys. Lett. B375 (1996) 203; F. Bourijema anu! E. Chopin, Z. Phys. C73 (1996) 85; J. Kamoshita. Y. Okada. N. Tanaka and I. Watanaties. hep-ph/9602224; D.J. Miller and S. Moretti. Eur. Phys. I. C13 (2000) 459.

[114] A. Djouadi, W. Kilian, M. Mühlleitner and P.V. Zerwas, Fim Phys I. C10 (1999) 27: C (Adstanier. P. Gay, P. Lutz and J. Orloff, LC Note LC-PHSM-2000-061 [hep-ex/0101028].

[115] M. Battaglia, E. Boos and W. Yao, "Studying the Higgs Potential at the, , Lituar Colliter." in Proceedings of the APS/DPF/DPB Summer Study on the Fulure of Particle Physics (Sromwants2001). edited by R. Davidson and C. Quigg, SNOWMASS-2001-E3016 [hep-ph/0111276].

[116] K. Desch and N. Meyer. LC Note LC-PHSM-2001-25.

[11i] P.F. Derwent et al, "Linear collider physics," a report prepared for Fermilab Directorate hepex/0107044].

[118] J. Alcaraz and E. Ruiz Morales, Phys. Rev. Lett. 86 (2001) 3726.

[119] I.F. Ginzburg, G.L. Kotkin, S.L. Panfil, V.G. Serbo and V.I. Telnov, Nucl. Inst. Meth. A219 (1984) 5 .

[120] E. Boos et al., Nucl. Inst. Meth. A472 (2001) 100.

[121] D.L. Borden, D.A. Bauer and D.O. Caldwell, Phys. Rev. D48 (1993) 1018: J.F. Gunion and H.F. Haber, Phys Rev. D48 (1993) 5109. 
122] S. Söldner-Rembold and G. Jikia, Nucl. Inst. Meth. A472 (2001) 133; M. Melles, Nucl. Inst. Meth. A472 (2001) 128.

[123] D.M. Asner, J.B. Gronberg and J.F. Gunion, hep-ph/0110320.

[124] M.M. Velasco et al., "Photon-Photon and Electron-Photon Colliders with Energies Below a leV," in Proceedings of the APS/DPF/DPB Summer Study on the Future of Particle Physics (Snowmass 2001), edited by R. Davidson and C. Quigg, SNOWMASS-2001-F3005 [hep-ex/0111055].

[125] D.L. Borden, V.A. Khoze, W.J. Stirling and J. Ohnemus, Phys. Rev. D50 (1994) 4499; V.S. Fadin, V.A. Khoze and A.D. Martin, Phys. Rev. D56 (1997) 484; M. Melles, W.J. Stirling and V.A. Khoze, Phys. Rev. D61 (2000) 054015.

[126] L.E. Ibáñez and G.G. Ross, Phys. Lett. B110 (1982) 215; L.E. Ibáñez, Phys. Lett. B118 (1982) 73; J. Ellis, D.V. Nanopoulos and K. Tamvakis, Phys. Lett. B121 (1983) 123; L. Alvarez-Gaumé, J. Polchinski and M.B. Wise, Nucl. Phys. B221 (1983) 495.

[127] S. Dimopoulos and H. Georgi, Nucl. Phys. B193 (1981) 150; K. Harada and N. Sakai, Prog. Theor. Phys. 67 (1982) 1877; K. Inoue, A. Kakuto, H. Komatsu and S. Takeshita, Prog. Theor. Phys. 67 (1982) 1889; L. Girardello and M.T. Grisaru, Nucl. Phys. B194 (1982) 65; L.J. Hall and L. Randall, Phys. Rev. Lett. 65 (1990) 2939; I. Jack and D.R.T. Jones, Phys. Lett. B457 (1999) 101.

[128] S. Dimopoulos and D.W. Sutter, Nucl. Phys. B452 (1995) 496; D.W. Sutter, Stanford Ph. D. thesis, hep-ph/9704390; H.E. Haber, Nucl. Phys. B (Proc. Suppl.) 62A-C (1998) 469.

[129] For a review of the two-Higgs-doublet model (both non-supersymmetric and supersymmetric), see Chapter 4 of ref. [2].

[130] H.E. Haber and Y. Nir, Phys. Lett. B306 (1993) 327; J.F. Gunion and H.E. Haber, SCIPP-02/10 and UCD-2002-10 [hep-ph/0207010].

[131] H.A. Weldon, Phys. Lett. B146 (1984) 59; Phys. Rev. D30 (1984) 1547.

[132] J.F. Gunion, H.E. Haber and J. Wudka, Phys. Rev. D43 (1991) 904; J.F. Gunion, B. Grzadkowski, H.E. Haber and J. Kalinowski, Phys. Rev. Lett. 79 (1997) 982; B. Grzadkowski, J.F. Gunion and J. Kalinowski, Phys. Rev. D60 (1999) 075011.

[133] L.J. Hall and M.B. Wise, Nucl. Phys. B187 (1981) 397.

[134] Y. Okada, M. Yamaguchi and T. Yanagida, Prog. Theor. Phys. 85 (1991) 1; J. Ellis, G. Ridolfi and F. Zwirner, Phys. Lett. B257 (1991) 83. 
[135] S.P. Li and M. Sher, Phys. Lett. B140 (1984) 339; R. Barbieri and M. Frigeni, Phys. Lett. B258 (1991) 395; M. Drees and M.M. Nojiri, Phys. Rev. D45 (1992) 2482; J.A. Casas, J.R. Espinosa. M. Quirós and A. Riotto, Nucl. Phys. B436 (1995) 3 [E: B439 (1995) 466].

[136] J. Ellis, G. Ridolfi and F. Zwirner, Phys. Lett. B262 (1991) 477: A. Brignolo. J. Ellis, G. Ridolti and F. Zwirner, Phys. Lett. B271 (1991) 123 [E: B273 (1991) 550].

[137] R.-J. Zhang, Phys. I.ett. B447 (1999) 89

[138] J.R. Espinosa and R.-J. Zhang, JHEP 0003 (2000) 026.

139] J.R. Espinosa and R.-J. Zhang. Nurl. Phys. B586 (2000) 3; A. Brignole, (i. Degrassi. P. Slavith and F. Zwirner, Nucl. Phys. B631 (2002) 195.

[140] A. Brignole, G. Degrassi, P. Slavich and F. Zwirner, Nucl. Phys. B643 (2002) 79.

111] H.E. Haber and R. Hempfling, Phys, Rer Lett. 66 (1991) 1815.

[142] J.F. Gunion and A. Turski, Phys. Rev. D39 (1989) 2701; Phys. Rev. D40 (1989) 2333; M.S. Berger, Phys. Rev. D41 (1990) 225; A. Brignole, Phys. Lett. B277 (1992) 313; B281 (1992) 284; M.A. Díaz and H.E. Haber, Phys. Rev. D45 (1992) 4246.

[143] P.H. Chankowski. S. Pokorski and J. Rosiek. Phy's. Lot. B274 (1992) 191: Nucl. Phws. B423 (1994) 437; A. Yamada. Phys. Lett. B263 (1991) 233: Z. Phrs. C61 (1994) 247; A. Dabelstein. Z. Phys. C67 (1995) 495.

[1.44] D.M. Pierce. J.A. Bagger. K. Matchev, and R. Zhang, Niuel. Phrs. B491 (1997) :

145] R. Hempfling and A.H. Hoang. Phys. Lett. B331 (1994) 99.

[146] S. Heinemeyer, W. Hollik and G. Weiglein, Phrs. Rev. D58 (1998) 091701: Phys. Rev. Lett 440 (1998) 296; Eur. Phys. T. C9 (1999) 343.

[147] R. Barbieri, M. Frigeni and F. Caravaglios, Phys. Lett. B258 (1991) 167: Y. Okada. I Kamaguchi and T. Yanagida, Phys. Lett. B262 (1991) 54; J.R. Espinosa and M. Quirós, Phys. Lett. B266 (1991) 389; D.M. Pierce, A. Papadopoulos and S. Johnson, Phws. Rev. Lett. 68 (19992) 3678; K. Sasaki, M. Carena and C.E.M. Wagner, Nucl. Phys. B381 (1992) 66; R. Hempfling, in Phenomenological Aspects of Supersymmetry, edited by W. Hollik, R. Rückl and J. Wess (SpringerVerlag, Berlin. 1992) pp. 260-279; J. Kodaira, Y. Yasui and K. Sasaki, Phys. Rev. D50 (1994) 7035 .

[148] H.E. Haber and R. Hempfling, Phys. Rev. D48 (1993) 4280. 
[149] M. Carena, J.R. Espinosa, M. Quirós and C.E.M. Wagner, Phys. Lett. B355 (1995) 209; M. Carena, M. Quirós and C.E.M. Wagner, Nucl, Phys. B461 (1996) 407.

[150] H.E. Haber, R. Hempfling and A.H. Hoang, Z. Phys. C75 (1997) 539.

[151] M. Carena, H.E. Haber, S. Heinemeyer, W. Hollik, C.E.M. Wagner and G. Weiglein, Nucl. Phys. B580 (2000) 29.

[152] M. Carena, S. Mrenna and C.E.M. Wagner, Phys. Rev. D60 (1999) 075010.

[153] M. Carena, J. Ellis, A. Pilaftsis and C.E.M. Wagner, Nucl. Phys. B586 (2000) 92.

[154] S. Heinemeyer, W. Hollik and G. Weiglein, Phys. Lett. B455 (1999) 179.

[155] J.R. Espinosa and I. Navarro, Nucl. Phys. B615 (2001) 82; G. Degrassi, P. Slavich and F. Zwirner, Nucl. Phys. B611 (2001) 403.

[156] M. Carena, S. Heinemeyer, C.E.M. Wagner and G. Weiglein, CERN-TH-99-374 [hcp-ph/9912223].

[157] S. Heinemeyer, W. Hollik and G. Weiglein, Comp. Phys. Commun. 124 (2000) 76; CERN-TH2000 055 [hep ph/0002213] describe the programs FeynHiggs and FeynHiggsFast, respectively. The program subpole of M. Carena, M. Quirós and C.E.M. Wagner now includes the leading two-loop non-logarithmic threshold corrections. The program hmsusytev, based on ref. [150], now includes the one-loop supersymmetric threshold corrections to the relation between the third generation Higgs Yukawa couplings and quark masses.

[158] D. Comelli and J.R. Espinosa, Phys. Lett. B388 (1996) 793.

[159] J.M. Moreno, D.H. Oaknin and M. Quirós, Nucl. Phys. B483 (1997) 267.

[160] M. Carena, S. Mrenna and C.E.M. Wagner, Phys. Rev. D62 (2000) 055008.

[161] ALEPH, DELPHI, L3 and OPAL Collaborations, The LEP working group for Higgs boson searches, LHWG Note 2001-04 (July 2001) [hep-ex/0107030].

[162] S. Heinemeyer, W. Hollik and G. Weiglein, JHEP 0006 (2000) 009.

[163] ALEPH, DELPHI, L3 and OPAL Collaborations, The LEP working group for Higgs boson searches, LHWG Note 2001-05 (July 2001) [hep-ex/0107031].

[164] A. Pilaftsis, Phys. Rev. D58 (1998) 096010; Phys. Lett. B435 (1998) 88; K.S. Babu, C.F. Kolda, J. March-Russell and F. Wilczek, Phys. Rev. D59 (1999) 016004; D.A. Demir, Phys. Rev. D60 (1999) 055006; M. Carena, J. Ellis, A. Pilaftsis and C.E.M. Wagner, Phys. Lett. B495 (2000) 155; M. Carena, J.R. Ellis, A. Pilaftsis, C.E.M. Wagner, Nucl Phys. B625 (2002) 345. 
[165] G.L. Kane and L.-T. Wang, Phys. Lett. B488 (2000) 383; S.Y. Choi, M. Drees and J.S. Lee, Phys. Lett. B481 (2000) 57; S.Y. Choi and J.S. Lee, Phys. Rev. D61 (2000) 015003; S.Y. Choi, K. Hagiwara and J.S. Lee, Phys. Rev. D64 (2001) 032004; Phys. Lett. B529 (2002) 212: T. Ibrahim and P. Nath, Phys. Rev. D63 (2001) 035009; T. Ibrahim, Phys. Rev. D64 (2001) 035009: S. Heinemeyer, Eur. Phys. J. C22 (2001) 521; S.W. Ham, S.K. Oh. E.J. Yoo. C.M. Kim and D. Son. hep-ph/0205244.

[166] A. Pilaftsis and C.E.M. Wayner, Nucl. Plyys. B553 (1999) 3.

[167] S. Dimopoulos and S. Thomas, Nucl. Phys. B465 (1996) 23: S. Thomas. Int. J. Mod. Phys. A13 (1998) 2307.

[168] J.F. Gunion, H.E. Haber and J. Kalinowski, in preparation.

[169] W. Loinaz and J.D. Wells, Phys, Lett. B445 (1998) 178: K.S. Babu and C.F. Kolda, Phys. Lett. B451 (1999) 77 .

[170] C. Balázs, J.L. Diaz-Cruz, H,-J. He, T. Tait and C.P. Yuan. Phys. Rev. D59 (1999) 055016.

[171] A. Dabelstein, Nucl. Phys. B456 (1995) 25; F. Borzumati, G.R. Farrar. N. Polonsky and S. Thomas, Nucl. Phys. B555 (1999) 53; H. Eberl, K. Hidaka. S. Kraml, W. Majerotto anl Y. Yamada, Phys. Rev. D62 (2000) 055006.

[172] J.A. Coarasa, R.A. Jiménez and J. Solà, Phys. Lett. B389 (1996) 312; R.A. Jiménez and J. Solà. Phys. Lett. B389 (1996) 53; A. Bartl, H. Eberl, K. Hikasa, T. Kon. W. Majerotto and Y. Yamada. Phys. Lett. B378 (1996) 167.

[173] S. Heinemeyer, W. Hollik and G. Weiglein, Eur. Phys. J. C16 (2000) 139.

[174] H.F. Haber, M.J. Herrero, H.E. Logan, S. Peñaranda, S. Rigolin and D. Temes, Phys Rev. D63 (2001) 055004 .

[175] L. Hall, R. Rattazzi and U. Sarid, Phys. Rev. D50 (1994) 7048; R. Ilempfling, Phys. Rev. D49 (1994) 6168 .

[176] M. Carena, M. Olechowski, S. Pokorski and C.E.M. Wagner, Nuci. Fhyss. B426 (1994) 269.

[177] M. Carena, D. Garcia, U. Nierste and C.E.M. Wagner, Phys. Lett. B499 (2001) 141.

[178] E. Christova, H. Eberl, W. Majerotto and S. Kraml, Nucl. Phys. B639 (2002) 263 [E: B647 (2002) 359]. 
[179] E. Boos, A. Djouadi, M. Mühlleitner and A. Vologdin, Phys. Rev. D66 (2002) 055004.

[180] S. Mrenna, private communication.

181] A. Djouadi, J. Kalinowski and P.M. Zerwas, Z. Phys. C70 (1996) 435; S. Moretti and W.J. Stirling, Phys. Lett. B347 (1995) 291 [E: B366 (1996) 451].

[182] A. Mendez and A. Pomarol, Phys. Lett. B252 (1990) 461; C.-S. Li, and R.J. Oakes, Phys. Rev. D43 (1991) 855; M. Drees and D.P. Roy, Phys. Lett. B269 (1991) 155; A. Djouladi and P. Gambino, Phys. Rev. D51 (1995) 218.

[183] X.-J. Bi, Y.-B. Dai and X.-Y. Qi, Phys. Rev. D61 (2000) 015002.

[181] A. Djouadi, J. Kalinowski, P.M. Zerwas, 7. Phys. C57 (1993) 569; H. Baer, M. Bisset. D. Dicus, C. Kao and X. Tata, Phys. Rev. D47 (1993) 1062; A. Djouadi, P. Janot, J. Kalinowski and P.M. Zerwas, Phys. Lett. B376 (1996) 220; A. Djouadi, J. Kalinowski, P. Ohmann and P.M. Zerwas, Z. Phys. C74 (1997) 93.

[185] J.F. Gunion, Phys. Rev. Lett. 72 (1994) 199; D. Choudhury and D.P. Roy, Phys. Lett. B322 (1994) 368; O.J. Eboli and D. Zeppenfeld, Phys. Lett. B495 (2000) 147; B.P. Kersevan, M. Malawski and E. Richter-Was, hep-ph/0207014.

[186] J.F. Gunion and H.E. Haber, Nucl. Phys. B278 (1986) 449 [E: B402 (1993) 567].

[187] S. Dawson, A. Djouadi and M. Spira, Phys. Rev. Lett. 77 (1996) 16; A. Djouadi, Phys. Lett. B435 (1998) 101.

[188] A. Djouadi and M. Spira, Phys. Rev. D62 (2000) 014004.

[189] A. Djouadi, M. Spira, D. Graudenz and P.M. Zerwas, Phys. Lett. B318 (1993) 347.

[190] R.V. Harlander and W.B. Kilgore, JHEP 0210 (2002) 017; C. Anastasiou and K. Melnikov, SLAC-PUB-9373 [hep-ph/0208115].

[191] C. Balázs, H.-J. He and C.P. Yuan, Phys. Rev. D60 (1999) 114001.

[192] R.M. Godbole and D.P. Roy, Phys. Rev. D43 (1991) 3610; M. Guchait and P. Roy, Phys. Rov. D55 (1997) 7263; F.M. Borzumati and N. Polonsky, in $e^{+} e^{-}$Collisions at TeV Energies: the Physics Potential, Proceedings edited by P.M. Zerwas, DESY-96-123D, p. 41 [hep-ph/9602433]; F.M. Borzumati and A. Djouadi, hep-ph/9806301. E. Accomando et al., Phys. Reports 299 (1998) 1; J.A. Coarasa, J. Guasch, J. Solà and W. Hollik, Phys. Lett. B442 (1998) 326; J. Guasch and J. Solà, Phys. Lett. B416 (1998) 353. 
[193] J.A. Coarasa, D. Garcia, J. Guasch, R.A. Jimenez and J. Solà: Eur. Phys. J. C2 (1998) 373.

[194] C.S. Li and 'I.C. Yuan, Phys. Rev. D42 (1990) 3088 [E: D47 (1993) 2156]; A. Czarnecki and S. Davidson, Phys. Rev. D47 (1993) 3063; C.S. Li, Y.-S. Wei and J.-M. Yang, Phrs. Lett. B285 (1992) 137.

[195] J. Guasch, R.A. Jiménez and J. Solà, Phys. Lett. B360 (1995) 47.

[196] M. Carena, D. Garcia, U. Nierste and C. Wagner, Nucl. Phys. B577(2000) 88.

[197] R. Bonciani, S. Catani, M.L. Mangano and I. Nason, Nucl. Phys. B529 (1998) 424.

[198] M. Guchait and S. Moretti, JHEP 0201 (2002) 001.

[199] K.A. Assamagan et al., in ref. [87], pp. 85-120.

[200] F. Olness and W.-K. Tung, Nucl. Phys. B308 (1988) 813.

[201] F. Borzumati, J.-L. Kneur and N. Polonsky, Phys. Rev. D60 (1999) 115011.

[202] A. Belyaev. D. Garcia, J. Guasch and J. Solà, JHEP 0206 (2002) 059.

[203] L.G. Jin, C.S. Li, R.J. Oakes and S.H. Zhu, Eur. Phys. J. C14 (2000) 91; Phys. Rev. D62 (2000) 053008; A. Belyaev, D. Garcia, J. Guasch and J. Solà, Phys. Rev. D65 (2002) 031701; G. Gao. G. Lu, Z. Xiong and J.M. Yang, Phys. Rev. D66 (2002) 015007.

[204] S.-H. Zhu, hep-ph/0112109; T. Plehn, MADPH-02-1275 [hep-ph/0206121].

[205] A.A. Barrientos Bendezú and B.A. Kniehl, Phys. Rev. D59 (1999) 015009; D61 (2000) 097701; D63 (2001) 015009 .

[206] O. Brein, W. Hollik and S. Kanemura, Phys. Rev. D63 (2001) 095001.

[207] A.A. Barrientos Bendezú and B.A. Kniehl, Nucl. Phys. B568 (2000) 305.

[208] A. Krause, T. Plehn, M. Spira and P.M. Zerwas, Nucl. Phys. B519 (1998) 85.

[209] O. Brein and W. Hollik, Eur. Phys. J. C13 (2000) 175.

[210] A. Belyaev, M. Drees, O.J. Eboli, J.K. Mizukoshi and S.F. Novaes, Phys. Rev. D60 (1999) 075018:

A. Belyaev, M. Drees and J. K. Mizukoshi. Eur. Phys. J. C17 (2000) 337. 
[211] A.A. Barrientos Bendezú and B.A. Kniehl, Phys. Rev. D64 (2001) 035006; R. Lafaye, D.J. Miller, M. Mühlleitner and S. Moretti, DESY-99-192 [hep-ph/0002238], contributed to Workshop on Physics at TeV Colliders, Les Houches, France, 7-18 Jun 1999.

[212] K.T. Matchev and S. Thomas, Phys. Rev. D62 (2000) 077702.

[213] I. Hinchliffe, F.E. Paige, M.D. Shapiro, J. Soderqvist and W. Yao, Phys. Rev. D55 (1997) 5520; S. Abdullin et al. [CMS Collaboration], J. Phys. G28 (2002) 469.

[214] M. Carena, S. Heinemeyer, C.E.M. Wagner and G. Weiglein, hep-ph/0202167.

[215] D. Rainwater, D. Zeppenfeld and K. Hagiwara, Phys. Rev. D59 (1999) 014037; T. Plehn, D. Rainwater and D. Zeppenfeld, Phys. Rev. D61 (2000) 093005.

[216] J. Dai, J.F. Gunion and R. Vega, Phys. Lett. B371 (1996) 71; B387 (1996) 801; J.L. Diaz-Cruz, H.J. He, T. Tait and C.P. Yuan, Phys. Rev. Lett. 80 (1998) 4641.

[217] H. Baer, B. Harris and X. Tata, Phys. Rev. D59 (1999) 015003.

[218] D. Denegri et al., "Summary of the CMS discovery potential for the MSSM SUSY Higgses," CMS NOTE 2001/032 [hep-ph/0112045].

[219] D. Cavalli, R. Kinnunen, G. Negri, A. Nikitenko and J. Thomas, in ref. [75], pp. 67-79.

[220] S. Moretti and D.P. Roy, Phys. Lett. B545 (2002) 329.

[221] K.A. Assamagan, Acta Phys. Polon. B31 (2000) 863; B31 (2000) 881; K.A. Assamagan and Y. Coadou, Acta Phys. Polon. B33 (2002) 707; K.A. Assamagan, Y. Coadou and A. Deandrea, EPJdirect C9 (2002) 1.

[222] Fig. 27(b) was provided by E. Richter-Was and F. Gianotti on behalf of the ATLAS collaboration.

[223] M. Carena, J. Ellis, S. Mrenna, A. Pilaftsis and C.E.M. Wagner, in preparation.

2224] J.F. Gunion, L. Roszkowski, A. Turski, H.E. Haber, G. Gamberini, B. Kayser, S.F. Novaes, F.I. Olness and J. Wudka, Phys. Rev. D38 (1988) 3444.

[225] A. Djouadi, Montpellier preprint PM-02-13 [hep-ph/0205248].

[226] S.H. Zhu, hep-ph/9901221; S. Kanemura, Eur. Phys. J. C17 (2000) 473; A. Arhrib, M. Capdequi Peyranère, W. Hollik and G. Moultaka, Nucl. Phys. B581 (2000) 34.

[227] H.E. Logan and S. Su, Phys. Rev. D66 (2002) 035001. 
228 A. Gutierrez-Rodriguez and O.A. Sampayo, Phys. Rev. D62 (2000) 055004; A. Gutiere\%Rodriguez, M.A. Hernandez-Ruiz and O.A. Sampayo, J. Phys. Soc. Jap. 70 (2001) 2300): S. Moretti, EPJdirect C15 (2002) 1.

[229] S. Kanemura. S. Moretti and K. Odagiri, JHEP 0102 (2001) 01).

[230] J. Guasch, W. Hollik and S. Penaranda, Phvs. Lett. B515 (2001) 367.

[231] M. Mühlleitner. M. Kräner. M. Spira and P.N. Zerwass, Phys. Latt. B508 (200!) 311

[232] See. e.g., U. Ellwanger, M. Rausch do Traubenberg. and C.A. Savov. Vucl. Phws. B492 (1997) 21; L. Ellwanger and C. Hugonie. Eur. Phys. I. C25 (2002) 297, and references therein.

"233] J. Kamoshita, Y. Okada and M. Tanaka. Phys Lett. B328 (1994) fĩ: S.F. King and P.L. Whitte. Phys. Rev. D53 (1996) 4049.

[234] J.R. Espinosa and .J.F. Gunion, Phvs. Rev. Lett. 82 (1999) $108 \%$

235] [. Ellwanger, J.F. Gunion and C. Hugonie, hep-ph/0111179: J.F. Giunion, H.E. Haber and T. Muroi, in New Directions for High Energy Physics, Proceedings of the 1996 DPF/DPB Summer Study on High Energy Physies, Snowmass 96, edited by D.G. Cassel, L.T. Gennari and R.H. Siemann (Stanford Linear Accelerator Center, Stanford, CA, 1997) pp. $598-602$.

[236] J. Gunion, T. Han. I. Jiang. S. Mrenna and A. Sopczak, "Determination of $\tan ; \beta$ at at Future $c^{\dagger}$ " Linear Collider," in Proceedings of the APS/DPF/DPB Summer Study on the Futurt of Particle. Physics (Snowmass 2001), edited by R. Daridson and C. Quigg. SNOWMASS-2001-P120 [her]$\mathrm{ph} / 0112334]$.

[237] A. Freitas and D. Störkinger, Phys. Rev. D66 (2002) 095014.

[238] B. Grzadkowski and J.F. Gunion, Phys. Lett. B294 (1992) 361; J.F. Gunion and J.G. helly. Phys. Lett. B333 (1994) 110; M. Kramer, J. H. Kuhn. M.L. Stong and P.M. Zerwas, Z. Phys. C64 (1994) 21.

[239] S.Y. Choi and J.S. Lee, Phys. Rev. D62 (2000) 036005; E. Asakawa, S.Y. Choi, K. Hagiwara and J. S. Lee, Phys. Rev. D62 (2000) 115005; S. Bae, B. Chung and P. Ko, KAIST-TH 2002/09 [hep-ph/0205212].

[240] M. Mühlleitner, DESY-THESIS-2000-033 [hep-ph/000812T]; F. Boudjema and A. Semenow. Phys. Rev. D66 (2002) 095007

[241] P. Osland and P.N. Pandita, Phys. Rev. D59 (1999) 055013. 